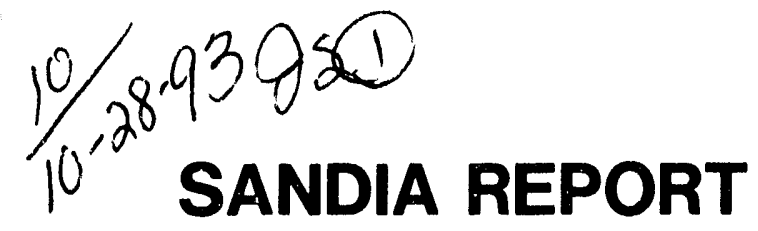

SAND93-1287 $\cdot$ UC -704

Unlimited Release

Printed September 1993

\title{
Continuum Representations of Cellular Solids
}

M. K. Neilsen

Prepared by

Sandia National Laboratories

Albuquerque, New Mexico 87185 and Livermore, Californla 94550

for the United States Department of Energy

under Contract DE-AC04-76DP00789 
Issued by Sandia National Laboratories, operated for the United States Department of Energy by Sandia Corporation.

NOTICE: This report was prepared as an account of work sponsored by an agency of the United States Government. Neither the United States Government nor any agency thereof, nor any of their employees, nor any of their contractors, subcontractors, or their employees, makes any warranty, express or implied, or assumes any legal liability or responsibility for the accuracy, completeness, or usefulness of any information, apparatus, product, or process disclosed, or represents that its use would not infringe privately owned rights. Reference herein to any specific commercial product, process, or service by trade name, trademark, manufacturer, or otherwise, does not necessarily constitute or imply its endorsement, recommendation, or favoring by the United States Government, any agency thereof or any of their contractors or subcontractors. The views and opinions expressed herein do not necessarily state or reflect those of the United States Government, any agency thereof or any of their contractors.

Printed in the United States of America. This report has been reproduced directly from the best available copy.

Available to DOE and DOE contractors from

Office of Scientific and Technical Information

PO Box 62

Oak Ridge, TN 37831

Prices available from (615) 576-8401, FTS 626-8401

Available to the public from

National Technical Information Service

US Department of Commerce

5285 Port Royal Rd

Springfield, VA 22161

NTIS price codes

Printed copy: A06

Microfiche copy: A01 


\title{
Continuum Representations of Cellular Solids*
}

\author{
M. K. Neilsen \\ Engineering Mechanics and Material Modeling \\ Sandia National Laboratories \\ Albuquerque, New Mexico 87185
}

\begin{abstract}
Cellular materials consist of interconnected struts or plates which form cells. The struts or plates are constructed from a variety of metals, polymers, ceramics and wood products. Cellular materials are often used in impact limiters for shipping containers to protect the contents from accidental impact events. These materials exhibit a variety of complex behavior when subjected to crushing loads. This research focuses on the development of continuum representations of cellular solids that can be used in the finite element analysis of shipping container accidents. A significant portion of this work is the development of a new methodology to relate localized deformations to appropriate constitutive descriptions. 'This methodology provides the insight needed to select constitutive descriptions for cellular solids that capture the localized deformations that are observed experimentally. Constitutive relations are developed for two different cellular materials, aluminum honeycomb and polyurethane foam. These constitutive relations are based on plasticity and continuum damage theories. Plasticity is used to describe the permanent deformation exhibited by both aluminum honeycomb and polyurethane foam. Continuum damage is needed to capture the change in elastic parameters due to cracking of the polyurethane cell wall materials. The new constitutive description of polyurethane foam is implemented in both static and dynamic finite element codes, and analytical and numerical predictions are compared with available experimental data.
\end{abstract}

* This report was submitted in partial fulfilment of the requirements for the author's Ph.D. in Engineering from the University of New Mexico under Sandia National laboratories' In-House Dissertation Program. 


\section{Acknowledgements}

I an most grateful to Professor H.L. Schreyer for stimulating discussions and for his guidance and encouragement while serving as my committee chairman. l'm also very appreciative of the discussions with and the encouragement provided by other members of my dissertation committee, Professors R.D. Krieg, A.K. Maji, S. Steinberg, D.Y. Tzou, and W.E. Warren, and my many colleagues at Sandia National Laboratories and the University of New Mexico. Experimental data provided by Professor A.K. Maji and Sunil Donald were very helpful. The financial support provided by Sandia National Laboratories which enabled devotion to this dissertation is gratefully acknowledged. Finally, this dissertation is dedicated to my wife, Susanne, whose patience, understanding and encouragement contributed significantly to the completion of this work. 


\section{Contents}

1. INTRODUCTION . . . . . . . . . . . . . . . . . . . 11

2. CELLULAR MATERIALS . . . . . . . . . . . . . . . . 15

2.1 Gencral Behavior . . . . . . . . . . . . . . . . . . . . 1.5

2.2 Experimental Data . . . . . . . . . . . . . . . . . . . 16

2.2.1 Aluminum Honeycomb . . . . . . . . . . . . . . . . 16

2.2.2 Polyurethanc Foam . . . . . . . . . . . . . . . . . 19

2.3 Existing Continum Representations . . . . . . . . . . . . . . . . . . . 20

3. CONTINUUM CONSTITUTIVE THEORIES . . . . . . . . . 23

3.1 Elasticity . . . . . . . . . . . . . . . . . . . . 23

3.2 Plasticity . . . . . . . . . . . . . . . . . . . . . 24

3.2 .1 Drucker-Prager . . . . . . . . . . . . . . . . . . 25

3.2 .2 voll Mises . . . . . . . . . . . . . . . . . . . . . 26

3.2 .3 Mohr-Coulomb . . . . . . . . . . . . . . . . . . 27

3.2 .4 Principal Stress . . . . . . . . . . . . . . . . . . 28

3.3 Continuum Damage . . . . . . . . . . . . . . . . . . . . . 29

3.3 .1 Simple Scalar Damage . . . . . . . . . . . . . . . . 30

3.3.2 von Mises 1)amage . . . . . . . . . . . . . . . . . 31

3.3.3 Principal Stress Damage . . . . . . . . . . . . . . . 31

3.3.4 Principal Strain Damage . . . . . . . . . . . . . . . 32

3.4 Coupled Theories . . . . . . . . . . . . . . . . . . . . . . 33

3.4.1 Simple Scalar Damage (oupled with Plasticity . . . . . . . . 34

3.4 .2 vol Mises . . . . . . . . . . . . . . . . . . . . 35

3.4 .3 Principal Stress . . . . . . . . . . . . . . . . . . 35

4. BIFURCATIONS AND LOCALIZATION . . . . . . . . . . 37

4.1 Introduction to Bifurcation (riteria . . . . . . . . . . . . . . 37

4.1 .1 General Bifurcations . . . . . . . . . . . . . . . . 39

4.1.2 Limit Point Bifurcations . . . . . . . . . . . . . . . 40

1.1.3 Classical Discontinuous Bifurcations; . . . . . . . . . . . 40

4.1.4 Loss of Strong Ellipticity . . . . . . . . . . . . . . . 41 
4.1.5 Summary of Bifurcation ('riteria . . . . . . . . . . . . 42

1.2 Characterization of Bifurcation Modes . . . . . . . . . . . . . 13

4.3 Bifurcation Criteria and the Eigensystem for $\mathbf{D}^{s} \quad$. . . . . . . . . . . 45

4.4 Eigenanalysis of the Elastic Tangent Stiffness Tensor . . . . . . . . . 46

4.5 Eigenanalysis of the Plastic Tangent Stiffness Tensor . . . . . . . . . . 17

4.6 Bifurcation Analysis of Plasticity Models . . . . . . . . . . . . . . . 49

4.6 .1 Drucker-Prager . . . . . . . . . . . . . . . . . . . . . . . . 4

4.6 .2 von Mises . . . . . . . . . . . . . . . . . . . . . 5.3

4.6 .3 Mohr-Coulomb . . . . . . . . . . . . . . . . . . . . 5.5

4.6 .4 Principal Stress . . . . . . . . . . . . . . . . . . . . 56

4.7 Evaluation of von Mises Plasticity for Metals . . . . . . . . . . . . . 57

4.7 .1 Axisymmetric Rod Subjected to Intaxial Tension . . . . . . 57

4.7.2 Axisymmetric Rod with Lateral Displacements Constrained . . . 58

4.7.3 Thin Plate Subjected to Iniaxial Tension . . . . . . . . . . 59)

4.7.t Thin Plate Subjected to Equal Biaxial Tension . . . . . . . 61

4.7.5 Thin-Walled Cylindrical Pressure Vessel . . . . . . . . . . $\quad$ (i.3

4.8 Bifurcation Analysis of (Continum Damage Models . . . . . . . . 61

4.8 .1 Simple Scalar Damage . . . . . . . . . . . . . . . . . . 6.5

1.8.2 von Mises Damage . . . . . . . . . . . . . . . . . 65

1.8.3 von Mises Damage with Alternate Damage Evolution . . . . . $\quad 66$

4.8 .4 Principal Stress Damage . . . . . . . . . . . . . . . 67

4.8.5 Principal Strain Damage . . . . . . . . . . . . . . . . 6 .

5. NUMERICAL SIMULATION OF LOCALIZATION . . . . . . . (i)

5.1 Analyses with Existing Finite Element Codes . . . . . . . . . . 69

5.1.1 Rod Subjected to Iniaxial '́ension . von Mises Plasticity . . . . 69)

5.1.2 Rod Subjected to Iniaxial 'Tonsion - Principal Stress Plasticity . . T2

5.1 .3 'Thin Plate Subjected to Iniaxial T'onsion - von Mises Plasticity' . T2

5.1 .4 Thin Plate Subjected to Equal Biaxial 'Tension . . . . . . . T5

5.2 Modifications to Obtain Numerical Solutions . . . . . . . . . . . . T

5.3 Modifications to Eliminate Mesh Dependence . . . . . . . . . . Ts

5.4 Elements with Embededed Localized Deformation zone . . . . . . . . . T9 
6. A MODEL FOR POLYURETHANE FOAM . . . . . . . . . . . 85

6.1 Description of the Foam as a Mixture . . . . . . . . . . . . . . . . 85

6.2 Air/Polymer Response . . . . . . . . . . . . . . . . . . . . . . . 86

6.3 Plasticity Theory for the Skeleton . . . . . . . . . . . . . . . . . 89

6.4 Coupled Theory for the Skeleton . . . . . . . . . . . . . . . . . . . 89

6.5 Simplified Isotropic Theory for the Skeleton . . . . . . . . . . . . . . . . 90

6.6 Analysis of Material Characterization Tests . . . . . . . . . . . . . . 91

7. ALUMINUM HONEYCOMB MODEL . . . . . . . . . . . 97

7.1 In-plane Response . . . . . . . . . . . . . . . . . . . . 97

7.2 Generator Axis Response . . . . . . . . . . . . . . . . . 101

7.3 A Baseline Model . . . . . . . . . . . . . . . . . . . . 101

8. FINITE ELEMENT ANALYSIS OF AN IMPACT EVENT . . . 103

9. CONCLUSIONS AND FUTURE WORK . . . . . . . . . . . 109

10. REFERENCES . . . . . . . . . . . . . . . . . . . . 111 


\section{Figures}

1.1 (Cllular Solids: (a) alumimm honeycomb, (b) polyurethane foam. . . . II

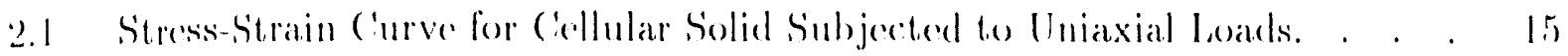

2.2 Failure Surlare for Regular Hexagonal Honeycomb Subjected to In-plane Lads (Gibson and Ashby, l988). . . . . . . . . . . . . . . 17

2.3 Localized In-planc Deformation of Alumimum Iloneycomb Subjected to llniaxial (ompression: (a) in the 'Transverse Direction, (b) in the Ribbon Di-

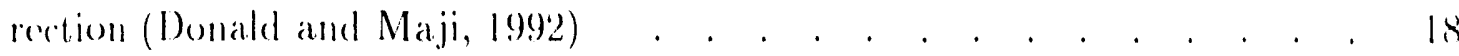

2.1 Failure Surfare for a Rigid, ('losedecedl, Polyurethand Foam (Paicel and Finnic, l969); (iibson and Ashby, 1988). . . . . . . . . . . . . 1!

2.5 loal-1)isplacement ('urve for $20 \mathrm{lb} / \mathrm{ft}^{3}$ Polyurethane Foam Subjected to ('yclic Iniaxial ('ompression (Donald and Maji, 1992). . . . . . . . 20

3.1 Drucker-Prager Yield Surface. . . . . . . . . . . . . . . . 2 26

3.2 von Mises Yicld Surface. . . . . . . . . . . . . . . . . . 27

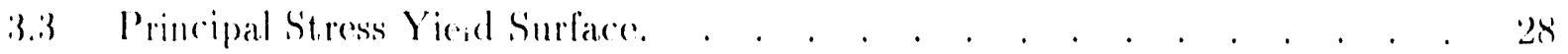

4.1 Discontinnons Bifuration Modes. . . . . . . . . . . . . . . 41

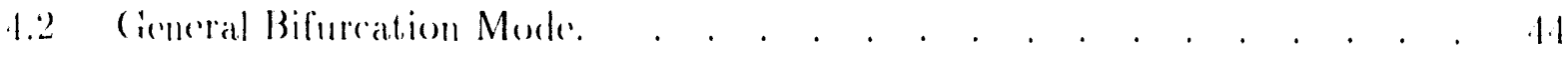

1.3 Criticalltardening Moduli for a Druseker-Prager Material with Non-Associated flow. . . . . . . . . . . . . . . . . . . . . 51

1.1 ('ritical Hardening Moduli for a Drucker-Prager Material with Assoriated Flow. . . . . . . . . . . . . . . . . . 5.3

4.5) Bifurcation Regimes for a von Mises Material Subject to Hniaxial lemsion. 5r.

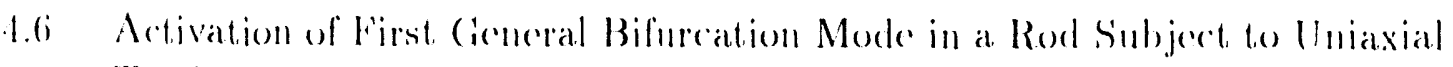
'lomsion. . . . . . . . . . . . . . . . . . . . . 5 . 5

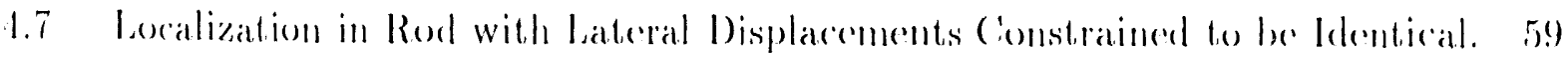

t.x Artivation of (ieneral Bifureation Mode in a Plate Subject to Uniaxial 'Pension. (io)

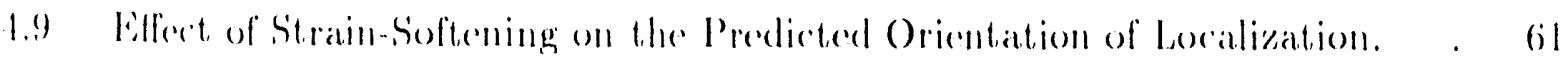

1.10 Artivation of Discontinums Bifurcation Mode in a Thin Pate Subject tes

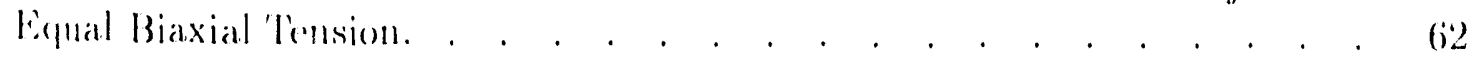

1.11 Artivation of a Discontinuous Bifuration at the Limit Point in a Pressitrized Cylindrical Pressure Vessol. . . . . . . . . . . . . . . . . 6 (i.3

5.1 Axisymmetrie Finde Element Models of a Rod. . . . . . . . . . 70

5.2 Deformed Shape of Finte Element Models Subject to an Axial Displacement of 0.10 in. - Elastic, Perfectly Plastic von Mises Material. . . . . 71

5.3 Applied Load vs. End Displacement - Elastic, Perfectly Plastic von Mises Malcrial. . . . . . . . . . . . . . . . . . . . . . 71

5.4 Results from Rinite Element Analysis with $\mathrm{H}=-\mathrm{E} / \mathrm{L2} . \quad$. . . . . . . . 73

5.5) Results from Finite Blement Analysis with $\mathrm{H}=-\mathrm{E} /(6-6 \mathrm{v})$. . . . . . . . 73 
5.6 Deformed Shape of Finite Flement Models Subject to Iniaxial Tension Principal Steress Plasticity. . . . . . . . . . . . . . . . . . . . . . 7.1

5.7 Applied load vs. End Displacoment - Primcipal Stress Plasticity. . . . TA

5.8 Finite Element Models of a Thin Plate. . . . . . . . . . . . . T5

5.9 Normal Displacement ('ontoms for a Thin Plate Subjected to Hniaxial Tension - 576 Element Mesh. . . . . . . . . . . . . . . . . . 76

5.10 Normal I)isplacement ('ontours for a Thin Plate Subjected to Oniaxial Tension - 3456 Element Mesh. . . . . . . . . . . . . . . . . 76 ;

5.11 Normal Displacement (ontours for a Thin Plate S'ubjected to Equal Biaxial Tension. . . . . . . . . . . . . . . . . . . . . . . . 7 个

5.12 Loal-1)isplacement ('urve Exhibiting Snap-Back. . . . . . . . . . TS

5.13 Soltening Zone in Elastic--Plastic Rod Subjected to Tniaxial Tension. . . So)

5.14 ('onstitutive Relation for Elastic-Plastic Material. . . . . . . . . . .

5.15 Applied Load vs. End Displacement Predictions Obtained Using Finde Elements with Embededed Softening Zones. . . . . . . . . . . . . S1

5.16 Constitutive Rolation for Elastic-Damaging Material. . . . . . . . . 82

6.1 Decomposition of Foam Response into Skeleton and Air/Polymor Responses. sot

6.2 Air/Polymer Response for a 20 pef Polyurethane Foam. . . . . . . . sis

6.3 Air/Polymer Response for a 20 pef Polyurethane Foam with Air Allower to Escrape. . . . . . . . . . . . . . . . . . . . . . . 88

6.4 Axisymmetric Finite Element Models of Foam Specimens. . . . . . . 9:3

(j.5) Deformed Shape of 200 Element Model Subject to Iniaxial ('ompression. . 93

6.6 Applied Load vs. End Displacement - Uniaxial ('ompression - Comparison of Finte Element Analyses Predictions with Experimental Observations. . 94

6.7 Deformed Shape of Models Subject to Iniaxial Tension. . . . . . . 94

6.s Applied Load vis. End Displacement - Lniaxial Tension - ('omparison of Finite Element Analyses Predictions with Experimental Observations. . . 95

(b.9) Doformed Sibape of Models Sibbject to Hydrostatic ('ompression. . . . . 9.5

6.10 Applied Pressure vs. Enginerering Volume Strain - Hydrostatic Compression - (omparison of Finite Element. Analyses Predictions with Experimental Observations. . . . . . . . . . . . . . . . . . . . . . 96

T.l In-plane Loading of Aluminum Honeycomb cells. . . . . . . . . . . 98

7.2 ('omparison of Bifurcation Analysis Predictions with Experimental Observations of Localization in Aluminum Honeycomb Subjected to Jniaxial ('ompression in the $x$-Direction. . . . . . . . . . . . . . . .

7.3 (omparison of Bifurcation Analysis Predictions with Experimental ()bservations of Localization in Aluminum Honeycomb Subjected to Iniaxial Compression in the $y$-Direction. 
7.4 Localized Deformation Exhibit by Alumimum Honeycomb Subjected to Hni. axial (ompression Along its Generator $A$ xis. . . . . . . . . . . 101

7.5 Yield Surface for Aluminum Honeycomb. . . . . . . . . . . . I02

8.1 Finite Element Model Used in Dynamic Analyses. . . . . . . . . 10:3

8.2 Deformed Shape of Finite Element Model at Maximm (Trush-Hp. . . . Iot

s.3 Displacement of Siteel Cylinder Predicted by the Analyses. . . . . . . 107

8.4 Velocity of Steel cylinder Predieted by the Analyses. . . . . . . . . 107

8.5) Acoeteration of Steed (Eylinder Predieted by the Analyses. . . . . . . 107

s.6 Damage in Foam Layer Predicted with ('oupled (onstitutive 'Theory. . . Ios

\section{Tables}

4.1 Simmary of Bifurcation (iriteria. . . . . . . . . . . . . . . . . 42

5.1 von Mises Material Parameters. . . . . . . . . . . . . . . . 6i)

5.2 Principal Steress Material Parameters. . . . . . . . . . . . . . T2

(i.) Material Parameters for a 5 pef Polyurethane Foam. . . . . . . . . 91

7.1 Parameters for a 0.125-5052-002 Alumimum Honcycomb. . . . . . . .99

s.l Material Parameters lsed in Finite Element Analyses. . . . . . . . 105 


\section{INTRODUCTION}

Cellular materials consist of interconnected plates or struts which form cells. The struts or plates are constructed from a variety of metals, polymers, ceramics and wood products. Materials with a two-dimensional array of cells are referred to as honeycombs and materials with a three-dimensional array of cells are referred to as foams (Figure 1.1). Foams with interconnected plates often form closed-cells and are referred to as closed-cell foams. Foams with interconnected struts are referred to as open-cell foams and are generally much more flexible than closed-cell foams. Foams that permanently deform when subjected to large strains are rigid foams and foams that remain elastic for large strains are flexible foams. The two materials that were closely investigated in this study include a rigid, aluminum honeycomb and a rigid, closed-cell, polyurethane foam.
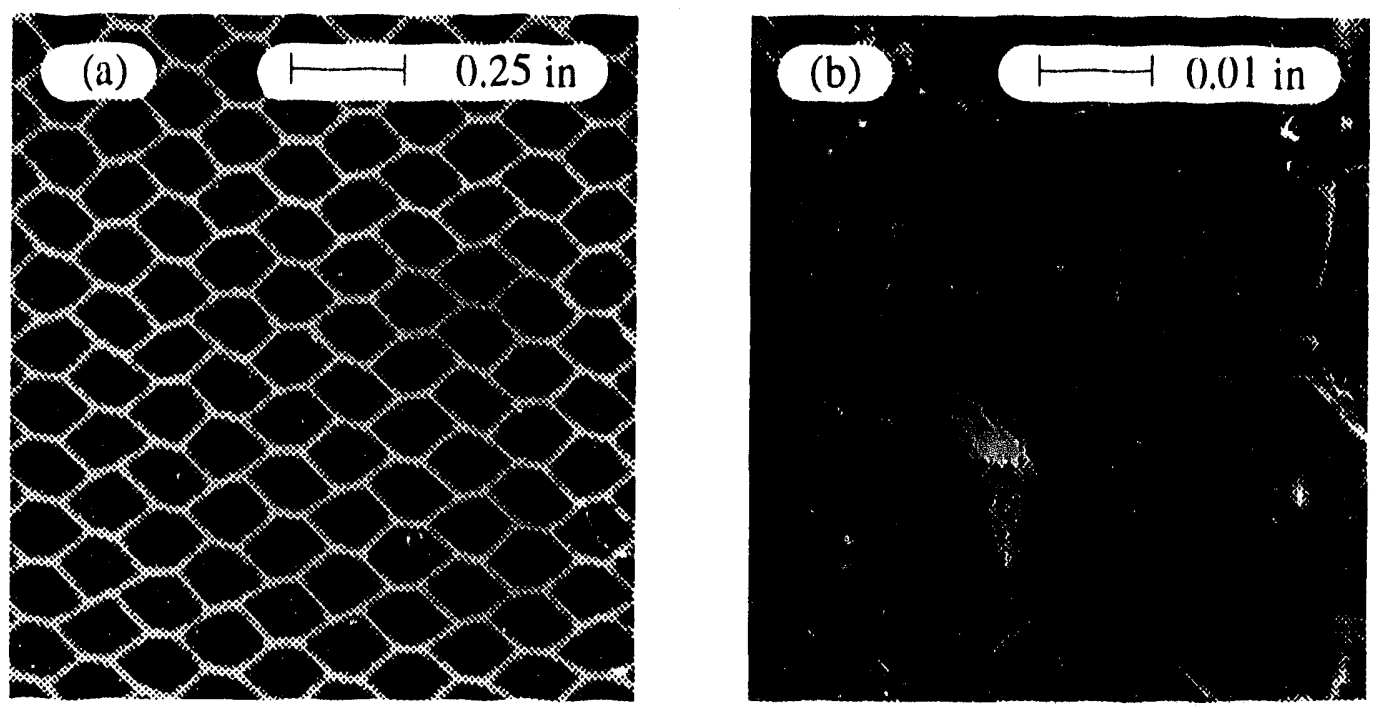

Figure 1.1. ('ellular Solids: (a) aluminum honeycomb, (b) polyurethaue foam.

('ellular materials have been studied by mathematicians and philosophers for over 300 years (Cibson and Ashby, 1988). Early mathematicians were intrigued by the geometry and packing of individual cells. (ellular solids have a variety of cell shapes and packing arrangements. For example, aluminum honeycomb has regular hexagonal cells and a uniform packing. On the other hand, polyurethane foam has a number of different cell shapes and packing arrangements. For the past few decades, researchers have experimentally measured and analytically investigated the mechanical behavior of cellular solids. The experimental work has included subjecting cellular solids to various load paths and measuring the induced deformation of the sample. The analytical work has concentrated on developing equations that relate the elastic response and initial yield 
strength of the cellular material to the cell geometry and the properties of the cell wall material. These investigations indicate that cellular solids have mechanical properties which are not typical of solid materials.

('ellular solids are used in a wide vartedy of applications that take advantage of the muigure pepertices of these materials. For example, collular solids are used as fillers and rigirlizers

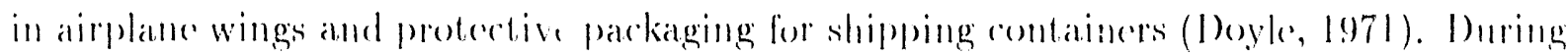

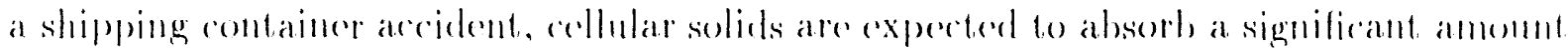

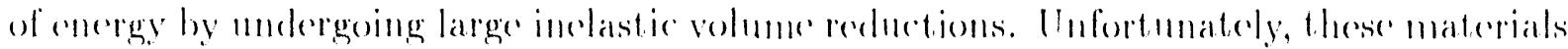

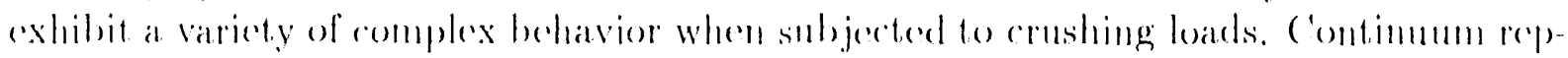

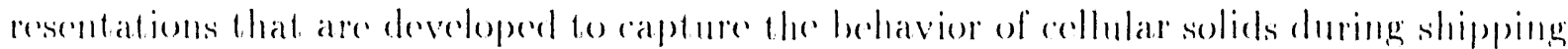
container arcidents must capture these complex hehavior.

The purpose of this rescarch was to develop a contimum representation of cellular matlerials that not only captures the elastic response of these materials but also the phastie deformation, damage and localized deformation that is generated when these materials are subjected to crushing loads. The constitutive relations developerd for these materials nse a combination of plasticity and comtimumm damage theories. Plasticity is a well devel. opeed theory which was originally used to describe the permanent deformations of metals loaked heyond their elastice limit. Rerently, plasticity has also beren used to drscribe the permanent deformations exhibited by concerte, rocks, soils and foams. ('ontimmm damage theories, on the other hand, are just corrently heing developerd (liajocinovic, 1959). In this papere, a rather general comtimmm damage theory with a stertucture similar to existing plasticity theories is presented. Ise of a similar structure simplifies the develepment of conpled plasticity-contimum damage theories which shate common yiold (damage) surfares. In this study, a compled theory is developed for rigid polyurethane foam.

When rellular solids are loaded into the inelastic regime, they often exhibit deformations that are localized into small regions at some point in the loading process. In the past, localization has been associated with loss of positive definiteness of the aconstic tensor (Hill, 1962; Rice, 1976). Here we show that localization should be associated with loss of positive definiteness of the symmetric part of the acoustic tensor. The acoustic tensor depends on both an orientation vector for the localization and on the fourth-order tangent stiffness tensor for the material. A connection between constitutive theories and localization is provided by a spectral analysis of the symmetric part of the tangent stiffness tensor. The eigensystem for the symmetric part of the tangent stiffness tensor is obtained for several different plasticity and continuum damage models. This eigensystem provides information about both diffuse and discontinuous bifurcations. Material properties, boundary conditions, and body geometry are all shown to affect the diffuse and localized deformation modes that are generated. Numerous experimental observations of necking and localization in metal specimens subject to various boundary conditions are explained with a bifurcation analysis of a von Mises plasticity model. Bifurcation analyses are then performed on potential constitutive theories for cellular solids. A comparison 
of results from the bifureation analyses with experimental observations of localized deformations is essential for the identification of appropriate constitutive theorices for collular materials.

('onstitutive relations for aluminmm honeycombs and rigid polyurethane foams are developed and analyzed. A coupled plasticity and continuum damage model for rigid polyurethane foam is then implemented in both static and dynamic finite element codes. A number of static tests on rigid polyurethane foam are analyzed and the results from the finite element analyses are compared with experimental observations. Finally, a typical impact problem is analyzed using various constitutive models for a polyurethane foam impact limiter to demonstrate the effects of using various constitutive descriptions for the foam. 


\section{CELLULAR MATERIALS}

Literature on the mechanical behavior of cellular material is reviewed in this chapter. The first section includes a discussion on the general behavior of cellular material. In the second section, currently available experimental data for aluminum honeycomb and rigid, polyurethane foam is reviewed. In the final section, existing constitutive theories for these materials are reviewed and the need for improved constitutive theories is discussed.

\subsection{General Behavior}

(ellular materials generally have a much lower density, thermal conductivity, stiffness, and strength than solid materials (Gibson and Ashby, 1988). A typical stress-strain curve generated from a uniaxial compression test on a cellular solid is shown in Figure 2.1. Initially the cellular solid is linear elastic. When load is applied the cell walls bend but remain linear elastic. As the load is increased, the cell walls begin to elastically buckle, form plastic hinges, or crack. The behavior depends on the cell wall material, temperature, and loading rate. Generally, one layer of cells will completely collapse before a second layer collapses. This generates a narrow region of localized deformation. In this plateau region, a small increase in the applied load will cause additional layers of cells to collapse and the region of localized deformation will continue to grow. It is exactly this behavior that makes cellular materials attractive for use in impact limiters. Once a

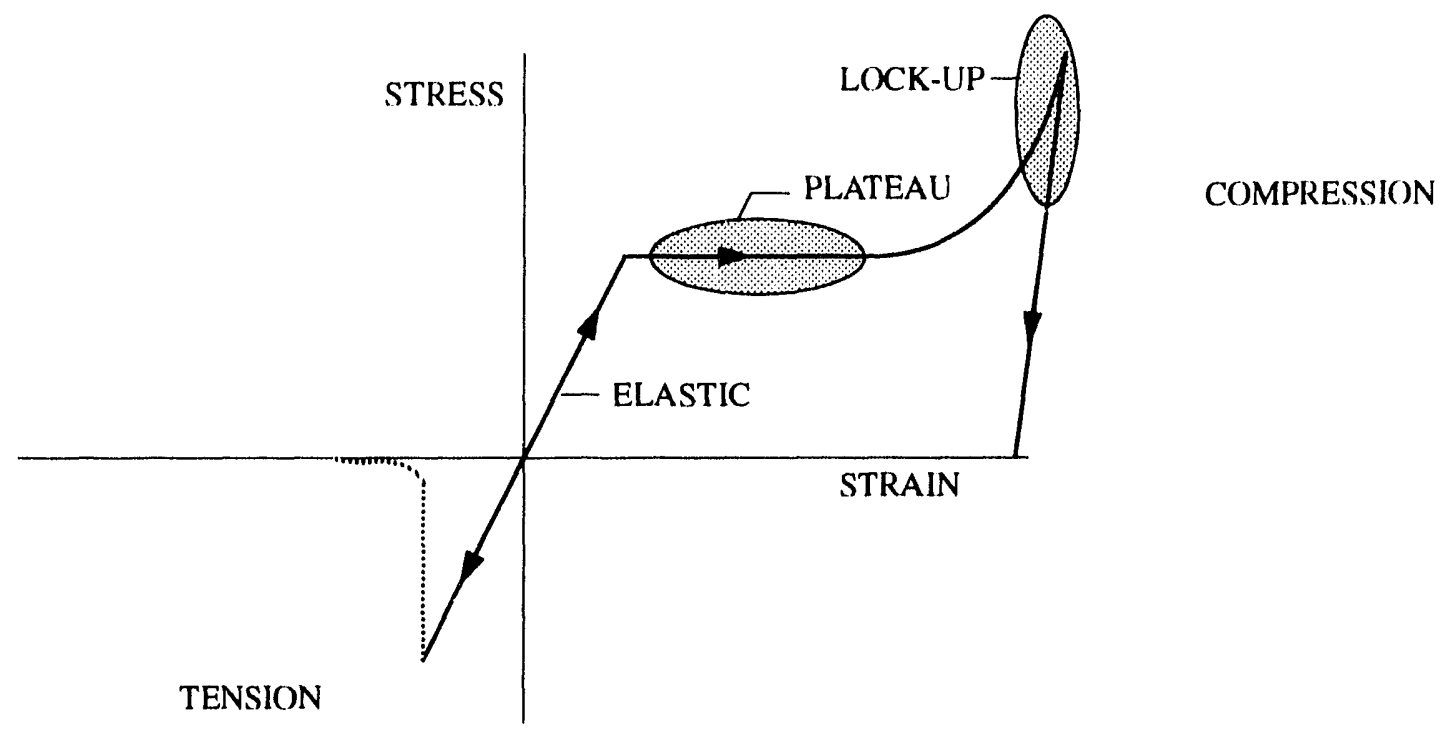

Figure 2.1. Stress-Strain Curve for Cellular Solid Subjected to Uniaxial Loads. 
certain load level is reached, the material experiences large volume reductions and absorbs a significant amount of energy with only a small increase in transmitted load. Finally, once all of the cell rows have collapsed, the cell walls are compressed against each other and the load needed to compress the sample further will rapidly increase. This is referred to as densification or lock-up of the cellular solid. The unloading behavior will depend on the character of the cellular material. Flexible cellular materials will elastically unload and return to their undeformed shape. Rigid cellular materials, on the other hand, are permanently deformed and often damaged when they are compressed into the plateau regime. This study focuses on rigid cellular solids which exhibit inelastic deformations.

When cellular solids are subjected to tensile loads, the cell walls will again bend and the material will initially be elastic. As the load is increased, the material will either plastically deform or fracture. The behavior will again depend on the cell wall material, temperature and load-rate. If the material fractures, the applied load will rapidly decrease. If the material plastically deforms, the load will increase as the cell wall sections are rotated and axially stretched. Often a single row of cells will stretch or fracture and the material will also exhibit localization in tension.

\subsection{Experimental Data}

Two cellular materials that are often used in packaging, aluminum honeycomb and rigid polyurethane foam, have been experimentally evaluated by a number of researchers (Shaw and Sata, 1966; Patel and Finnie, 1969; Zaslawsky, 1973; Klintworth and Stronge, 1988; (iibson and Ashby, 1988; Donald and Maji, 1992). Results from these experimental investigations are briefly summarized in this section.

\subsubsection{Aluminum Honeycomb}

Aluminum honeycomb consists of interconnected aluminum plates. The plates are connected such that they form hexagonal cells that resemble a bee's honeycomb. This material is not isotropic. In fact, the strength of the material in a direction parallel to the generator axis is approximately 30 times greater than its in-plane strength.

A typical yield surface for an aluminum honeycomb subjected to in-plane loards (Gibson and Ashby, 1988) is shown in Figure 2.2. When this material is subjected to a uniaxial in-plane compressive load the cell walls bend, plastic hinges form near the connection between various cell walls, and cell walls eventually deform and collapse. Generally, a localized region of cells will collapse and begin to densify before cells in other regions collapse. Figure 2.3 shows the localized deformations generated by uniaxial compression in the ribbon direction and uniaxial compression in the transverse direction. The localized deformation zones in these materials are generally oriented along geometrically similar lines. However, the localized deformation is significantly different when the load orientation is changed. Thus, the constitutive description for aluminum honeycomb should 


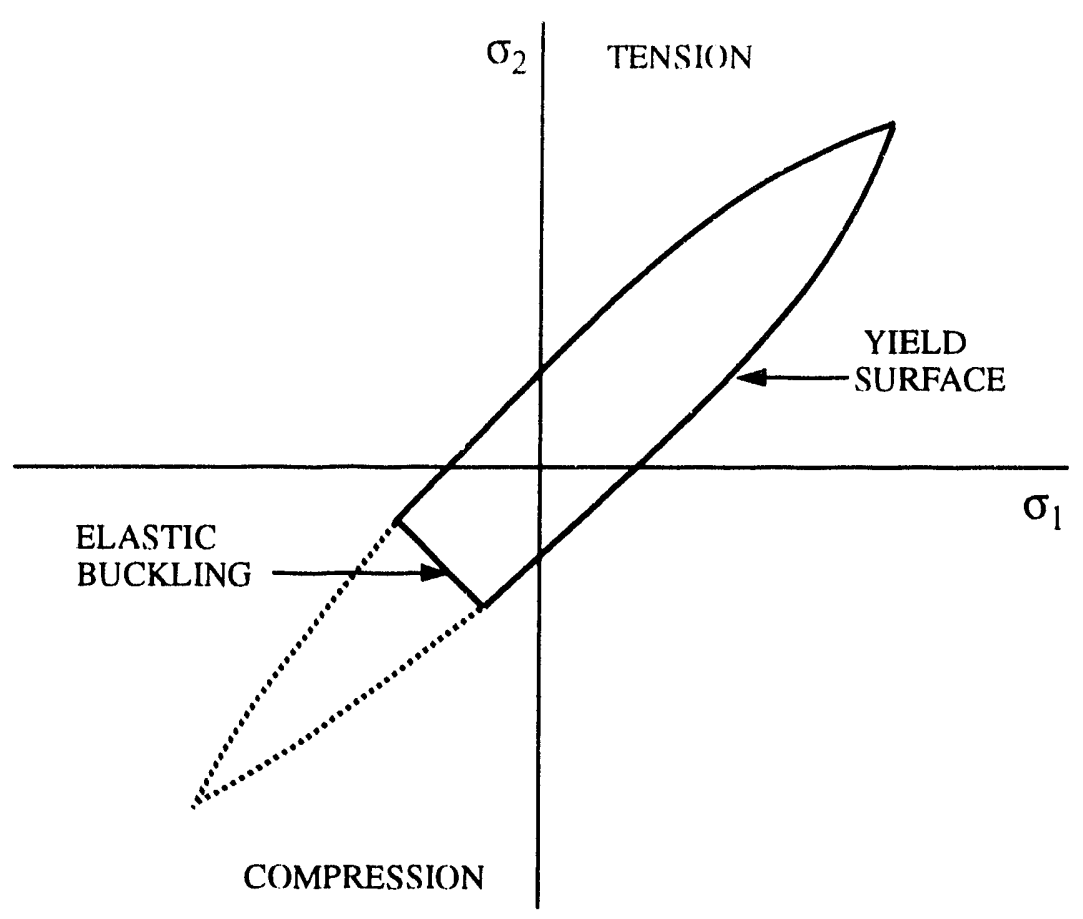

FiŁure 2.2. Failure Surface for Regular Hexagonal Honeycomb Subjected to In-plane: Loads (Gibson and Ashby, 1988).

predict geometry and load dependent localized deformation.

For uniaxial compression, the load-displacement curve consists of an initial linear elastic regime followed by a platean regime where the cells collapse as plastic hinges form in the cell walls. Finally, a regime of densification is reached where all of the cells have collapsed and begin to densify which causes the load carried by the structure to rapidly increase. The behavior of this material under uniaxial tension is similar to its behavior under uniaxial compression. Plastic hinges form near the connection between various cell walls and cell walls bend and stretch. Again, a localized region of cells will stretch and ine elongated before cells in other regions are plastically deformed. Eventually the entire matrix is elongated and the response is equal to the response of the solid cell wall material. The cross section of the sample has, of course, been significantly redured.

When this material is subjected to uniform biaxial tension, the cell walls are stretched and not bent. Note that the yield surface (Figure 2.2) for aluminum honeycomb is elongated along the biaxial load path. This occurs because the biaxial load needed to stretch the cells walls is significantly higher than the uniaxial load needed to bend the cell walls. Since the permanent deformation exhibited by this material is associated with the formation of plastic hinges in the cell walls, a plasticity model is used to capture the inelastic behavior. 

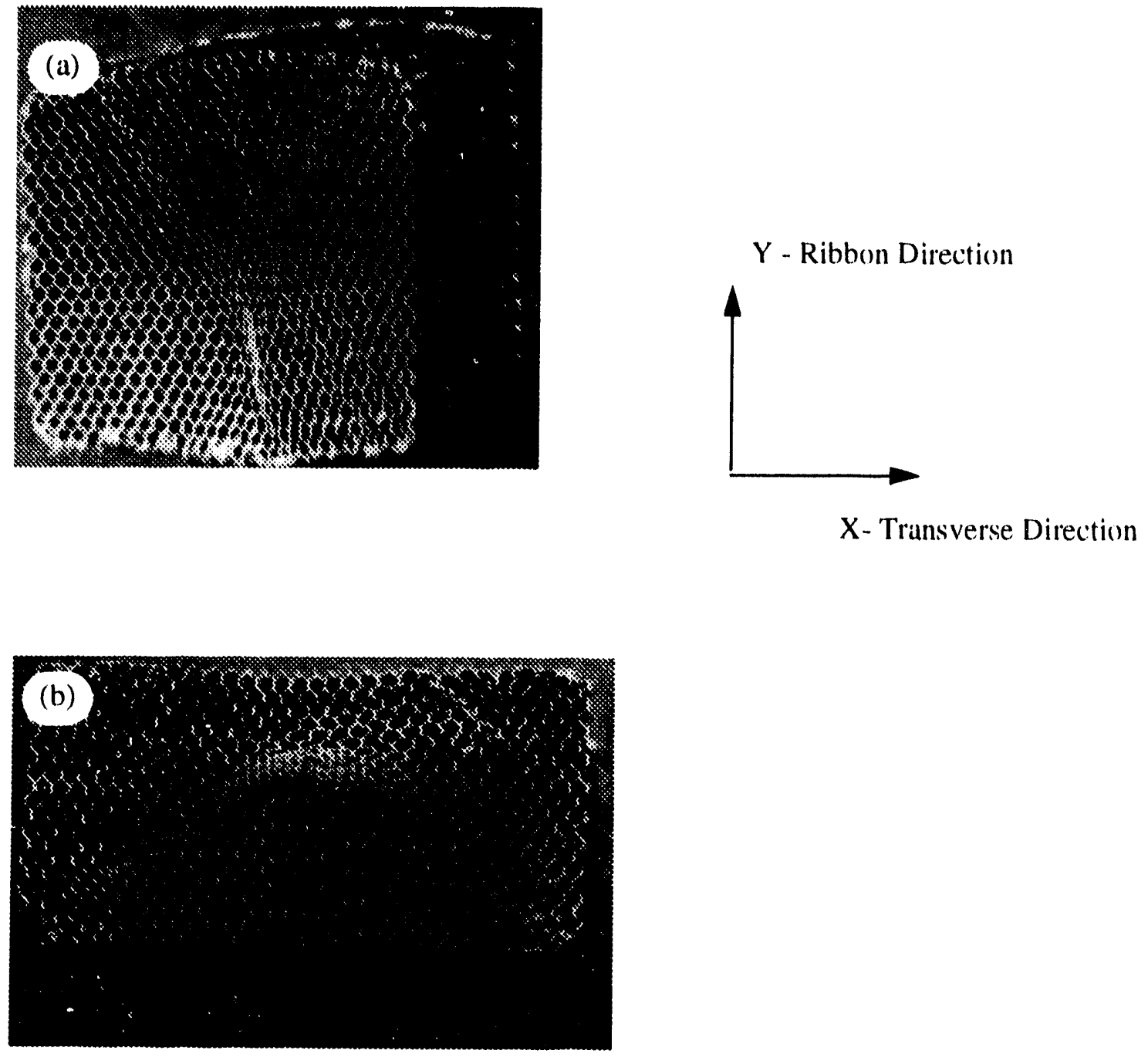

Figure 2.3. Localized In-plane Deformation of Aluminum Honeycomb Subjected to Iniaxial (ompression: (a) in the Transverse Direction, (b) in the Ribbon Direction (Donald and Maji, 1992) 


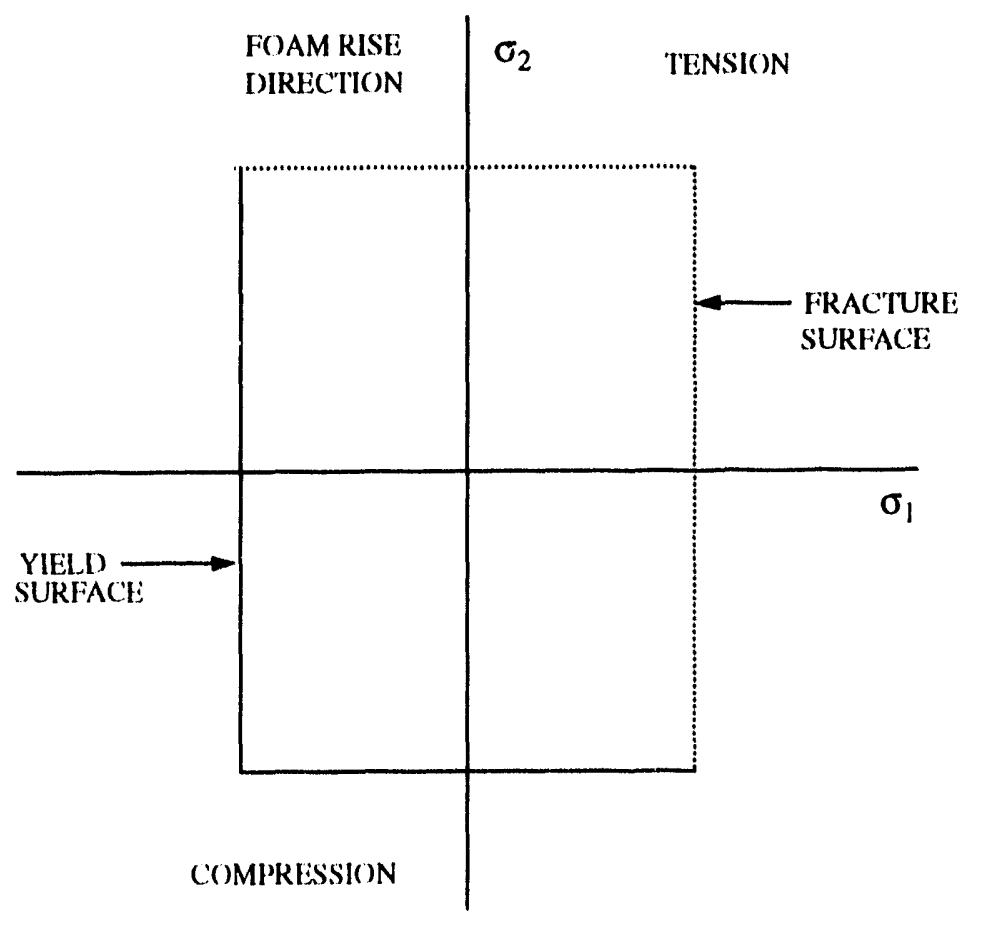

Figure 2.4. Failure Surface for a Rigid, ('losed-Cell, Polyurethane Foam (Patel and Finnie, 1969; (ibson and Ashby, 1988).

\subsubsection{Polyurethane Foam}

Rigid, closed-cell polyurethane foam consists of interconnected plates of polyurethance. The geometry of this material is three-dimensional and the plates are much more randomly distributed than the plates in aluminum honcycomb. This material is manufartured hy having a gas expand inside liquid polymer which solidifies in a foamed state. Shaw and Sata (1966), Patel and Finnie (1970), Zaslawsky (1973), and Donald and Maji (1992) have experimentally measured the behavior of polyurethane foam samples subjected to varions load paths. Patel and Finnie's (1969) work indicates that the initial failure surface for this material subjected to a plane stress state has the rectangular shape shown in Figure 2.4. The experimental work of Shaw and Sata (1966), Zaslawsky (1973), and Donald and Maji (1992) indicates that the initial yield surface for rigid polyurethane foam can be described using a principal stress criterion.

A load-displacement curve for $20 \mathrm{lb} / \mathrm{ft}^{3}$ polyurethane foam subjected to cyclic uniaxial compression is shown in Figure 2.5. The initial elastic regime is followed by a platean regime in which the load required to crush the material remains nearly constant. In this regime, some cell walls are compressed until they buckle while other cell walls are stretched until they crack. When additional load is applied, the cell walls are compressed against other cell walls and the stiffuess of the foam approaches a value coual to the stiffness of solid polymer. The slopes of the unloading curves indicate that the material stiffness decreases when the material is compressed into the platean regime but that the 


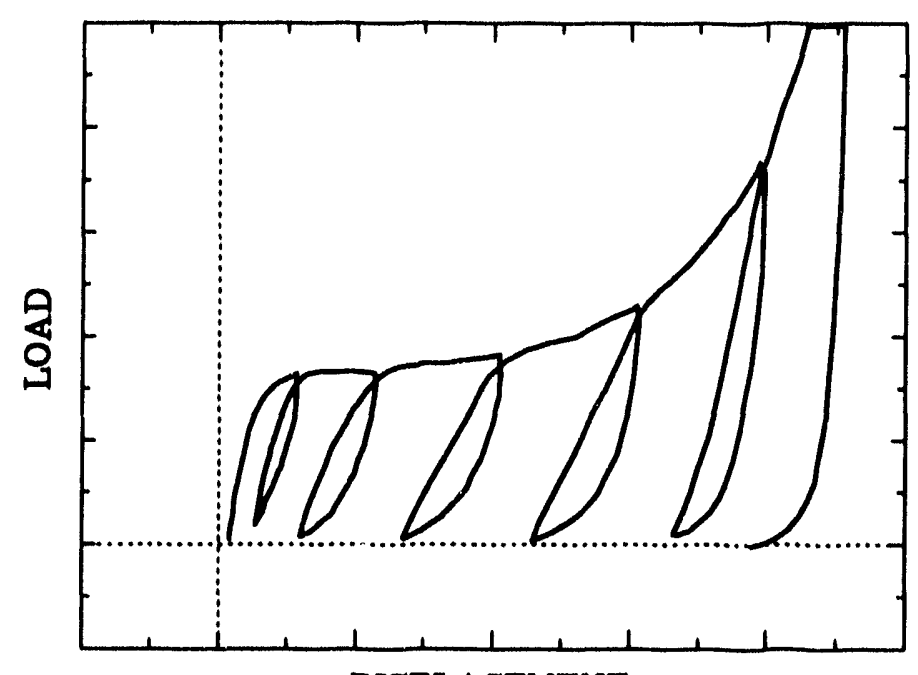

DISPLACEMENT

Figure 2.5. Load-Displacement ('urve for $20 \mathrm{lb} / \mathrm{ft}^{3}$ Polyurethane Foam Subjected to ('yclic Uniaxial (ompression (Donald and Maji, 1992).

material stiffuess actually increases when the material is compressed further into the densification regime. The decrease and subsequent increase in foam stiffness is cansed by two distinct deformation mechanisms. The decrease in stiffness occurs due to the cracking and reduced stiffness of the cell walls. The increase in stiffuess is due to the interaction between neighboring cell walls as they are compressed together.

\subsection{Existing Continuum Representations}

Most packages are currently designed using a one-dimensional uniaxial crush model for the cellular solid. This is done due to the lack of arlecuate experimental data or an accurate constitutive theory for these materials. In a typical design, the miaxial loaddisplacement curve is used directly to compute the amount of energy absorled by the foam for a given uniaxial impact event. This method is accurate for describing the response of impart limiting materials subject to miaxial loads; however, this simple uniaxial approach cannot be applied to situations involving multiaxial loading. A general continum representation is needed if multiaxial loading is involved. The uniaxial crush model is adequate for only the simplest of parkage geometries and loadings.

A variety of researchers (Cibson and Ashby, 1988; Warren and Kraynik, 1987) have studied the linear elastic behavior of various cellular solids. In this work, equations are developed that relate the elastic constants to parameters that define the geometry of the cell (i.e., the thickness of the cell wall and length of a typical cell wall section). Equations that define the critical load beyond which the behavior is no longer linear elastic are also developed by these rescarchers. These equations are then used to describe the 


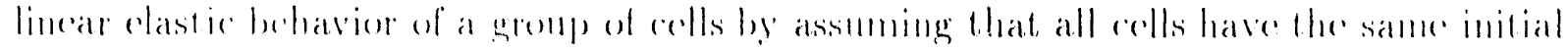

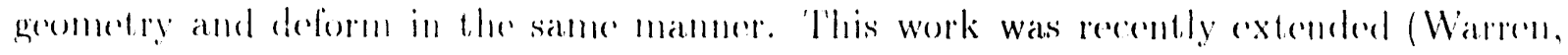
Laynik and Stone, 1989) to apture the nonlinear elastic behavior of two-dimensional open cell foams. Results from these micromechanical investigations indicate that the dominant cell deformation merhanism in flexible foams changes from hending for small deformations to stretehing for large deformations.

The micromechanical studies of Warren and kraynik (1987) and others provide adeseription of the cellular material behavior that is based on an understanding of the helavior at. the cell level. These studies provide valuable information about rell deformation menanisms and the effects of cell geometry or material variations on the global response of the foam. This is important because it allows for the development of an engineered collular material that meets specific: global behavior requirements. Current micromechanical studies arcurately capture the linear and nonlinear elastic hehavior of cellular materials that have a regular cell geometry. However, a significant extension of the current. micromechanical studies would be needed to investigate the effects of irregular cells, to predict inclastic behavior, or to capture localized deformation. Extension of micromechanical studies to ine astic behavior and localization will probably require a complement of analytical and mumerical investigations of families of cells.

In this research, a maremechanical approach is used to investigate the behavior of cellular solids. With this approach an extensive experimental investigation is necoled to evaluate the deformation and localization exhibited by a large number of cells subjected to varions load paths. Results from the experimental investigations are then used to dovelop a mathematical description of the behavior. This approach allows the development of a model that captures both the elastic and inclastic behavior of the material. $\Lambda_{11}$ arecurate description of ach individual cell is not needed; thus, this approach works well for materials that have irregular cells like polyurethane foam. Infortumately, if the equations are independent of eell geometry then the efferet of variations at the coll level cantent he related to variations in global hehavior. With this approach localized deformations must be measured during experiments if a model that aptures localized deformation is to loe developed. Also a thorongh understanding of the relationship between localized deformations and constitutive descriptions is needed to develop appropriate constitutive theories for materials exhibiting localization. A significant portion of this work is a development. of such an understanding.

Most rommereial finite element codes do not have comstitulive theories for cellular solids and analysts using these codes are foreed to use an inappropriate comstitutive description for collular materials when they are laced wilh a problem in which these materials are used. A few rescarchers are corrently working on the development of constitutive the ories for collular materials (Noilsen, Morgan and Krieg, 1987; Klintworth and Stronge, 1988; Warren, Rraynik and Stone, 1989; Triantafillon and (iibson, 1990) and this report. represents some of that work. Recently, Klintworth and Stronge (1988) investigated the response of aluminmm homeycombs subjected to in-plance loads and developerd mathemat- 
ical expressions for the elastic response and the initial yield functions for this material. Their yield functions describe a family of intersecting yield surfaces in stress spare. 'The yield functions for aluminum honeycomb developed by Klintworth and Stronge (1988) are used in the plasticity theory for aluminum honeycomb presented here. A plasticity model for rigid polyurethane foam, developed by Neilsen, Morgan and Krieg (1987), is used as the basis for the coupled plasticity continumu damage model for polyurethane foam developed in this study. 


\section{CONTINUUM CONSTITUTIVE THEORIES}

This chapter includes a review of elasticity, plasticity and continumm damage theories which may be useful for describing the behavior of cellular solids. The continumm damage theory was developed by Sichreyer (1990a) using a structure similar to that of conventional plasticity theory. The use of this structure ensured the development of thermodynamically areceptable constitutive theories and also aided in the identification of continumm damage theories which are analogous to existing plasticity theories. This was helpful in the development of coupled (plasticity with continum damage) theories which have common yiedd (damage) surfaces.

\subsection{Elasticity}

Elasticity theories were developed to deseribed the deformation of materials not loaded beyond their elastic limit. For a class of linear clastic materials, there exists a positive definite strain energeg per unit volume, 1!, (Malvern, 1969), given by

$$
l=\frac{1}{2} \epsilon: \mathbf{E}: \epsilon
$$

where $\epsilon$ is the total strain tensor and $\mathrm{E}$ is the fourth-order elastic stiffuess terlosol. All of the fourtherder tensors used in this report possess the minor symmetries, i.e., $E_{i j k l}=$ $E_{, 2 k l}=E_{i, l k}$. The existence of a strain energy function implies that the elastic stilluess tortsol also possessess the major symmotry, i.e., $E_{i j k l}=E_{k l i j}$.

For small deformations, the constitutive relation is as follows:

$$
\boldsymbol{\sigma}=\frac{\partial \eta !}{\partial \epsilon}=\mathbf{E}: \boldsymbol{\epsilon}
$$

If the material is isotropic, then $\mathbf{E}$ is given by

$$
\mathbf{E}=3 K \mathbf{P}^{s p}+2 C \mathbf{P}^{d}
$$

where $K$ is the bulk morlulus and $(i$ is the shear modulus. The bulk and shear moduli

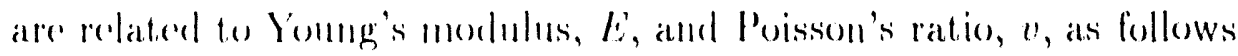

$$
K=\frac{E}{3(1-2 v)} \quad\left(i=\frac{b}{2(1+v)}\right.
$$

The fourth-order spherical projection operator, $\mathbf{P}^{\text {sp }}$, and the deviatoric projection operator. $\mathbf{P}^{\prime}$, are given by

$$
\mathbf{P}^{s \prime}=\frac{1}{3} \mathbf{i} \cdot \mathbf{i} \quad \mathbf{P}^{d}=\mathbf{I}-\mathbf{P}^{s p}
$$

Here $I$ is the symmetrie fommth-order identity tensor and $\mathrm{i}$ is the secomd-order identity. 


\subsection{Plasticity}

Plasticity theories were originally developed to mathematically describe permanent deformations in metals which ane associated with dislocation motion and slip at the microstructural level. An important assumption made by these theories is that the lincar clastic properties of the material are not affected by the generation of plastic deformation. This assumption is valid for most motals subjected to only a moderate amomut. of plastic deformation; however, recent experiments indicate that the stiffuess of metals may be affected when large amounts of plastic deformation are generated.

To develop a general framework for plasticity theories, consider the following expression for the strain energy function, ll,

$$
U=\|\left(\epsilon, \epsilon^{\prime \prime}\right)=\frac{1}{2}\left(\epsilon-\epsilon^{\prime \prime}\right): \mathbf{E}:\left(\epsilon-\epsilon^{\prime \prime}\right)
$$

where $\epsilon$ and $\epsilon^{p}$ are the total and plastic strain tensors, respectively. ('olemand and (Burtin (1967) have shown that the ('lausius-1)uhem dissipation inequality can bo written as

$$
\boldsymbol{\sigma}: \dot{\boldsymbol{\epsilon}}-\dot{U} \geq 0
$$

or

$$
\boldsymbol{\sigma}: \dot{\boldsymbol{\epsilon}}-\frac{\partial \boldsymbol{l}}{\partial \boldsymbol{\epsilon}}: \dot{\epsilon}-\frac{\partial l^{\prime}}{\partial \epsilon^{\eta}}: \dot{\epsilon^{\prime}} \geq 0
$$

With the constitutive relation

$$
\boldsymbol{\sigma}=\frac{\partial l}{\partial \epsilon}=\mathbf{E}:\left(\boldsymbol{\epsilon}-\epsilon^{\prime \prime}\right)
$$

and the following expression for the merhanical dissipation rate, $i$,

$$
\dot{D}=-\frac{\partial l}{i \epsilon^{\prime \prime}}: \dot{\epsilon}^{\prime \prime}=: \sigma: \dot{\epsilon}^{\prime \prime}
$$

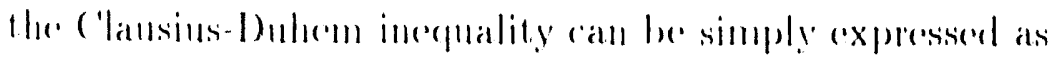

$$
\text { i) } \geq 0
$$

which indieates that the dissipation of mechanical energy is an irreversible process.

Pasticity models are characterized by a yiold function, $\psi$, which definces a surface in stress space separating elastic and plastice regimes and an coolution equation for the plastic strain which is also reforred to as a flow rule. Yiold functions are often written ati follows

$$
\Psi\left(\boldsymbol{\sigma}, \epsilon^{\prime}\right)=g(\boldsymbol{\sigma})-h\left(\epsilon^{\prime \prime}\right)
$$

where $g$ is a function of stress invariants and $h$ is a positive function of $\epsilon^{p}$. Information about the load history is stored in $\epsilon^{p}$ or often some invariant of $\epsilon^{p}$ and the efferes of 


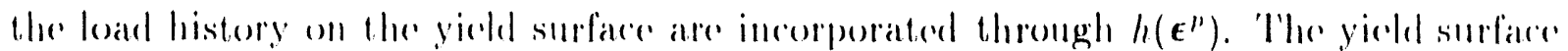

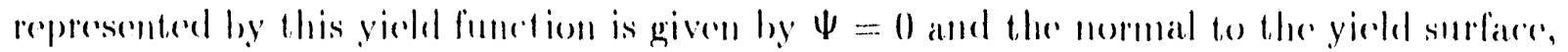
f, is givenll ly

$$
\mathbf{f}=\frac{\partial \psi}{\partial \boldsymbol{\sigma}}
$$

A typical flow rulc is given by

$$
\epsilon^{n}=\dot{\rho g}
$$

where $p$ is a monotonically increasing parameter and $g$ is a seromelorder termsor that represents the orientation of the plastic strain increment. By substituting this expression for the plastie strain rate inte the dissipation rate equation we obtatin

$$
\text { i) }=\dot{\rho} \boldsymbol{\sigma}: \mathbf{g}
$$

For elastic processess, $j$ ceguals zoro and for plastic processes $j$ is positive. Thus, Lymation 3.15 indicates that merhanioal dissipation will be generated when plastie deformation ocenrs and the tonsor product $\sigma: \mathbf{g}$ is pesitive. In other words, te consure that dissipation arcompanies plastic deformation, one should select viold functions and flow laws such that the tensor product $\sigma: \mathbf{g}$ is positive whenever plastic deformation is generated.

The flow rule is refored to as an associated flow rule when the mormal to the yield surface and orientation of the plastice strain increment are c(pual, i.ce, $\mathbf{f}=\mathbf{g}$. 'This condition is also referered to as normality heranse the plastice strain increment is oriented in a direction normal to the yield surfare. Maxill. In plastic dissipation results when associated How rules are used (Simo and Ilughes. 1986). Viedd functions and How rules are not ther-

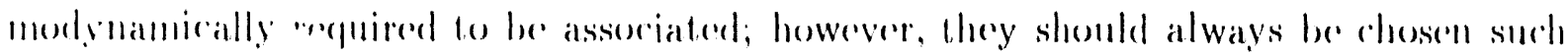
that dissipation and plastic deformation are comedelent events for al! possible loanl paths.

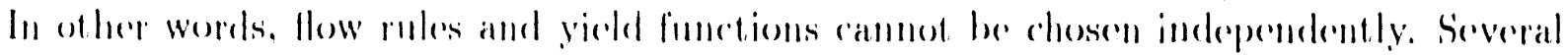

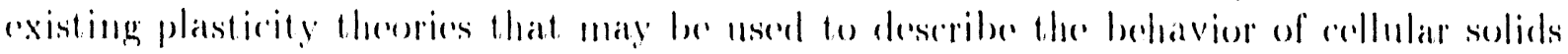
are presented in the mext fow sertions.

\subsubsection{Drucker-Prager}

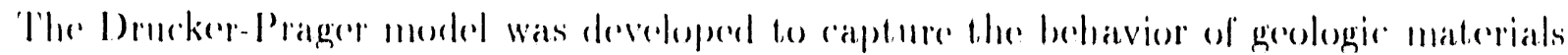
such as sands and soils. This model has a yiold function given by

$$
\psi=\sqrt{I_{2}}+\frac{11}{3} I_{1} \cdots
$$

where $\mu$ is an internal friction parametere and $r$ is a material constant. $I_{2}$ is the secomd invariant of the deviatorice stress terosor and $/ 1$ is the first invariant of the lootal stress torlsor:

$$
I_{2}=\frac{1}{2} \sigma^{\prime l}: \sigma^{d}, \quad l_{1}=\sigma: \mathbf{i}
$$

where $\sigma^{\prime \prime}$ is the stress deviater. The yield surface given by this function is a rone in principal stress spare as shown in ligurere 3.1. 


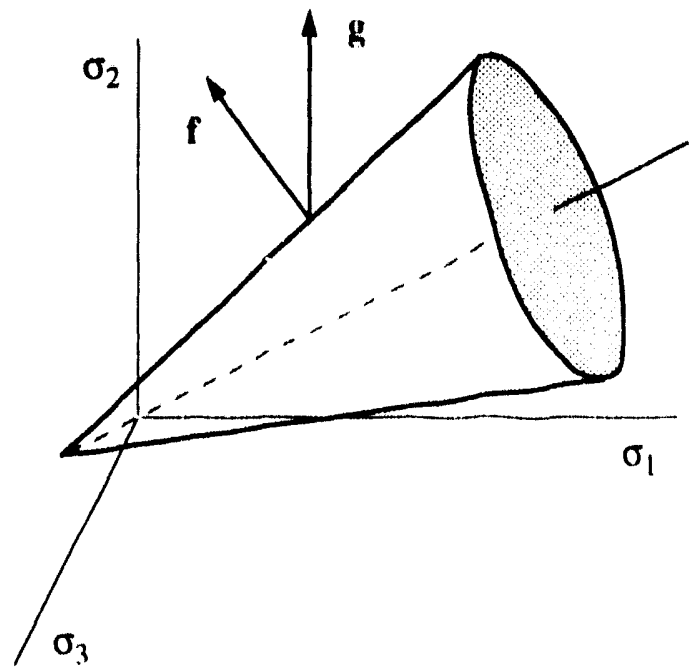

Figure 3.1. Drucker-Prager Yield Sirrace.

The normal lo the yield surface, $f$, is given by

$$
\mathbf{f}=\frac{\boldsymbol{\sigma}^{\prime \prime}}{\left(2 \sqrt{I_{2}}\right)}+\frac{\mu}{3} \mathrm{i}
$$

and the orientation of the plastie strain increment is chosent to be

$$
\mathbf{g}=\frac{\boldsymbol{\sigma}^{\prime \prime}}{\left(2 \sqrt{T_{2}}\right)}+\frac{\beta}{3} \mathrm{i}
$$

where 8 is the dilatancy parameter. When $\mu$ is cepal to 8 the llow is associated with the yield linection.

\subsection{2 von Mises}

Next, consider a von Mises plasticity model with associated flow and lincar strain hardrning. The model was originally developed to deseribe the plastic deformation of metals. The yiold function, $\Psi$, is as follows:

$$
\Psi=\sqrt{I_{2}}-\left(H_{0}+H_{1} \iota^{p}\right)
$$

where $H_{0}$ is the intial yiedd strength, $H_{1}$ is the hardening modulus, and en is the conivalent plastic strain which is a monotonically increasing invariant given by

$$
\epsilon^{\prime \prime}=\int\left(d \epsilon^{p}: d \epsilon^{\mu}\right)^{\frac{1}{2}}
$$




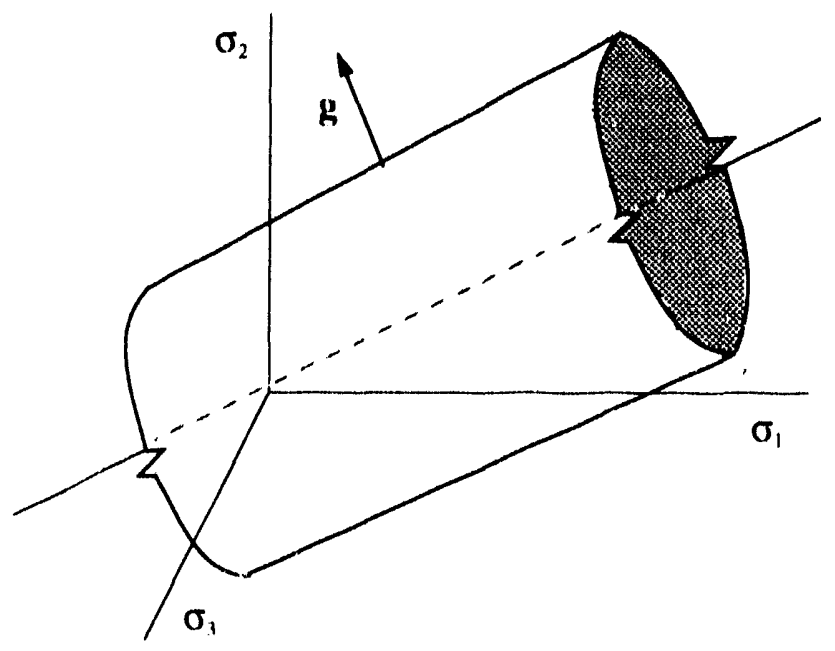

Figure 3.2. von Mises Yield Surface.

This yield function represents a yield surface that is an infinitely long cylinder in stress spare as shown in figure 3.2. The corresponding orientation of the plastic strain increment is normal to the yield surfare and is given by

$$
\mathbf{f}=\mathbf{g}=-\frac{\boldsymbol{\sigma}^{\prime \prime}}{\left(2 \sqrt{J_{2}}\right)}
$$

Equation 3.22 indicates that this model predicts only deviatoric or shape changing plastic strans. Since rigid polyurethane foams and aluminum honeycombs exhibit significant volumetric plastic strains, a von Mises plasticity model is not appropriate for these matrivials.

\subsubsection{Mohr-Coulomb}

Next, consider a simple Molir-('onlomb model which has been used to describe the behavior of sand and other geologic materials. This model has a yield function given by

$$
\Psi=\frac{1}{2}\left(\sigma_{1}-\sigma_{2}\right)+\frac{1}{2}\left(\sigma_{1}+\sigma_{2}\right) \sin \phi-c \cos \phi
$$

where $\phi$ is the internal friction angle, $c$ is the cohesion, and the coordinate system is chosen such that $\sigma_{1}$ and $\sigma_{2}$ are the maximum and minimum principal stresses, respectively. Then for an associated flow law the components of $\mathbf{f}$ and $g$ in the principal coordinate system are

$$
\mathbf{f}=\mathbf{g} \Rightarrow \frac{1}{2}\left[\begin{array}{ccr}
1+\sin \phi & 0 & 0 \\
0 & -1+\sin \phi & 0 \\
0 & 0 & 0
\end{array}\right]
$$

A Tresca model with associated flow is identical to a Mohr-(Coulomb model with the internal friction angle set equal to zero. 


\subsubsection{Principal Stress}

Finally, consider the principal stress theory of Rankine that was recently used by Neilsen, Morgan and Krieg (1987) in a plasticity model for rigid polyurethane foam. This criterion has a yield function given by

$$
\Psi^{i}=\frac{1}{2} \boldsymbol{\sigma}: \mathbf{P}^{i}: \boldsymbol{\sigma}-h\left(\boldsymbol{\epsilon}^{p}\right) \quad i=1,2,3
$$

where $\mathbf{P}^{i}$ is a fourth-order principal projection operator given by

$$
\mathbf{P}^{i}=\mathbf{n}^{i} \otimes \mathbf{n}^{i} \otimes \mathbf{n}^{i} \otimes \mathbf{n}^{i}
$$

and $\mathbf{n}^{i}$ is a unit vector oriented in a principal stress direction. This yield function actually represents six different yield surfaces with normals to the principal stress axes as shown in Figure 3.3.

The flow law is given by

$$
\dot{\epsilon}^{p}=\dot{\rho}^{i} \mathbf{P}^{i}: \boldsymbol{\sigma}
$$

where the repeated indices imply summation. This flow law indicates that plastic strain increments may be associated with each principal stress direction.

Cellular solids generally exhibit both plastic deformation and stiffness changes. Flasticity models predict permanent deformations but assume that the stiffness of the material does not change. Thus, a combination of plasticity theories and continuum damage theories, which predict stiffness changes, are needed for cellular materials. In the next section, continuum damage theories which have a structure similar to existing plasticity theories are presented.

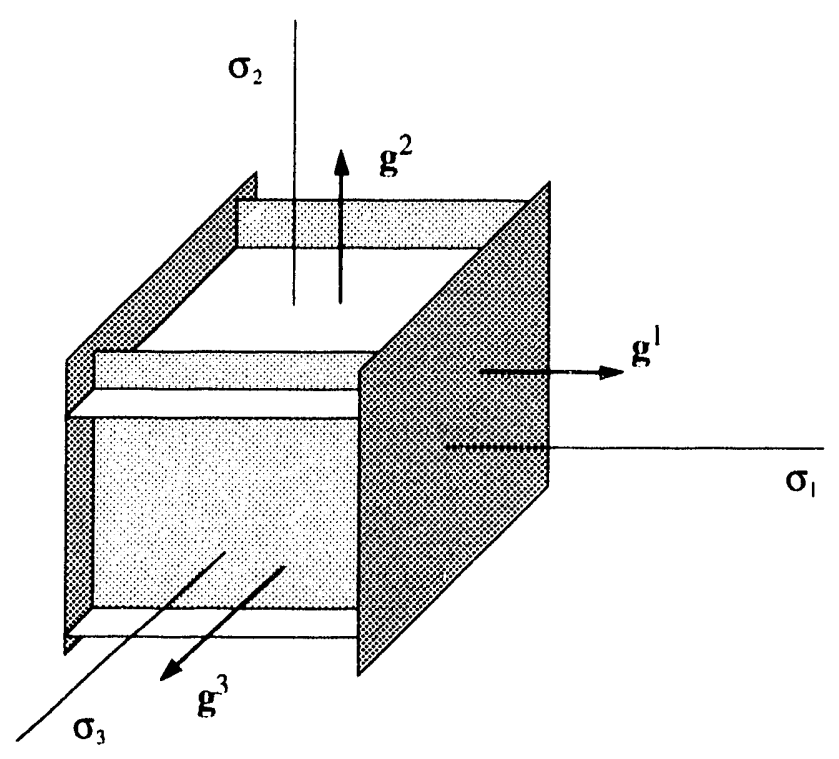

Figure 3.3. Principal Stress Yield Surface. 


\subsection{Continuum Damage}

Continuum damage theories were developed to mathematically describe the progressive reduction in stiffness and strength of brittle materials resulting from the initiation and growth of microcracks and microvoids. These theories have been used to describe the nucleation and growth of microcracks and microvoids in metals and the formation of microcracks in concrete, rocks and ceramics (Kachanov, 1986; Krajcinovic, 1989). An important assumption implicit in these models is that the cracks are small and distributed such that the cracked material can be logically represented by an equivalent contimum with damage. When the cracks are large, fracture mechanics is used to predict the formation and growth of large individual cracks. However, when there are a large number of small cracks it rapidly becomes impossible to follow the formation and growth of cach crack; thus, a continumm damage theory is used.

For the development of continum damage theories, consider the following expression for the strain energy function, $U$,

$$
U=U(\boldsymbol{\epsilon}, \mathbf{S})=\frac{1}{2} \epsilon: \mathbf{S}: \boldsymbol{\epsilon}
$$

where $\mathbf{S}$ is the fourth-order damaged elastic stiffness tensor which is equal to the elasticity tensor, $\mathbf{E}$, when the material is undamaged and changes as the material is damaged. The (lausius-Duhem dissipation inequality can be written as

$$
\boldsymbol{\sigma}: \dot{\boldsymbol{\epsilon}}-\frac{\partial U}{\partial \boldsymbol{\epsilon}}: \dot{\boldsymbol{\epsilon}}-\frac{\partial U}{\partial \mathbf{S}}:: \dot{\mathbf{S}} \geq 0
$$

Using the constitutive relation

$$
\boldsymbol{\sigma}=\frac{\partial U}{\partial \epsilon}=\mathbf{S}: \boldsymbol{\epsilon}
$$

and an expression for the dissipation rate, $\dot{D}$,

$$
\dot{D}=-\frac{\partial U}{\partial \mathbf{S}}:: \dot{\mathbf{S}}=-\frac{1}{2} \epsilon: \dot{\mathbf{S}}: \boldsymbol{\epsilon}
$$

the ('lausius-Duhem inequality is again given by $\dot{D}) \geq 0$. Next, if we introduce an evolution equation for the rate of change in elastic stiffness

$$
\dot{\mathbf{S}}=-\dot{\omega} \mathbf{R}
$$

where $\dot{\omega}$ in some sense represents the increment in damage and $\mathbf{R}$ represents the orientation of the damage increment. The dissipation rate is then given by

$$
\dot{D})=\frac{\dot{\omega}}{2} \epsilon: \mathbf{R}: \epsilon
$$

Using a procedure analogous to that of plasticity, we assume that a damage surface exists and separates elastic and damaging processes. The damage surface is mathematically described by a damage function, $\Psi$,

$$
\Psi=\frac{1}{2} \boldsymbol{\epsilon}: \mathbf{Q}: \boldsymbol{\epsilon}-g(\mathbf{S}, \boldsymbol{\epsilon})
$$


where $\mathbf{Q}$ is some fourth-order tensor and $g$ is some positive function. When $\Psi$ is less than zero the behavior is elastic and when $\Psi$ is equal to zero the behavior may be damaging. As with plasticity, the thermodynamical constraint for dissipation to accompany damage can be satisfied with appropriate selections for the damage function and damage evolution equation. For example, if we use the damage evolution equation given by Equation 3.32 and let $\mathbf{Q}$ equal $\mathbf{R}$ in the damage function, Equation 3.34, then dissipation will always accompany damage.

The damage function given by Equation 3.34 is expressed in terms of strain which implies that the damage function represents a surface in strain space; however, if one is opposed to working in strain space the damage function can always be expressed in terms of stress. For example, choose

$$
\mathbf{Q}=\mathbf{S}: \mathbf{P}: \mathbf{S}
$$

then

$$
\Psi=\frac{1}{2} \boldsymbol{\sigma}: \mathbf{P}: \boldsymbol{\sigma}-g(\mathbf{S}, \boldsymbol{\epsilon})
$$

We conld also use an evolution equation for the compliance of the material, C, in plare of the evolution equation for the stiffuess given by Equation 3.32. Since: $\mathbf{C}: \mathbf{S}=\mathbf{I}$, the evolution equations for stiffuess and compliance must be related as follows

$$
\dot{\mathbf{C}}=-\mathbf{C}: \dot{\mathbf{S}}: \mathbf{C}
$$

Evolution equations which would be appropriate for the damage function given by Equation 3.36 are

$$
\dot{\mathbf{S}}=-\dot{\omega} \mathbf{S}: \mathbf{P}: \mathbf{S} \quad \text { or } \quad \dot{\mathbf{C}}=\dot{\omega} \mathbf{P}
$$

Thus, for damage functions in stress space it may be easier to use revolution equations for compliance. At this point, we have placed no restrictions on the forms for $\mathbf{Q}$ and $\mathbf{R}$ and have simply stated that they should be chosen such that the thermodymamical restriction for dissipation to arcompany damage is satisfied.

\subsubsection{Simple Scalar Damage}

Must existing contiuum damage models are scalar damage models. For these models the orientation of the damage is given by some fixed fourth-order tensor. (One of the simplest scalar damage models has a damage function given by

$$
\Psi=\frac{1}{2} \boldsymbol{\epsilon}: \mathbf{E}: \boldsymbol{\epsilon}-h(\mathbf{S}, \boldsymbol{\epsilon})
$$

and an evolution equation for the elastic stiffness tensor given by

$$
\dot{\mathbf{S}}=-\dot{\omega} \mathbf{E}
$$

where $\mathbf{E}$ is the isotropic elastic stiffness tensor given by Equation 3.3. This evolution equation indicates that the damage orientation is given by the original undamaged elastic 
stiffness tensor. With this evolution equation, the current elastic stiffness tensor, $\mathbf{S}$, is related to the undamaged elastic stiffuess tensor, $\mathbf{E}$, as follows

$$
\mathbf{S}=(1-\omega) \mathbf{E}
$$

Since the elastic stiffuess tensor, $\mathbf{E}$, is isotropic, the damaged elastic stiffness tensor, $\mathbf{S}$, is also isotropic. As the material is damaged, the elastic modulus is degraded as follows

$$
E=(1-\omega) E^{\circ}
$$

where $E$ and $E^{\circ}$ are the elastic moduli for the damaged and original undanaged material, respectively. With this model, information about the current damaged state is stored in a single scalar, $E$. This continum damage model is quite simple; however, the appropriateness of this model for any damaging materials has not been demonstrated because Equation 3.41 implies that Poisson's ratio remains unchanged.

\subsection{2 von Mises Damage}

Next consider a scalar damage model which has the same yield function as a von Mises plasticity model written as follows

$$
\Psi=\frac{1}{2} \boldsymbol{\sigma}: \mathbf{P}^{d}: \boldsymbol{\sigma}-g^{2}(\mathbf{S})
$$

On the surface, this yield function looks different than the yield function given by Equation 3.20. However, both Equation 3.20 and this equation represent a yield surface that is an infinitely long cylinder in principal stress space as shown in Figure 3.2. An appropriate damage evolution equation for this model is given by

$$
\dot{\mathbf{S}}=-\dot{\omega} \mathbf{S}: \mathbf{P}^{d}: \mathbf{S}=-4 \dot{\omega}\left(i^{2} \mathbf{P}^{d}\right.
$$

where (i represents the damaged shear modulus for the material. For this model the damage history is stored in the single scalar ( $i$. A von Mises plasticity model is characterized by deviatoric plastic strains and no change in the volumetric response of the material. Likewise, a von Mises damage model is characterized by reductions in the shear stiffness of the material and no change in the volumetric stiffness of the material.

\subsubsection{Principal Stress Damage}

Next, consider the principal stress criterion of Rankine which has damage functions given by

$$
\Psi^{i}=\frac{1}{2} \boldsymbol{\sigma}: \mathbf{P}^{i}: \boldsymbol{\sigma}-h(\mathbf{S}) \quad i=1,2,3
$$

where $\mathbf{P}^{i}$ is again the fourth-order principal projection operator. The damage evolution equations for this model are chosen to be

$$
\dot{\mathbf{S}}=-\dot{\omega}^{i} \mathbf{S}: \mathbf{P}^{i}: \mathbf{S} \quad \text { or } \quad \dot{\mathbf{C}}=\dot{\omega}^{i} \mathbf{P}^{i}
$$


where the repeated indices imply smmmation. This model is not a simple scalar damage model and information about damage is not stored in a single scalar becanse the orientation of the damage, specifically, $\mathbf{P}^{i}$, can change during a loading process. For this model, information about the current damaged state is stored in the damaged clastic stiffness tensor, $\mathbf{S}$, or the damaged compliance tensor, $\mathbf{C}$.

\subsubsection{Principal Strain Damage}

Finally, consider a principal strain criterion which has recently been used by Bazant and Lin (1988) to describe the behavior of concrete. This model is able to predict the formation of vertical cracks in concrete specimens subjected to miaxial compression. This model has damage functions given by

$$
\Psi^{i}=\frac{1}{2} \boldsymbol{\epsilon}: \mathbf{P}^{i}: \boldsymbol{\epsilon}-h(\mathbf{S}) \quad i=1,2,3
$$

where $\mathbf{P}^{i}$ is again the fourth-order principal projection operator; however, now the $\mathbf{n}^{i}$ used in the definition of $\mathbf{P}^{i}$ in Equation 3.26 are vectors oriented in a principal strain direction. Bazant and Lin's (1988) crack hand model uses a damage evolution equation given by

$$
\dot{\mathbf{C}}=\dot{\omega}^{i} \mathbf{P}^{i}
$$

The interesting characteristic of this model is that for certain load paths this model will predict damage without dissipation. For example, under uniaxial compression lateral tensile strains are generated and damage is predicted but the dissipation equation

$$
\dot{D}=\dot{\omega}^{i} \frac{1}{2} \boldsymbol{\sigma}: \mathbf{P}^{i}: \boldsymbol{\sigma}
$$

indicates that no dissipation is generated. This model cond be improved by simply using a different damage evolution equation. For example, if one of the following damage evolntion equations is used with the damage function given by Equation 3.47, then dissipation will acrompany damage for all load paths.

$$
\dot{\mathbf{S}}=-\dot{\omega}^{i} \mathbf{P}^{i} \quad \text { or } \quad \dot{\mathbf{C}}=\dot{\omega}^{i} \mathbf{C}: \mathbf{P}^{i}: \mathbf{C}
$$

This brief review of principal strain models clearly shows that evolution equations and damage functions cannot be selected arbitrarily. The examples in this section show that damage theories which are analogous to plasticity theories can be developed using a similar framework. In the next section, we explore theories in which continumm damage is compled with plasticity. 


\subsection{Coupled Theories}

If both damage and plastic deformations are generated then the developmenteste presented in the previons sertions most be modified to apture the compling. The strain energy liutrelion is now given by

$$
l=l\left(\epsilon, \epsilon^{\prime \prime}, \mathbf{S}\right)=\frac{1}{2}\left(\boldsymbol{\epsilon}-\epsilon^{\prime \prime}\right): \mathbf{S}:\left(\boldsymbol{\epsilon}-\epsilon^{\prime \prime}\right)
$$

The ('lansius-1)uheme imeguality heromess

$$
\boldsymbol{\sigma}: \dot{\epsilon}-\frac{\partial l}{\partial \epsilon}: \dot{\epsilon}-\frac{\partial l !}{\partial \epsilon^{\prime \prime}}: \epsilon^{\prime}-\frac{\partial U l}{\partial \boldsymbol{S}}:: \dot{\mathbf{S}} \geq 0
$$

'Plue constitutive relation is given by

$$
\sigma=\frac{\partial I}{\partial \epsilon}
$$

and the dissipation rate by

$$
\dot{D}=-\frac{\partial l l}{\partial \mathbf{S}}:: \dot{\mathbf{S}}-\frac{\partial \| l}{\partial \boldsymbol{\epsilon}^{n}}: \dot{\epsilon}^{n}=-\frac{1}{2}\left(\boldsymbol{\epsilon}-\boldsymbol{\epsilon}^{\prime \prime}\right): \dot{\mathbf{S}}:\left(\boldsymbol{\epsilon}-\boldsymbol{\epsilon}^{p}\right)+\boldsymbol{\sigma}: \dot{\epsilon}^{\prime \prime}
$$

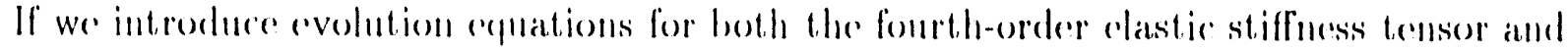
the serond-order platstie strain ternsor as follows:

$$
\dot{\mathbf{S}}=-\dot{w} \mathbf{R} \quad \dot{\boldsymbol{\epsilon}}^{\prime \prime}=\dot{\rho} \mathbf{g}
$$

then the dissipation rate is given by

$$
\dot{l})=\dot{\rho} \boldsymbol{\sigma}: \mathbf{g}+\frac{\dot{w}}{2}\left(\boldsymbol{\epsilon}-\boldsymbol{\epsilon}^{p}\right): \mathbf{R}:\left(\boldsymbol{\epsilon}-\boldsymbol{\epsilon}^{p}\right)
$$

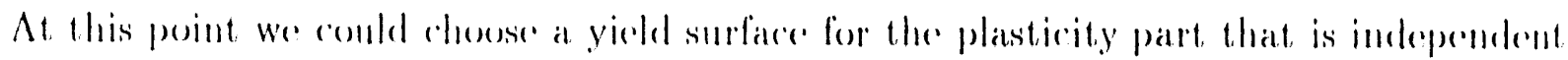
from the damage surfare and reguire that both torms in Equation 3.56 satisly the dissipation inceguality. 'This wonld cortainly he a sulficiont condition. However for many materials, it serems reasomahle to experet that damage will only he generated when peremancent strains are generated which implies that for such materials the damber and yield surfaces are the same. Even with this assmmption, there is still a signiticant antombt of latitude in rhosing appropriate covolution equations for permanent strain and damage.

For example, if we chocose a yichl function given by

$$
\Psi=\boldsymbol{\sigma}: \mathbf{P}: \boldsymbol{\sigma}-g\left(\boldsymbol{\epsilon}, \boldsymbol{\epsilon}^{n}, \mathbf{S}\right)
$$

where $g$ is some pessitive function, and a damage evolution equation and flow law given by

$$
\dot{\mathbf{S}}=-\dot{\omega} \mathbf{S}: \mathbf{P}: \mathbf{S} \quad \dot{\boldsymbol{\epsilon}}^{\prime \prime}=\dot{\rho} \mathbf{P}: \boldsymbol{\sigma}
$$


then the dissipation rate is given by

$$
\dot{D}=(\dot{\rho}+\dot{\omega}) \boldsymbol{\sigma}: \mathbf{P}: \boldsymbol{\sigma}
$$

Use of the yedd function given by Equation 3.57 and the evolution equations given hy Equation 3.58 will ensure that mechanical dissipation is generated whenever damage or plastic deformation is gencrated. An interesting feature of this compled theory is that o and $\omega$ are not reguired to monotonically increase as long as the stum of the two terthis does. What this means is that stiffuess of the material conld actually be increasing whilo plastic deformation is being generated and the theory would still he thermodynamically acceptable. The requirement that damage monotonically increases is only applicable to damage only theories; however, for most materials that exhihit both damage and permanent strain it may still be appropriate based on physical arguments to assmme that damage is not a reversible process even though the assmmption of irrerersibility is not required.

To complete this compled theory we need an additional equation to determine the relative amounts of damage and plastic strain generated during a given step. For example, wo rould postulate a relationship of the form

$$
M \dot{p}=(1-M) \dot{\omega}
$$

where $M$ is some material parameter ohtained from experimental olsenvations. When $M$ is equal to zero the theory represents a plasticity only theory since $\dot{\omega}$ is apllal to zero and when $M$ is expal to one, the theory reduces to a damage only theory. When $M$ is between zere and one both permanent strains and damage are generated when llae material is loaded inte the inelastic regime.

\subsubsection{Simple Scalar Damage Coupled with Plasticity}

Most damage models are simple isotropie, scalat damage models. One of the simplest

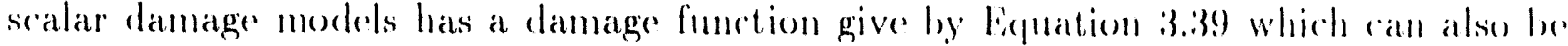
written as

$$
\Psi=\boldsymbol{\sigma}: \mathbf{C}: \boldsymbol{\sigma}-\eta\left(\mathbf{S}, \boldsymbol{\epsilon}, \boldsymbol{\epsilon}^{\prime \prime}\right)
$$

where $\mathrm{C}$ is the compliance tensor and is equal to the inverse of the damaged elatsticity tensor, S. For this model, the evolution equations for plastic strain and damage are given by

$$
\dot{\epsilon}^{\prime \prime}=\dot{\rho} \mathbf{C}: \boldsymbol{\sigma}=\dot{\rho}\left(\boldsymbol{\epsilon}-\boldsymbol{\epsilon}^{p}\right) \quad \dot{\mathbf{S}}=-\dot{\omega} \mathbf{E}
$$

where the relationship between $\dot{\rho}$ and $\dot{\omega}$ is given by Equation 3.6it). With this morlel, the orientation of the plastic strain increment is given by the chastic strain tensor, $e^{2}=$ $\left(\epsilon-\epsilon^{p}\right)$. 


\subsection{2 von Mises}

Next consider a coupled von Mises theory with a elanage (yiedd) function given by

$$
\Psi=\frac{1}{2} \boldsymbol{\sigma}: \mathbf{P}^{d}: \boldsymbol{\sigma}-g^{2}\left(\epsilon^{p}\right)
$$

The flow rule and damage evolution equation are given by

$$
\dot{\epsilon}^{\prime \prime}=\dot{\rho} \mathbf{P}^{d l}: \boldsymbol{\sigma} \quad \dot{\mathbf{E}}=-\dot{\omega} 4 c^{\prime 2} \mathbf{P}^{d}
$$

As experted, this compled model is chatacterized by deviatoric plastic strains, reductions in the shear stiffuess, and a linear elastic volmmetrir response.

\subsubsection{Principal Stress}

Finally, consider a principal stress rriterion of Rankine that was recently used in a plasticity model for polyurethane foams by Neilsen, Morgan and Krieg (1987). If wo wanted to develop a compled model based on this same criterion we womld use a dambage (yield) function given by

$$
\Psi^{\prime}=\frac{1}{2} \boldsymbol{\sigma}: \mathbf{P}^{i}: \boldsymbol{\sigma}-h\left(\boldsymbol{\epsilon}, \boldsymbol{\epsilon}^{p}, \mathbf{S}\right)
$$

where $\mathbf{P}^{1}$ is again the fonth-order principal projection operator. One possible choice for the plastic and damage flow rules would be

$$
\dot{\epsilon}^{\prime \prime}=\dot{\rho}^{i} \mathbf{P}^{i}: \boldsymbol{\sigma} \quad \dot{\mathbf{S}}=-\dot{\omega}^{\prime} \mathbf{S}: \mathbf{P}^{i}: \mathbf{S}
$$

These examples show that coupled theories ran be easily developed using the general framework presented in this section. 


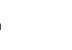




\section{BIFURCATIONS AND LOCALIZATION}

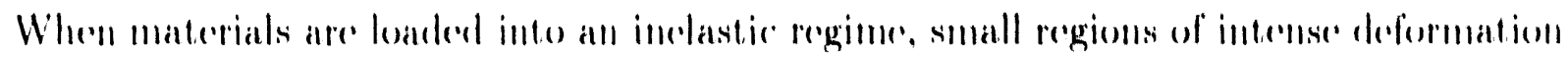
often develop at somse point in the loading process. This inlense localized deformation

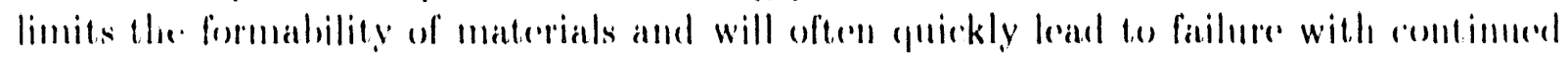
lodeling. Localization is often associaled with satisfaction of the classical discontinumens

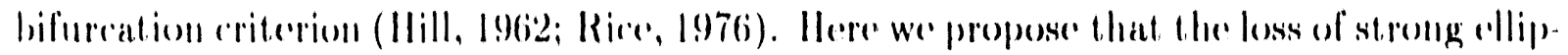

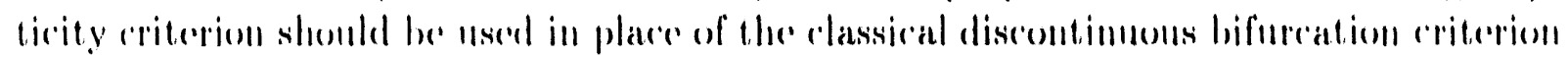
as a mecessary condition for localization. The application of the strong elliplicity reriterion impleses that a hifureation mode assoriated with loss of positive dedindereness of the

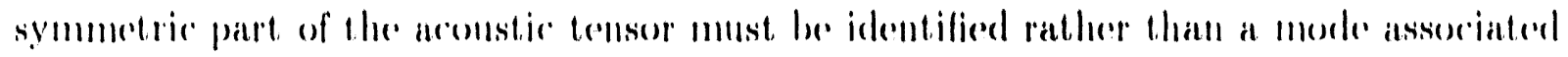

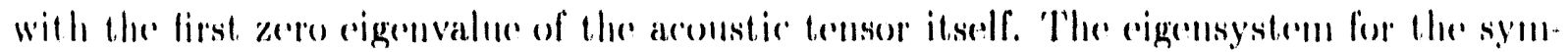
metrie part of the tangent stiffess terisor is obtained for several different plasticity allol rontimumm damage models. This rigensystem provides information about deformation modes associated with hoth diffuse and discontimuons hifureations. Malerial properties, bommelary romditions, and hody geometry are all shown to affere the difluse and localized deformation modes that are generated. Numerons experimental observations of merking and localization in metal specimens sulp joet to varions bonndary conditions are explaimed with a hifureation analysis of a von Mises plasticity model. Finally, hifurcation andelyses are performed on constitutive theories which may he appropriate for collular soliels.

\subsection{Introduction to Bifurcation Criteria}

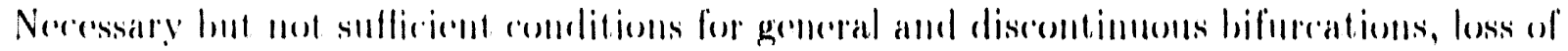
moicqueness, and localized deformation of elasticeplastic materials have heren previously developed. A meressary comdition for loss of material stability, loss of muigurencess and a general hifureation in the solution is the loss of positive definiteness of the rate of serend

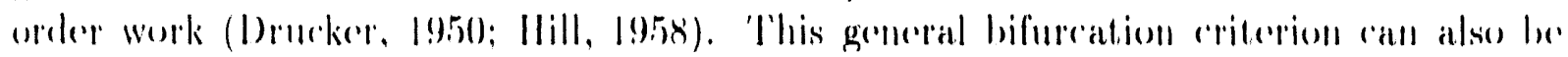
expresserel as loss of pesitive definiteness of the symmetrie part of the tangent stifluess

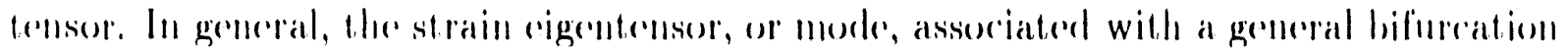
will sot have a kimematically compatible form. Thus, the mode can only exist in a zome

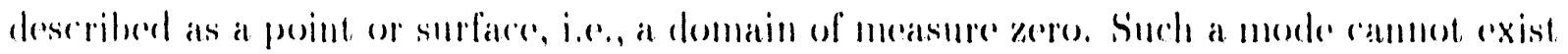
in any real sellse without affereting the stress state in the surromeding material and is usually referend to as a diffuse mode which initiates smooth changes in the deformation firld such as merking.

Valanis (1989) states that loss of material stability shomld be associated with the limit point where the tangent stiffuess tensor obtains a zero eigenvalue. This womld be appropriate if onc considers only statically determinate specimens with force prescribed 


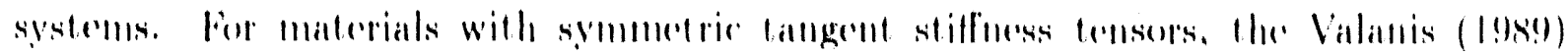

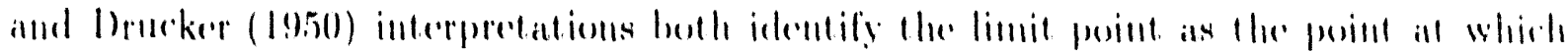

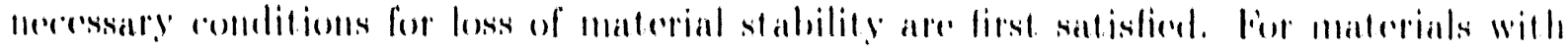

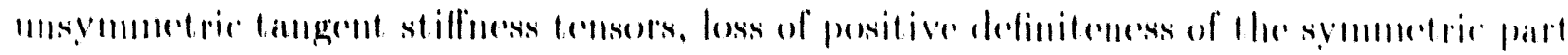

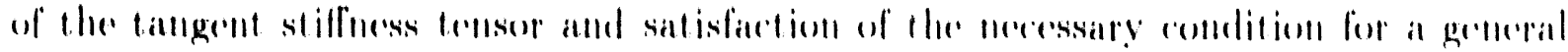

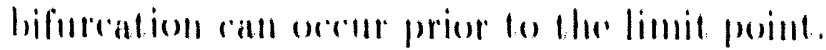

Ilill (19662), Mandel (1966(i), and Rudnicki and Rice (1975) have suggestod that loss of

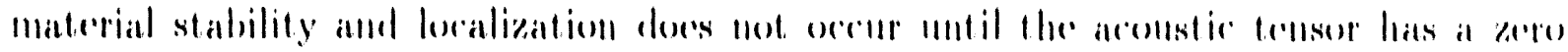

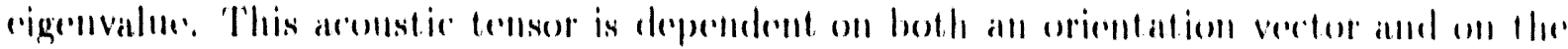

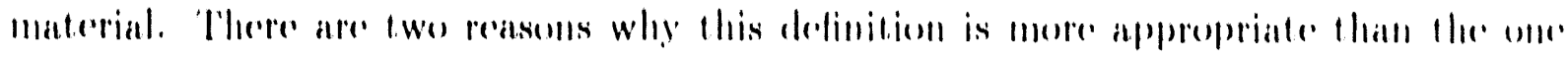

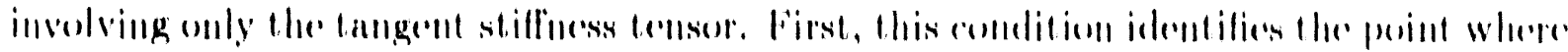

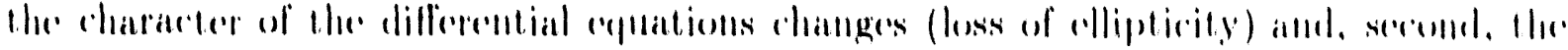

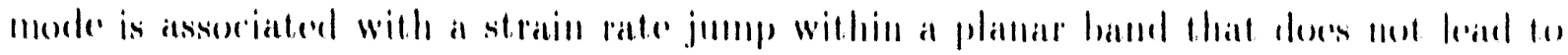

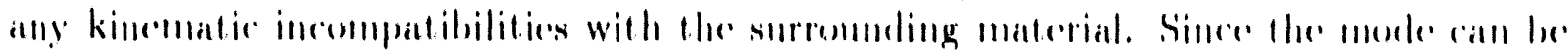

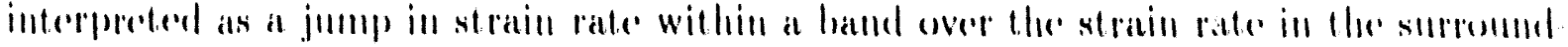

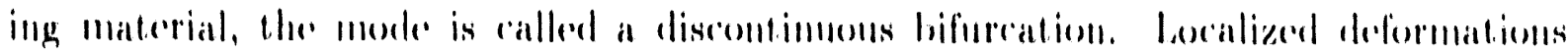

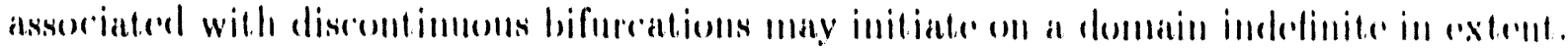

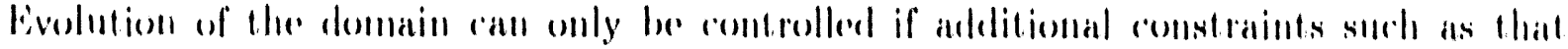

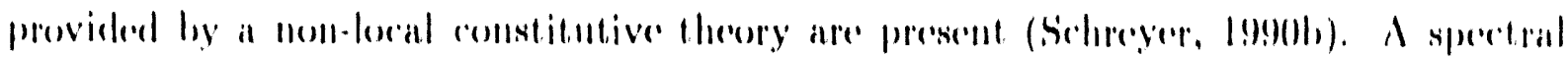

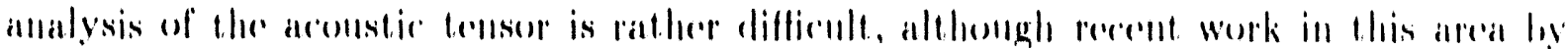

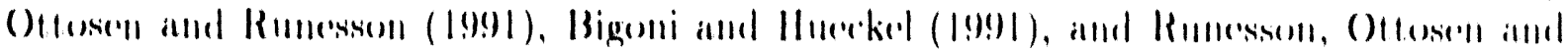

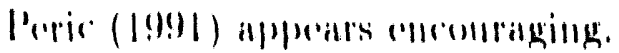

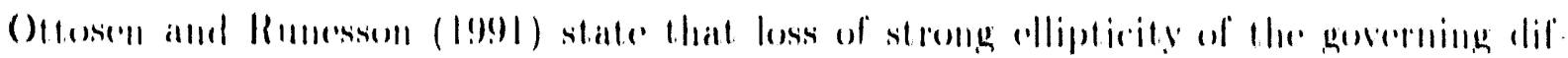

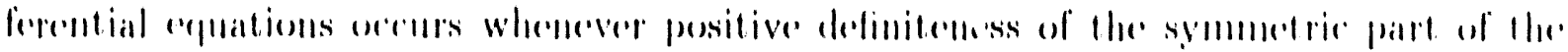

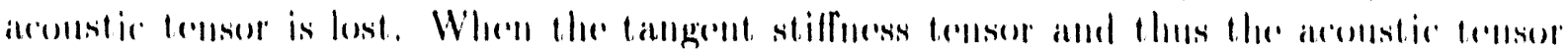

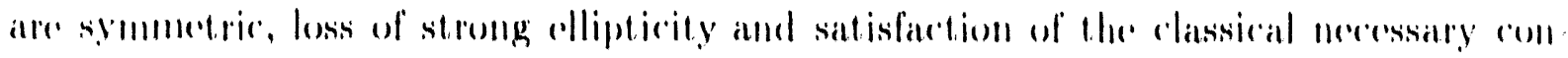

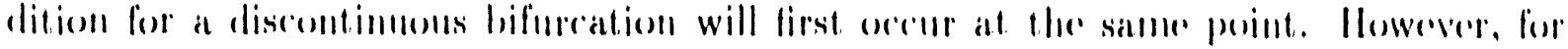

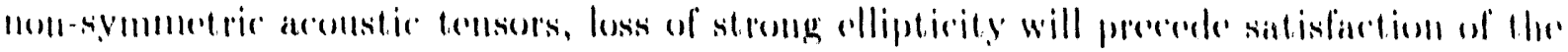

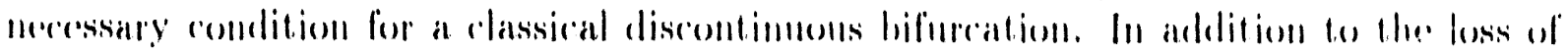

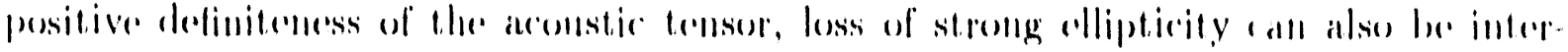

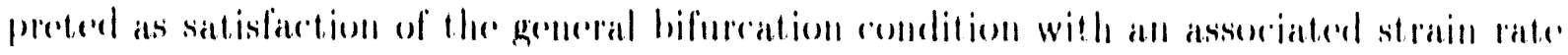

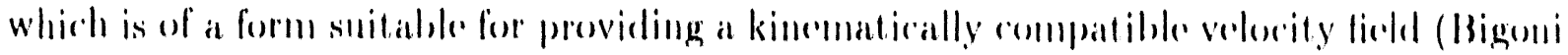

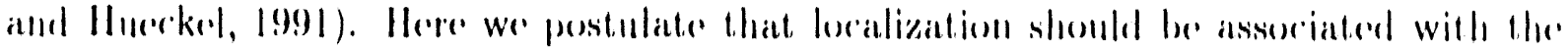
loss of strong cllipticity.

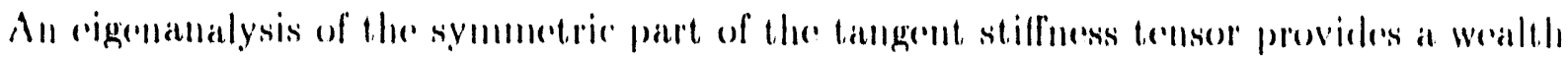
of information alont deformation modes associated with both diffuse and discontinnons bifureations. The meressary enolition for a goneral bifincation is first satisfied when the fundamental eigenvalue of the symmetrie part of the tangent stiffuess teresof olutains a

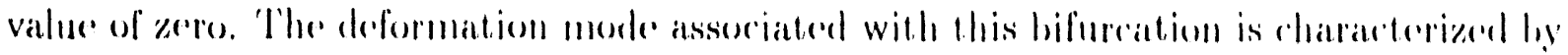

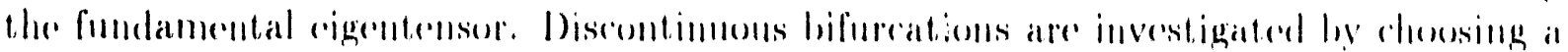
mode which satisties the gerderal hifureation criterion and is restrieterd to be of a form 
mormally assoriaterd with a discomtimuons bifurcation. The mode is expressed as a lin. car comblination of the regentensors associaterl with the symmetric part of the langent. stiffuess ternsor. With this approach there is no need to explicitly determine or analyze the acoustic tonsor. By interpreting the problem of material stability as an eigensystem probleme in the presenese of a ronstraint, we antomatically establish the strueture for in. corporating additional constraints, such as plane strain, which may be present beranse of extermal londing and bomedary conditions. The presence of additional constraints antes. matically infer that the uswal procedure of finding the first acro eigenvalue of the aroustic tensor may not be an indicator of loralization.

The approach used is to determine the spectral deromposition of the symmetrie part of the tangent stiffuess terosor. For conventional astir-plastic models this decomposition is straightforward and explicil lincar combinations of rigentensors can be obtained to show satisfaction of ronstraint: which reduce lo linear algebraic equations of the rigentensors. Some of the constraints can only be satisfied if the fumblamental eigenvaluse is megative which only ocrurs when plasticity morlels with associated flow rules exhibit strain soft. ening. 'The degeres of softening, if any, recpuired to meet the constraint condition deponds oil the particular loaling path bering considered.

With the insight provided hy this approach, we show that features exhibited hy a mumber

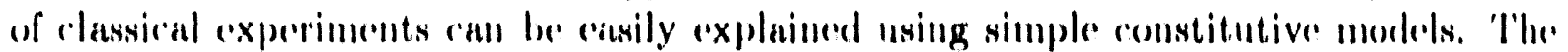
failure monles of a material subjere to varions constraints can provide valuable confirma. tion of the sultability of a constitutive model. For simplicity, we confine ond attention to rale and temporature inderendent material behavior and infinitewimal deformations.

\subsubsection{General Bifurcations}

Drucker (1950, 1959) postulated that the stability of a material could he evaluated by considering the work done by an external agenry. A material is stable (will remain in (efuilibrimm) if a) positive work is (lome by the external agency during the application of the seet of stressess and h) the lleet work done hy it over a cycle of application and removal is acro or positive. If plastic deformation is generated during the cycte then the net work must be non-zero. 'These statements indicate that a necessary condition for loss of material stability is

$$
\dot{\boldsymbol{\epsilon}}: \dot{\boldsymbol{\sigma}}=0
$$

where $\dot{\sigma}$ and $\dot{e}$ are perturbations to the stress and strain rate fields at some point or region in the body dere to the external agency. Equation 4.1 was shown by Hill (1958) to

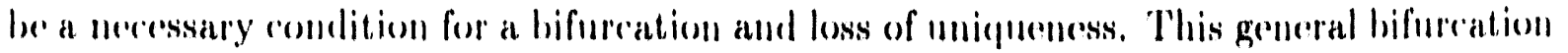
criteriom, Eyuation 1.1 , call also be written as

$$
\dot{\boldsymbol{\epsilon}}: \mathrm{D}^{*}: \dot{\boldsymbol{\epsilon}}=\mathbf{0}
$$

where $\mathrm{D}^{s}$ is the symmontric part of the tangent stiffuess tensor, $\left.\left.\left.l\right)_{i, k l}^{s}=\frac{1}{2}(l)_{i, k l}+l\right)_{k \cdot 1,1}\right)$.

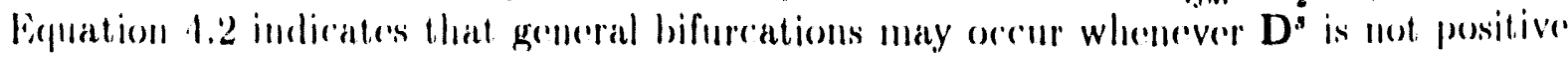
definite. 


\subsubsection{Limit Point Bifurcations}

(iemeral bifurcations are desually associated with rhanges in the stress state both inside

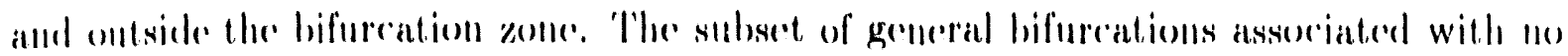
rlange in the stress state ocent only at the linit point when

$$
\mathrm{D}: \dot{\epsilon}=0 \quad \text { or } \quad \text { de }(\mathrm{D})=1)
$$

or, in where words, when the tangent stiffuess ternsor D has a zero eigenvalue. Valanis (1989) recontly suggested that Equation 4.3 is a necessary and sufticient condition for loss of material stability. His interpretation assmomes that the general bifureation associated with the limit point is always activated. This interpretation is only valid when the tangent stiffuess tensor is symmetrie and tractions are preseribed everywhere on the homolary of a specimen. If constraints are present, the general bifureation associated with the limit point may not be activated, and it becomes necessary to evaluate other potential general and discontimuons bifurcations. Here we adopt the rassical necessary condition for a general hifureation given by Equation 4.2.

\subsubsection{Classical Discontinuous Bifurcations}

('riteria for discontimuons bifurentions in alastir-plastir materials with assoriated How rules follow from Hadamard's (19)(13) studies of elastic stability and were developoed hy

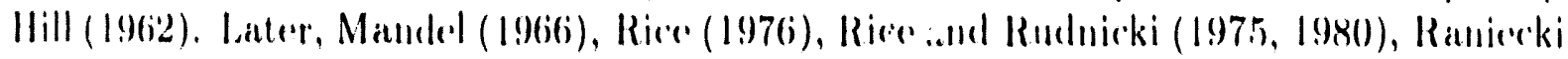

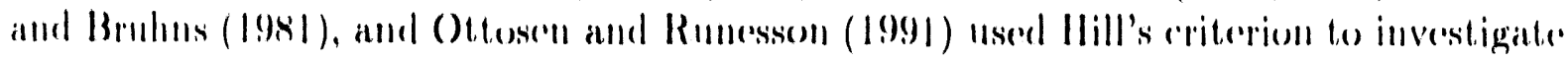
discontimuons lifurcations in clastic-plastic materials with non-associated How rules.

('onsider a homogeneons solid subjerted to monotonice, proportional loading. Wo wish to determine al what point in the loading process a discontinnoms bifurcation can orecur surb that sulsergurent strain rates berome discontinuous across parallel planes of orientation n that separate a zone of localized deformation from the rest of the borly. Maxwell's compatibility conditions require that the strain rate in the localized zonse, $\dot{\epsilon}^{\prime}$, he of the for'll

$$
\dot{\boldsymbol{\epsilon}}^{\prime}=\dot{\boldsymbol{\epsilon}}^{\prime \prime}+\dot{\boldsymbol{\epsilon}}^{k} \quad \text { wilh } \quad \dot{\boldsymbol{\epsilon}}^{k}=\frac{1}{2}(\mathbf{m}(0) \mathbf{n}+\mathbf{n}(\cdot) \mathbf{m})
$$

where $\dot{\epsilon}^{\prime \prime}$ is the strain rate ontside the localizand zone, $\dot{\epsilon}^{k}$ is a kimematioally admissible discontinnous mode, and $m$ can be interporeted as a vector that represents the orientation of the relative velocity of regions on opposite sides of the localized deformation zone dure to the introduction of the localized deformation.

Assume that the entire body is being plastically deformed, the stress and strain components are uniform throughout, and the body is at the onset of localization. With the assumption of rate independent behavior, the stress rates inside and ontside the localized zone are given by

$$
\dot{\boldsymbol{\sigma}}^{i}=\mathbf{D}^{\prime}: \dot{\boldsymbol{\epsilon}}^{\prime} \quad \text { and } \quad \dot{\boldsymbol{\sigma}}^{\prime \prime}=\mathbf{D}^{\prime \prime}: \dot{\boldsymbol{\epsilon}}^{\prime \prime}
$$


where D' and D" are the tangent stilluess tensons for material inside and ontside the

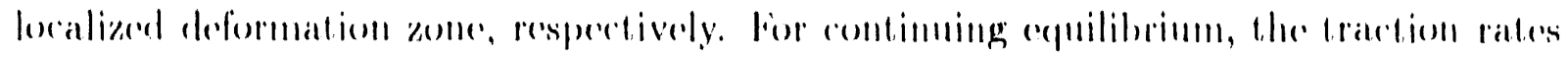

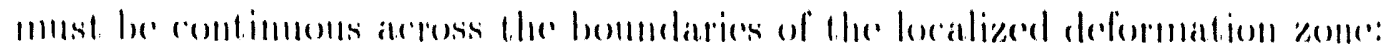

$$
\dot{\mathrm{t}}^{\prime}-\dot{\mathrm{t}}^{\prime \prime} \quad \text { or } \quad \mathrm{n} \cdot\left(\dot{\boldsymbol{\sigma}}^{\prime}-\dot{\boldsymbol{\sigma}}^{\prime \prime}\right)=\mathbf{0}
$$

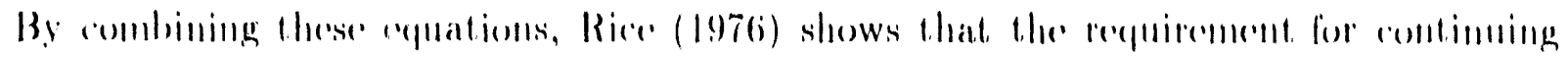
apuilibrinum is given l,y

$$
n \cdot\left(D^{\prime}-D^{\prime \prime}\right): \dot{c}^{\prime \prime}+\mathbf{Q} \cdot \mathbf{m}=\mathbf{0}
$$

where

$$
\mathbf{Q}=n \cdot \mathbf{D}^{\prime} \cdot \mathbf{n}
$$

is the aroustic terlisor.

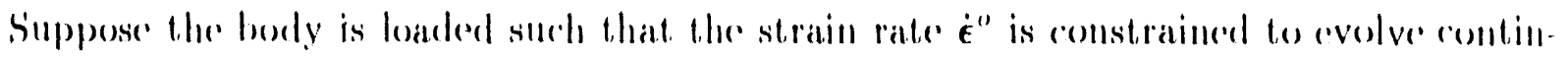
monsly. Then it is reasomable to assume that the langent stiffuess teresor for material ontside the localized zome, D", is identieal to the tangent stillness terusor for material inside the localized zone, D', al the intiation of the bifureation. The classieal necessatry romelition for a discontimusess hifureation is then obtained from Fequation 4.7:

$$
\mathbf{Q} \cdot m=0 \quad \text { or } \quad \operatorname{del}(\mathbf{Q})=0
$$

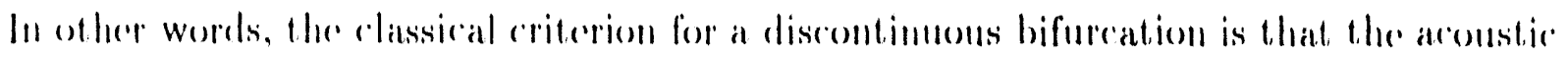

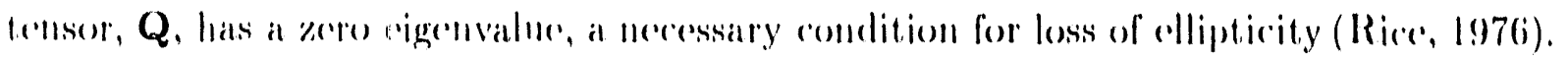

\subsubsection{Loss of Strong Ellipticity}

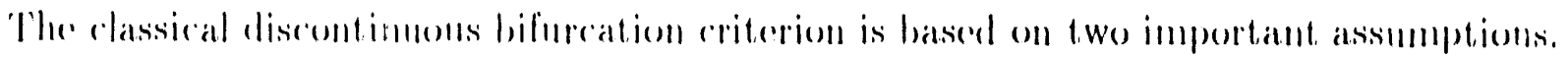

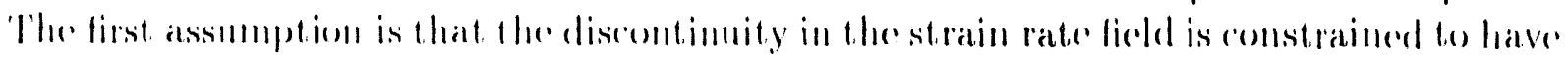

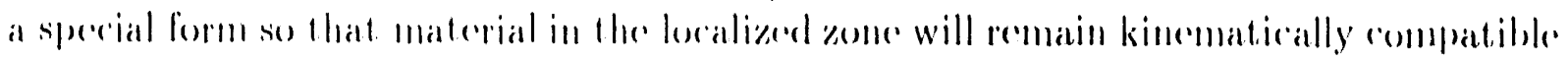
with the surroumeling material. The serend assmomption is that the strain rates covelve

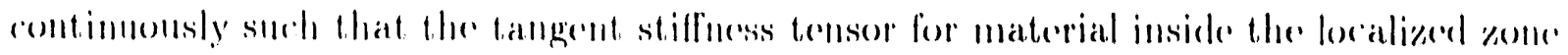

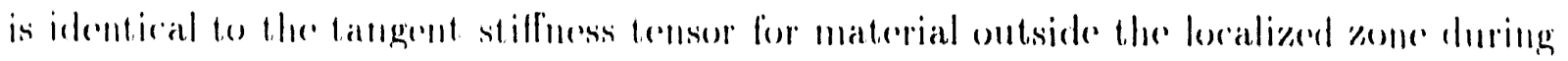

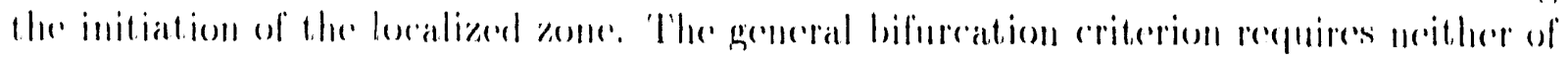
these assumptions. Siperilically, a general hifureation will not meressarily he associalend with a mode which has the sperial lorm of $\dot{\epsilon}^{k}$ in begmation 4.4 and the andive tangent stiffuess tensors for matereal inside and ontside the hifureation zone will not neressarily le identicial.

The general bifuration criterion, bequation 4.2, is a mecessary condition for any type of hifuration. A mecessary comdition for a general bifureation with a kincomalioally compatible moder, $\dot{\epsilon}^{k}$, is the loss of strong cellipticity criterion (Bigoni and Ilucelkel, 1991)

$$
\dot{\boldsymbol{\epsilon}}^{k}: \mathbf{D}^{*}: \dot{\boldsymbol{\epsilon}}^{k}=0 \quad \Rightarrow \quad \mathrm{m} \cdot \mathrm{Q}^{*} \cdot \mathbf{m}=0 \text {. }
$$


We adopt loss of strong ellipticity as a necessary condition for localization because this criterion identifies the first possible bifurcation with a kinematically compatible mode.

The requirement for continuing equilibrium, Equation 4.7, may be satisfied when loss of strong ellipticity occurs if the continuity constraint (in time) on $\dot{\boldsymbol{\epsilon}}^{o}$ is relaxed. For example, let $\mathbf{Q}$ be decomposed into its symmetric, $\mathbf{Q}^{s}$, and antisymmetric, $\mathbf{Q}^{u}$, parts:

$$
\mathbf{Q}=\mathbf{Q}^{s}+\mathbf{Q}^{u}
$$

Loss of strong ellipticity, Equation 4.10, will first occur when

$$
\mathbf{Q}^{s} \cdot \mathbf{m}=\mathbf{0} \quad \text { or } \quad \operatorname{det}\left(\mathbf{Q}^{s}\right)=0
$$

since $\mathbf{Q}^{s}$ is positive, semi-definite at this point. This criterion, Equation 4.12 , will be satisfied prior to or at the same time as the classical discontinuous bifurcation criterion of Equation 4.9. If no external constraint is placed on $\dot{\epsilon}^{\circ}$ other than compatibility, then $\dot{\boldsymbol{\epsilon}}^{o}$ is free to adjust such that the continuing equilibrium equation

$$
\mathbf{n} \cdot\left(\mathbf{D}^{i}-\mathbf{D}^{o}\right): \dot{\boldsymbol{\epsilon}}^{o}+\mathbf{Q}^{u} \cdot \mathbf{m}=\mathbf{0}
$$

is satisfied when the loss of strong ellipticity criterion is satisfied. This means that a discontinuous bifurcation may occur when the loss of strong ellipticity criterion is satisfied and $\dot{\boldsymbol{\epsilon}}^{\circ}$ is not constrained.

\subsubsection{Summary of Bifurcation Criteria}

The criteria for diffuse and discontinuous bifurcations are summarized in Table 4.1. The general bifurcation criterion is first satisfied when the determinant of the symmetric part of the tangent stiffness tensor is equal to zero. For materials with associated flow, the tangent stiffness tensor is symmetric and the general and limit point bifurcation criteria buth identify the limit point as the first point at which a diffuse bifurcation may occur. However, for maierials with non-associated flow the general bifurcation criterion indicates that diffuse bifurcations may occur in the hardening regime.

Table 4.1. Summary of Bifurcation Criteria.

\begin{tabular}{|c|c|c|}
\hline CRITERIA & EQUATION & MODE \\
\hline Cieneral & $\dot{\boldsymbol{\epsilon}}: \mathbf{D}^{s}: \dot{\boldsymbol{\epsilon}}=0$ & Diffuse, Necking \\
Limit Point & $\mathbf{D}: \dot{\boldsymbol{\epsilon}}=\mathbf{0}$ & Diffuse, Necking \\
Strong Ellipticity & $\mathbf{m} \cdot \mathbf{Q}^{s} \cdot \mathbf{m}=0$ & Localized \\
Ciassical Discontinuous & $\mathbf{Q} \cdot \mathbf{m}=\mathbf{0}$ & Localized \\
\hline
\end{tabular}

The strong ellipticity criterion is first satisfied when the determinant of the symmetric part of the acoustic tensor is equal to zero. For materials with associated flow rules, the strong ellipticity and classical discontinuous bifurcation criteria identify the same 
first discontinuous bifurcation point. However, for materials with non-associated flow the strong ellipticity criterion will predict that localization may occur prior to the point identified by the classical discontinuous bifurcation criterion.

Discontinuous bifurcations are a subset of general bifurcations and classical discontinuous bifurcations are a subset of those discontinuous bifurcations which satisfy the loss of strong ellipticity criterion. Constraints may inhibit the activation of certain possible bifurcation modes for which the nccessary but not sufficient conditions given in the previous section have been satisfied.

\subsection{Characterization of Bifurcation Modes}

Bifurcation modes, $\dot{\boldsymbol{\epsilon}}$, represent perturbations to a homogeneous strain rate field that may be activated whenever the necessary conditions presented in the previous section are satisfied. Any bifurcation mode, $\dot{\boldsymbol{\epsilon}}$, can be characterized by its three eigenvalues, $\lambda_{1} \leq \lambda_{2} \leq \lambda_{3}$. Modes associated with discontinuous bifurcations are restricted to be of the kinematically compatible form, $\dot{\boldsymbol{\epsilon}}^{k}$, given in Equation 4.4. Suppose we choose a local coordinate system with coordinate $x_{1}$ parallel and coordinates $x_{2}$ and $x_{3}$ perpendicular to $\mathbf{n}$ such that the components of $\mathbf{n}$ and $\mathbf{m}$ are

$$
\mathbf{n} \Rightarrow\left\{\begin{array}{l}
1 \\
0 \\
0
\end{array}\right\} \quad \mathbf{m} \Rightarrow\left\{\begin{array}{c}
\alpha \\
2 \beta \\
0
\end{array}\right\}
$$

The components of the corresponding discontinuous bifurcation mode, $\dot{\boldsymbol{\epsilon}}^{k}$, are

$$
\dot{\epsilon} \Rightarrow\left[\begin{array}{lll}
\alpha & \beta & 0 \\
\beta & 0 & 0 \\
0 & 0 & 0
\end{array}\right]
$$

When $\beta$ is equal to zero, the relative velocity of the bodies is oriented in a direction normal to the zone, and the strain rate jump in the zone represents an opening mode (Figure 4.1). When $\alpha$ is equal to zero, the strain rate jump represents a shearing mode. Also, note that $\dot{\epsilon}$ has eigenvalues of $\alpha / 2 \pm \sqrt{\alpha^{2} / 4+\beta^{2}}$ and zero. Thus, a discontinuous bifurcation mode has a fundamental eigenvalue, $\lambda_{1}$, that is less than or equal to zero, an intermediate eigenvalue, $\lambda_{2}$, that is equal to zero, and a third eigenvalue, $\lambda_{3}$, that is greater than or equal to zero.

Modes associated with general bifurcations can be any symmetric second-order tensor as long as the necessary condition for a general bifurcation, Equation 4.2 , is satisfied. For example, a general bifurcation mode could have components obtained as a slight generalization of Equation 4.15:

$$
\dot{\boldsymbol{\epsilon}} \Rightarrow\left[\begin{array}{lll}
\alpha & \beta & 0 \\
\beta & 0 & 0 \\
0 & 0 & \rho
\end{array}\right]
$$




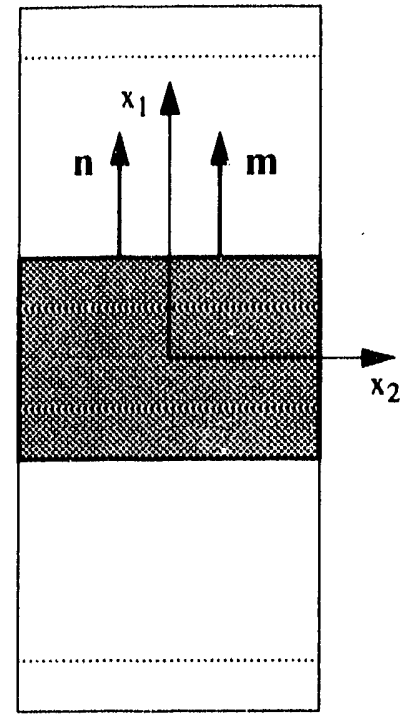

(a) opening mode

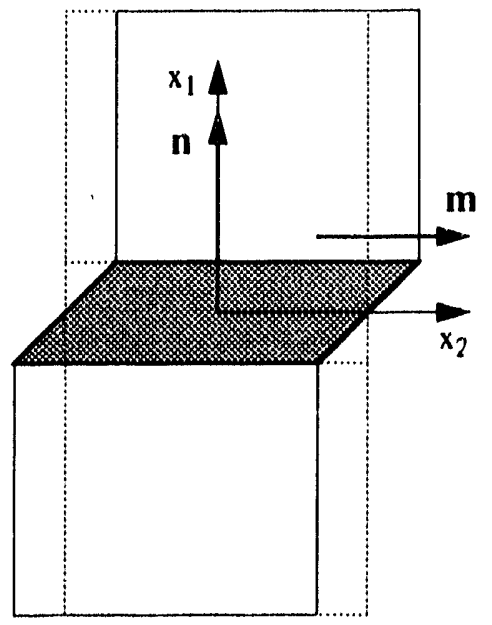

(b) shearing mode

Figure 4.1. Discontinnous Bifurcation Modes.
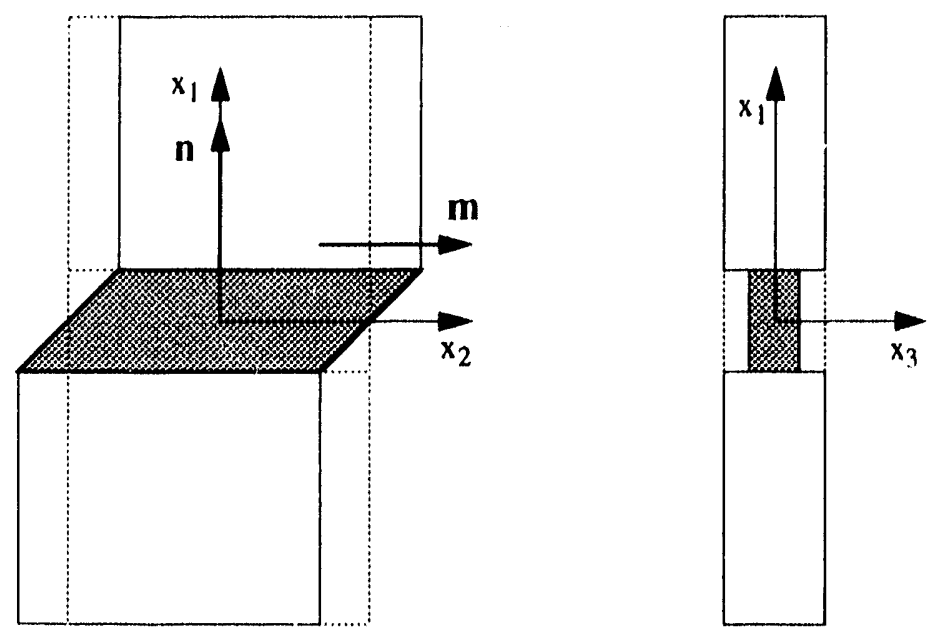

Figure 4.2. General Bifurcation Mode. 
An analysis in the $x_{1}-x_{2}$ plane can be performed as before for a discontinuous bifurcation with the origin of $x_{3}$ at the surface of a possible discontinnity; however, now an incompatibility in the velocity field exists for points $x_{3} \neq 0$ which are not in the $x_{1}-r_{2}$ plane (Figure 4.2). There are two ways to interpret this situation. In brittle materials, some experimental specimens exhibit microcracking in a specific orientation which cond be considered a manifestation of the incompatible velocity field. In ductile materials, the potential development of an incompatible mode will lead to changes in the local stress state and the evolution of smooth changes in the deformation field such as necking.

The construction given above displays a compatible mode in the $x_{1}-x_{2}$ plane with the possibility of an incompatible component in the $x_{3}$ direction. Of course, a compatible mode could possibly exist in other planes. The actual orientation of the necked region might be based on the geometry of the specimen. For example, if the dimensions of a specimen in the $x_{1}$ and $x_{2}$ directions are much larger than the dimension in the $x_{3}$ direction, then there might be a preference for most materials to neck with the direction of the potential incompatibility oriented towards the minimum dimension of the specimen.

In general, for a localized zone to form and remain compatible in some plane with the surrounding material, there must be some orientation in the plane such that the normal component of the localized mode is equal to zero. In terms of the eigenvalues associated with a bifurcation mode, compatibility can only exist in principal planes for which $\lambda_{i} \lambda_{i} \leq$ 0 . Recall, that a discontinuous bifurcation mode has a zero intermediate principal value and is thus compatible in all three principal planes. Ceneral bifurcation modes with one positive and two negative eigenvalues are compatible only in the principal planes with eigenvalues of opposite sign. Finally, general bifurcation modes that are positive or negative definite are compatible only at a single point and are not compatible in any principal plane.

\subsection{Bifurcation Criteria and the Eigensystem for $\mathbf{D}^{s}$}

In this section, we investigate the relationship between the bifurcation criteria presenter in the previous section and the eigensystem associated with the symmetric part of the tangent stiffness tensor, $\mathbf{D}^{s}$. Consider the eigenvalue problem

$$
\mathbf{D}^{s}: \mathbf{x}_{i}=\omega_{i} \mathbf{x}_{i}
$$

in which $\mathbf{x}_{i}$ denotes an eigentensor for $\mathbf{D}^{s}$ and $\omega_{i}$ the corresponding eigenvalue. Due to its minor symmetries, $\mathbf{D}^{s}$ has six symmetric and three skew-symmetric eigentensors. Sinco the bifurcation modes, $\dot{\epsilon}$, are symmetric second-order tensors, we confine onr attention to only the symmetric eigentensors associated with $\mathbf{D}^{s}$ and refer to them simply as the eigentensors associated with $\mathbf{D}^{s}$ throughout the remainder of this paper. For convenience, we normalize the eigentensors so that

$$
\mathbf{x}_{i}: \mathbf{x}_{j}=\delta_{i, j}
$$


and order the eigenvalues such that $\omega_{1} \leq \omega_{2} \leq \ldots \omega_{61}$. 'The strain rate perturbation, or any symmetric second-order tensor, can be written as a lincar combination of the cigentensors associated with $\mathbf{D}^{s}$ as follows:

$$
\dot{\boldsymbol{\epsilon}}=\sum_{i=1}^{(i} \alpha_{i} \mathbf{x}_{i}
$$

and the necessary condition for a general bifuration from Equation 4.2 an be writien as

$$
\dot{\boldsymbol{\epsilon}}: \mathbf{D}^{s}: \dot{\boldsymbol{\epsilon}}=\sum_{i=1}^{6} \gamma_{i}^{2} \omega_{t}=0
$$

The necessary condition for a general bifurcation is first satisfied when $\omega_{1}=0$, and the corresponding bifurcation mode is characterized by the fundamental eigentensor.

If constraints from the geometry or boundary conditions are present, the bifurcation more given by the fundamental eigentensor may not be artivated. If the material strain softens with continned loading, the fundamental eigenvalue will become negative and the necessary condition, Equation 4.20, for mumerous other diffuse and discontinuons bifurcation modes will be satisfied. A discontinuons bifurcation associated with loss of strong ellipticity may be activated when the general hifurcation criterion is satisfied and the corresponding $\dot{\epsilon}$ has a special form. Specifically, the intermediate eigenvalue for this second-order tensor, $\dot{\epsilon}$, must equal zero. Finally, localization will only occur when the necessary condition for a discontinuous bifurcation mode that is not constrained by the boundary conditions is satisfied.

\subsection{Eigenanalysis of the Elastic Tangent Stiffness Tensor}

Eigenanalyses of the tangent stiffness tensors provide a wealth of information abont both diffuse and discontinuons bifurration modes and aid in the identification of constraiued bifurcation modes. Here, it is shown that the cigensystem for an clastic tangent stifluess tensor can be casily obtained. In subseguent sections, eigensystems for tangent stillness tensors associated with plastic and damaging processes are obtained.

For an olastic increment in an isotropic material, the tangent stiffuess tensor is equal to the elasticity tensor, $\mathbf{E}$, which is repeated here for convenience:

$$
\mathbf{E}=3 K \mathbf{P}^{s p}+2\left(i \mathbf{P}^{d}\right.
$$

where $k$ is the bulk modulus and $(B$ is the shear modulus for the clastic material. 'Tlue bulk and shear moduli are related to Young's modulus, E, and Poisson's ratio, r, as follows:

$$
K=\frac{E}{3(1-2 v)} \quad\left(i=\frac{E}{2(1+v)}\right.
$$


The fourth-order spherical projection operator, $\mathbf{P}^{s p}$, and the deviatoric projection operator, $\mathbf{P}^{d}$, are given by

$$
\mathbf{P}^{s p}=\frac{1}{3} \mathrm{i} \infty \mathrm{i} \quad \mathbf{P}^{d}=\mathbf{I}-\mathbf{P}^{s p}
$$

Here $\mathbf{I}$ is the symmetric fourth-order identity tensor and $\mathrm{i}$ is the second-order identity. The spherical projection operator, $\mathbf{P}^{s p}$, has only one nonzero eigenvalue of one with a corresponding eigentensor equal to the second-order identity. All of the other eigentensors for $\mathbf{P}^{s p}$ are in a deviatoric space, a space of symmetric second-order tensors orthogonal to $\mathbf{i}$. The deviatoric projection operator, $\mathbf{P}^{d}$, has an eigenvalue of one with a multiplicity of five. The corresponding five eigentensors are orthogonal to $\mathbf{i}$ and span the deviatoric space. The second-order identity is also an eigentensor for $\mathbf{P}^{d}$ with a corresponding eigenvalue of zero. With this information and the expression for $\mathbf{E}$, one observes that $\mathbf{E}$ has an eigenvalue of $3 K$ with a multiplicity of one and an eigenvalue of $2 C$ with a multiplicity of five. The corresponding eigentensors are the second-order identity and any set of five tensors which are orthogonal to the identity and span the deviatoric space, respectively. Specifically, the components in a Cartesian coordinate system of the normalized eigentensors, $\mathbf{e}_{i}$, for $\mathbf{E}$ can be chosen to be the following:

$$
\frac{1}{\sqrt{6}}\left[\begin{array}{ccc}
-1 & 0 & 0 \\
0 & 2 & 0 \\
0 & 0 & -1
\end{array}\right], \frac{1}{\sqrt{2}}\left[\begin{array}{ccc}
-1 & 0 & 0 \\
0 & 0 & 0 \\
0 & 0 & 1
\end{array}\right], \frac{1}{\sqrt{2}}\left[\begin{array}{lll}
0 & 1 & 0 \\
1 & 0 & 0 \\
0 & 0 & 0
\end{array}\right], \frac{1}{\sqrt{2}}\left[\begin{array}{lll}
0 & 0 & 1 \\
0 & 0 & 0 \\
1 & 0 & 0
\end{array}\right], \frac{1}{\sqrt{2}}\left[\begin{array}{lll}
0 & 0 & 0 \\
0 & 0 & 1 \\
0 & 1 & 0
\end{array}\right], \frac{1}{\sqrt{3}}\left[\begin{array}{lll}
1 & 0 & 0 \\
0 & 1 & 0 \\
0 & 0 & 1
\end{array}\right]
$$

with corresponding eigenvalues of $\lambda_{i}=2\left(i\right.$ for $i=1,5$ and $\lambda_{6}=3 k$. The set of eigentensors given above is not unique; however, the set must always span the space of symmetric second-order tensors. Also, note that if Poisson's ratio equals zero then $2(i=3 k=E$ and all of the eigenvalues are equal to $E$ and $\mathbf{E}=E \mathbf{I}$. Since, the elastic: tangent stiffness tensor, $\mathbf{E}$, is symmetric and positive definite, bifurcations cannot occur during elastic loading or unloading.

\subsection{Eigenanalysis of the Plastic Tangent Stiffness Tensor}

A plasticity model is characterized by a yield function, $\Psi$, which defines a surface in stress space separating elastic and plastic regimes and a flow rule

$$
\dot{\epsilon}^{p}=\dot{\rho} \mathbf{g}
$$

where $\mathbf{g}$ is a second-order tensor which defines the orientation of the plastic strain increment and $\rho$ is a monotonically increasing parameter. The tangent stiffness tensor for a plastic increment is given by

$$
\mathbf{D}=\mathbf{E}-\frac{1}{A} \mathbf{E}: \mathbf{g} \otimes \mathbf{f}: \mathbf{E}
$$


where $f$ is the normal to the yield surfare defined by the yiedd function w:

$$
f=\frac{\partial \Psi}{\partial \sigma}
$$

The sralat $A$ is given by

$$
A=\|+\mathbf{g}: \mathbf{E}: \mathbf{f}
$$

where $/ 1$ is the generalized strain hardening modulus given by

$$
\|=\frac{\partial \psi}{\partial \epsilon^{\prime \prime}}: \dot{\epsilon^{\prime \prime}}
$$

which is pesitive, zero, or megative for strain hardening, perfect, and strain soltening plasticity, respectively. Pastic loanding occurs when $\Psi=0$ and $\mathbf{f}: \mathbf{E}: \dot{\boldsymbol{\epsilon}}>0$.

('onsider the rigensystems of $\mathbf{E}:\left(\lambda_{i}, \mathbf{e}_{i}\right)$ and $\mathbf{D}^{s}:\left(\omega_{i}, \mathbf{x}_{i}\right)$ as presented previously. 'Tho clastic tangent stiffuess tensor, $\mathbf{E}$, is symmetric and positive definite. Also, the cigenvertors for $\mathbf{E}$ span the space of symmetric second-order teensors. The eigenvalues and cigenvertors for the symmetric part of the plastic tangent stiffiess tensor, D", will dopend on the specitic plasticity model being used. Since, the eigentensors of $\mathbf{E}$ span the spare of symmetrie second-order tensors, we can express $f$ and $g$ ats a lincar combination

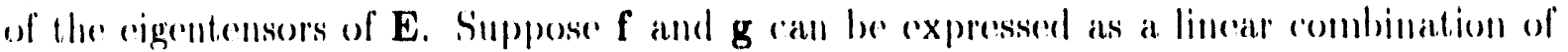
t.wo of the elastic rigentensors (say the first and serond to be spereitic). 'Then

$$
\mathbf{f}=f_{1} \mathbf{e}_{1}+f_{2} \mathbf{e}_{2} \quad \mathbf{g}=g_{1} \mathbf{e}_{1}+y_{2} \mathbf{e}_{2}
$$

andil

$$
\mathbf{E}: \mathbf{f}=l_{1} \lambda_{1} \mathbf{e}_{1}+l_{2} \lambda_{2} \mathbf{e}_{2} \quad \mathbf{E}: \mathbf{g}=g_{1} \lambda_{1} \mathbf{e}_{1}+l_{2} \lambda_{2} \mathbf{e}_{2}
$$

Pestulate an erigentensor for $\mathrm{D}^{s}$ of the form

$$
\mathbf{x}=\xi_{1} \mathbf{e}_{1}+\xi_{2} \mathbf{e}_{2}
$$

Then

$$
\mathbf{D}^{s}: \mathbf{x}=\mathbf{E}: \mathbf{x}-\frac{\mathbf{f}: \mathbf{E}: \mathbf{x}}{2 A} \mathbf{E}: \mathbf{g}-\frac{\mathbf{g}: \mathbf{E}: \mathbf{x}}{2 A} \mathbf{E}: \mathbf{f}=\omega\left(\xi_{1} \mathbf{e}_{1}+\xi_{2} \mathbf{e}_{2}\right)
$$

which shows that the postulated form of Equation 4.32 is valid. After aguating the corllicionts of e and $e_{2}$, the result is

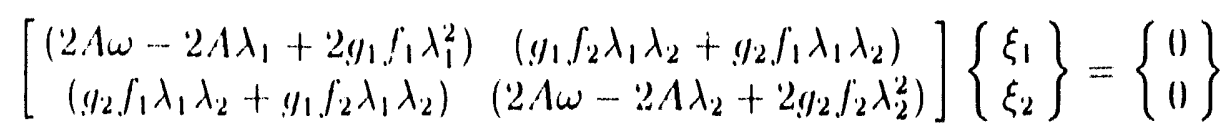

For a nontrivial solution to exist, the determinant of the coeflicient matrix must equal zere. The quadratic characteristic equation yields the two eigenvalues $\omega_{1}, \omega_{2}$ and the corresponding normalized cigentensors $x_{1}$ and $x_{2}$ are obtained by determining the assos-

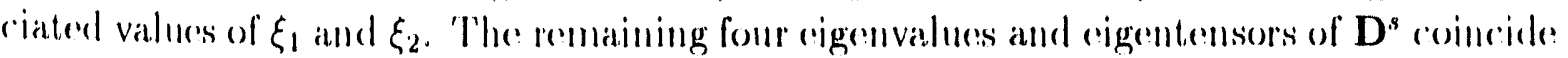
with these of $\mathbf{E}$ and span the remaining space of symmetric second-order tensors. 'Tlhe procedure can be extenderl in a similar manner to the case where $f$ and $g$ arr members of any sulsspace of the space spanmed by the eigentensors of $\mathbf{E}$. 


\subsection{Bifurcation Analysis of Plasticity Models}

In this seretion, hifincation andyses are performed on several existing plasticity montels to characterige the natere of the bifureations predieted hy these models and determine

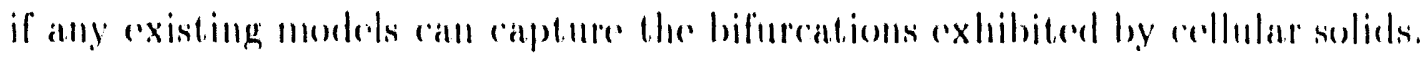

\subsubsection{Drucker-Prager}

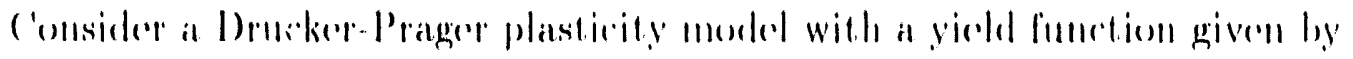

$$
\psi=\sqrt{I_{2}}+\frac{\mu}{3} l_{1}-k
$$

where $I_{2}$ is the serond invariant of the deviatorie steresses and $/ 1$ is the first invariant of the total stress terisot as follows:

$$
l_{2}=\frac{1}{2} \sigma^{\prime \prime}: \sigma^{\prime \prime}, \quad l_{1}=\boldsymbol{\sigma}: \mathbf{i}
$$

where $\sigma^{\prime \prime}$ is the stress deviator. The normal to the yiceld surfare, $f$ is given hy

$$
f=\frac{\sigma^{\prime \prime}}{\left(2 \sqrt{T_{2}}\right)}+\frac{\mu}{3} \mathrm{i}
$$

and the orientation of the phastire strain increment, is

$$
\mathbf{g}=\frac{\boldsymbol{\sigma}^{\prime \prime}}{\left(2 \sqrt{T_{2}}\right)}+\frac{\not 3}{3} \mathbf{i}
$$

The tangent stilfuess tonsor for a plastic step is given by Equation 4.26.

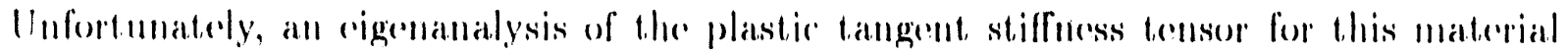

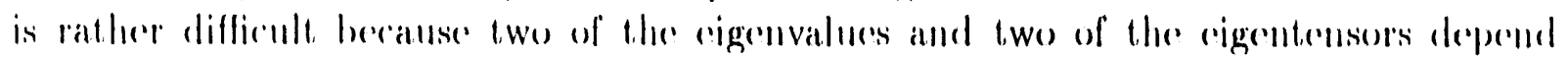

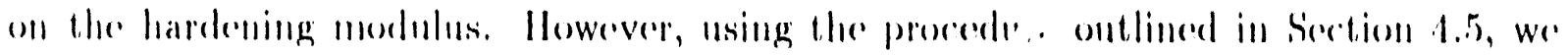

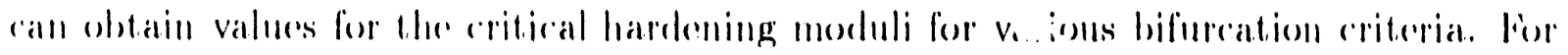
any loading, the terosors $f$ and $g$ can be written as a lincar combination of t.wo of the

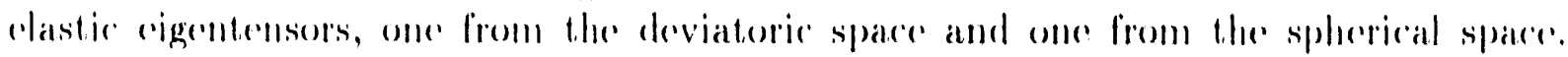

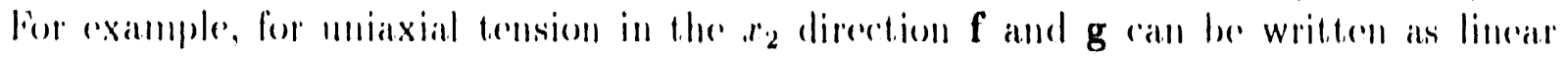
rombinations of $\mathbf{e}_{1}$ and $\mathbf{e}_{t}$ from byuation 4.24 as follows:

$$
\mathbf{f}=f_{1} \mathbf{e}_{1}+f_{6 i} \mathbf{e}_{6 i} \quad \mathbf{g}=l_{1} \mathbf{e}_{1}+g_{6} \mathbf{e}_{6}
$$

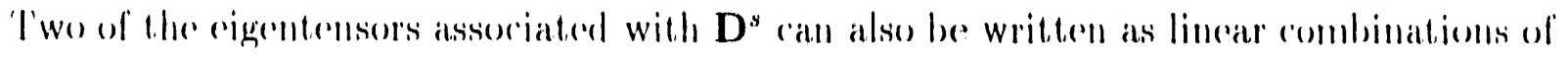
$\mathbf{e}_{1}$ and $\mathbf{e}_{6}$ as follows:

$$
\mathbf{x}_{1}=\gamma_{1} \mathbf{e}_{1}+\gamma_{6} \mathbf{e}_{6 i} \quad \mathbf{x}_{6}=\rho_{1} \mathbf{e}_{1}+\rho_{0 i} \mathbf{e}_{6 i}
$$

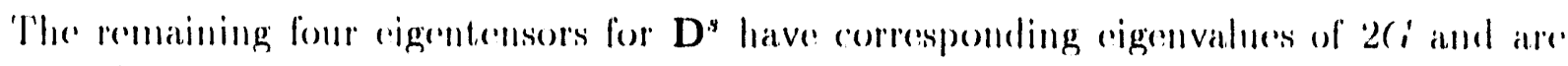
given by

$$
\mathbf{x}_{\mathbf{\imath}}=\mathbf{e}_{i} \quad i=2,3,4,5
$$




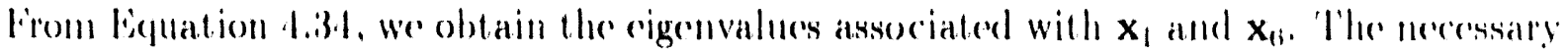
condition for a general bifureation is first satisfied when the fundamental cigenvalue, $w_{1}$. obtains a value of zero, which oceurs when the generalized hardening modulus obtains a value of

$$
H^{a b}=\frac{1}{2} \sqrt{\left(i^{2}+\mu^{2} \beta^{2} h^{2}+h\left(i\left(\mu^{2}+\beta^{2}\right)\right.\right.}-\frac{1}{2}\left(i-\frac{1}{2} \mu \beta h\right.
$$

Any diffuse or discontimuous bifureation mode can be written as a lincar combination of the rigentensors associaterl with $\mathrm{D}$ ". For a discontimuous bifureation to ocent in the $r_{1}-r_{2}$ plane, $i_{13}, i_{23}$ and $i_{33}$ must all equal zero. The conditions $i_{13}=0$ and $i_{23}=0$

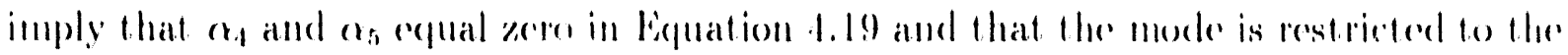
lollowing lorm

$$
\dot{\epsilon}=\mathbf{x}_{1}+a_{2} x_{2}+a_{3} x_{3}+a_{1 ;} x_{1 ;}
$$

(or

$$
\dot{\boldsymbol{\epsilon}}=\left(\gamma_{1}+\alpha_{6 i} / p_{1}\right) \mathbf{e}_{1}+\alpha_{2} \mathbf{e}_{2}+\alpha_{3} \mathbf{e}_{3}+\left(\gamma_{6 i}+\alpha_{6 i} / p_{6}\right) \mathbf{e}_{6}
$$

subject to the following constraint which is needed to satisfy the recpurement that $i_{33}$ (equals zero

$$
-\frac{1}{\sqrt{3}}\left(\gamma_{1}+\alpha_{6 i} \rho_{1}\right)+\frac{1}{\sqrt{2}} \alpha_{2}+\frac{1}{\sqrt{3}}\left(\gamma_{6}+\alpha_{6 i} \rho_{6 i}\right)=0
$$

The general bifureation condition, Equation 4.20, provides the following additional con. straint:

$$
\omega_{1}=-\alpha_{2}^{2} \omega_{2}-\alpha_{3}^{2} \omega_{3}-\alpha_{13}^{2} \omega_{61}
$$

Ising the above equations and maximizing w with resperet to the independent valiables. $r_{2}, a_{3}$ and $a_{6}$, we obtain equations for the fundanental eigenvalue associated with loss of strong ellipticity. Then by iteratively solving the eigenvalue problem and the above equations with monotonically decreasing values for the hardening modulus, we ielentily the critical hardening modulus for the loss of strong ellipticity.

For this material and loading. Rudnicki and Rice (1975) have obtained the following expression for the hadrening modulus associated with a classical discontimous bifureation

$$
I^{d b}=E\left[\frac{(\mu-\beta)^{2}}{18(1-1)}-\frac{(\mu+\beta-\sqrt{3})^{2}}{36}\right]
$$

The critical hardening moduli predicted by the various criteria for minaxial tonsion are plotted as a function of $\mu$ for various values of $\beta$ in Figure 4.3. Figure 4.3a shows critical values of the dimensionless hardening modulus, $(H / E)$, as a function of the internal friction parameter $\mu$ with the dilatancy parameter 8 fixed at -0.3 . Loading is characterized by decreasing values of $(H / E)$. 'Throughout this chapter, a value of (0.3) is used for Poisson's ratio. 'The general bifureation condition is always reached first. for all values of $\mu \neq \beta$. When $\mu=\beta=-0.3$. the general bifurcation and limit point bifurcation criteria coincide as they should for an associated law. Similarly, the strong ellipticity condition is always attained prior to the classical discontimuons bifuration condition unless $\mu=\beta=-(0.3$ where the two discontinuons bifureation reviteria coincide 


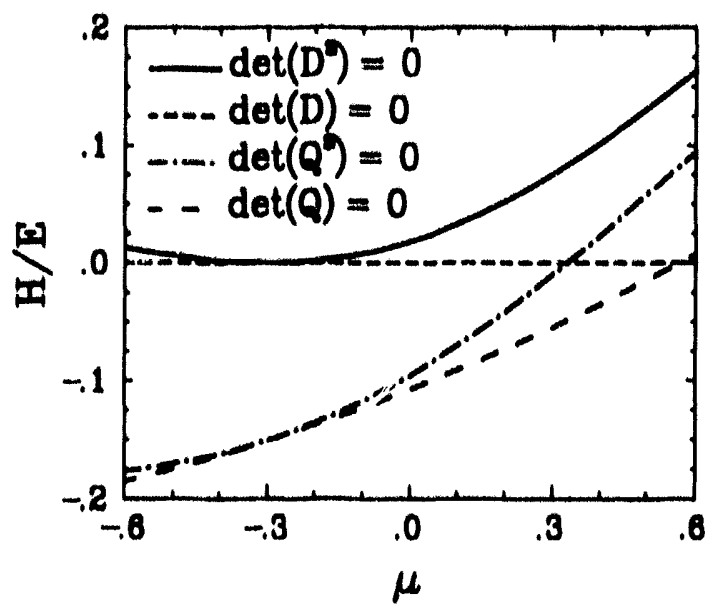

(a) $\beta=-0.3$

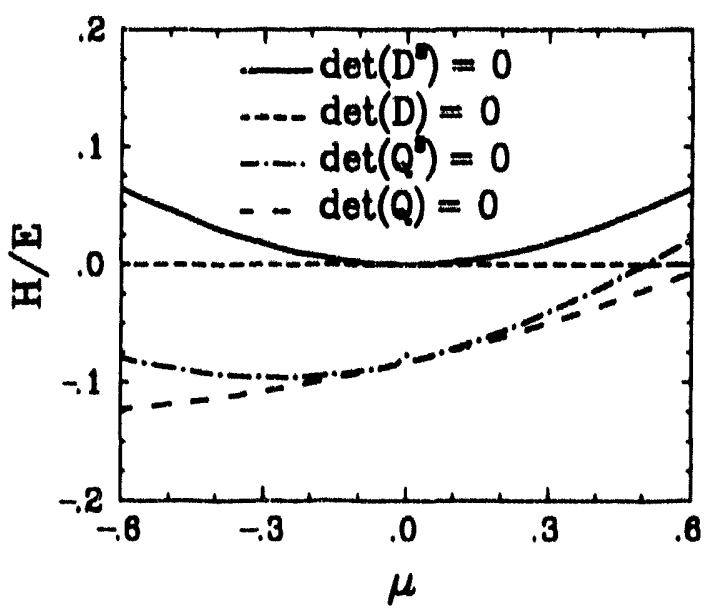

(b) $\beta=0.0$

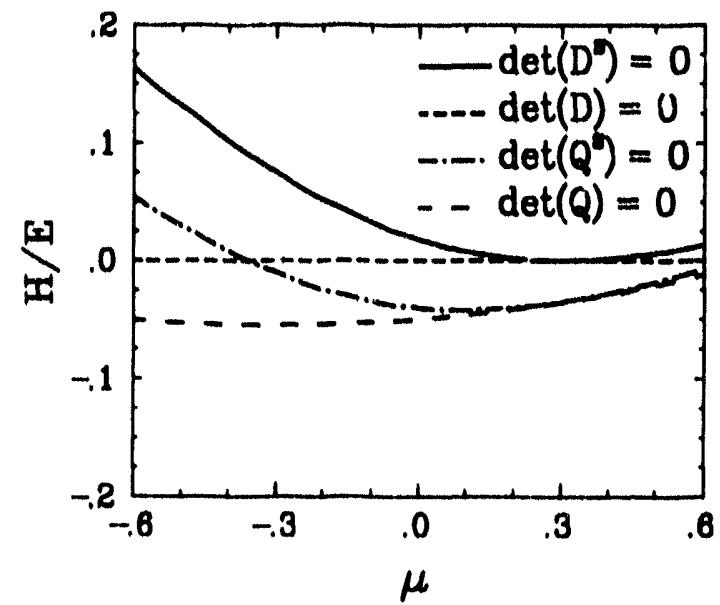

(c) $\beta=0.3$

Figure 4.3. (ritical Hardening Moduli for a Drucker-Prager Material with Non-Assoriated Flow. 
as they should for an assoriated law. Figuress f.3h and f.30 show similar results when the dilatancy parametere of is fixed at 0.0 and 0.3 , resperetively.

All of these results imdicate that the first general bifurcation point is reached in the hardening regime when the flow is non-associative $\mu \neq \|$. When the flow is associalive,

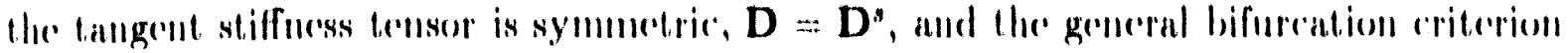
and the limit point bifureation eriterion both identify the limit point as the lirst general bifurcation point. Likewise, when the flow is associative, the classical discontionous bifurcation critcrion and the loss of strong ellipticity criterion identify the sanme point in

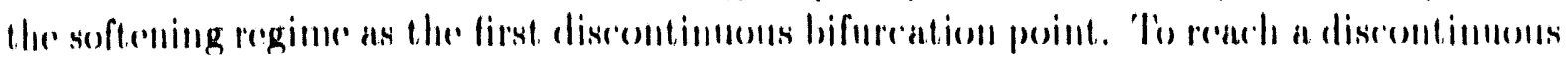
hifuration point, the material must exhibit cither strain-softening or mon-assoriative

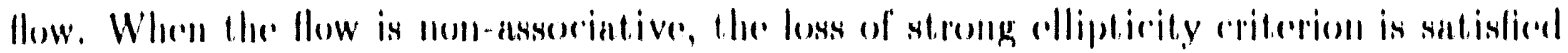

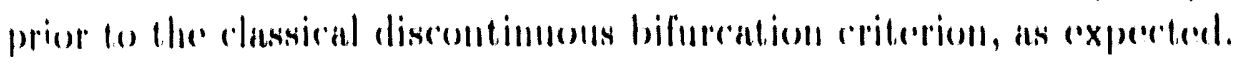

fis allalya a Druckere-Prager malerial with associated flow for a general stress pallh, we call simply repeat the process used in the first part of this section and let $\mu=\beta$.

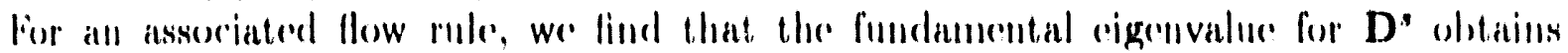
a value of zero when $H$ equals zero and that a megative $/ 1$, strain-softerning, leads tos

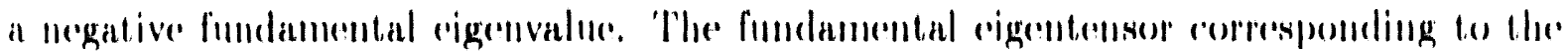
zero rigenvalue at the limit point is

$$
x_{1}=g=\frac{\sigma^{\prime \prime}}{\left(2 \sqrt{T_{2}}\right)}+\frac{\mu}{3} \mathrm{i}
$$

Fome of the rigentensors of $D^{*}$ atre orthogonal to $\mathbf{i}$ and $\boldsymbol{\sigma}^{\prime \prime}$ and span the remaining devi-

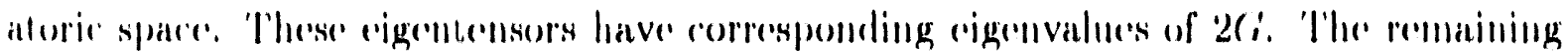
rigentrensor is given by

$$
\mathrm{x}_{1 \mathrm{i}}=\mathrm{i}-\frac{\mu \boldsymbol{\sigma}^{\prime l}}{\sqrt{T_{12}}}
$$

and las a corresponding rigenvalure of

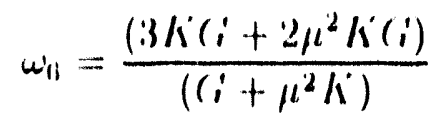

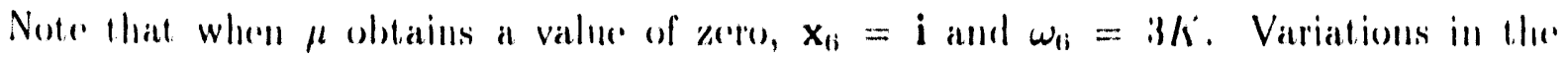

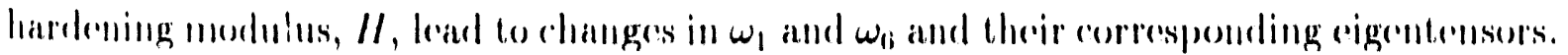
A negative /l leads to a llegative fundamental cigenvalue.

The rritial hardening moduli predicted by the varions criteria for mniaxial tension are plotterl as a function of $\mu$ in Figure 4.4. Note that for an associated flow rule, lhe general hifureation and limit point bifureation criteria both prediet the limit point as the first hifureation point independent of $\mu$. Also, when the flow is associative, the strong ollipticity eriterion and the classieal discontinuons hifureation reviterion generate identical predictions for the amoment of strain-softening needed for localization. For localization to occur, the material must strain-soften or exhibit a significant annomut of pressure dependence. Sperifically, $\mu$ must equal $\sqrt{3} / 2$ for a discontimnous bifurcation to orene without strain-softening. 


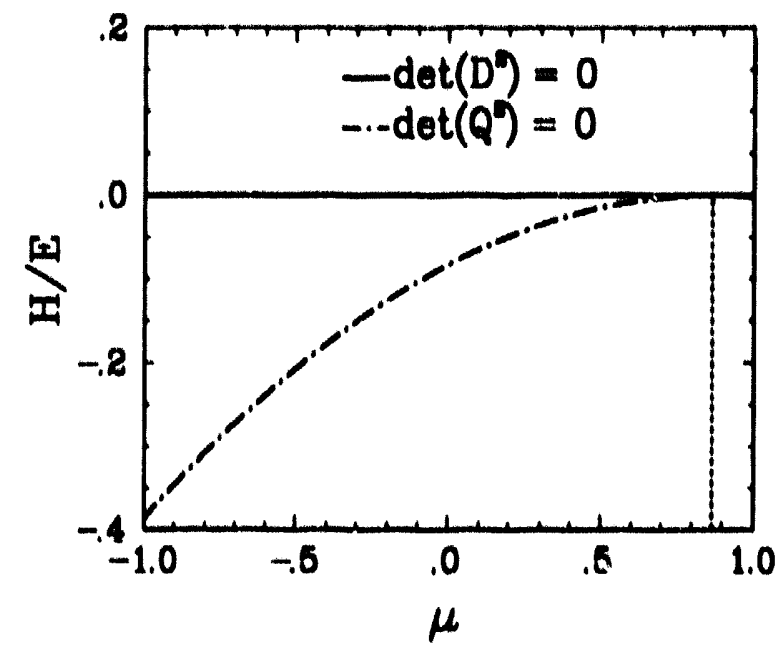

Figure 4.4. ('ritical Inardening, Moduli for a Drucker-Prager Malerial with Assoriated lilow.

\subsection{2 von Mises}

In this sertion, we antalyze a simple voll Mise's plasticity model with associated flow and a yiedd function given by

$$
\Psi=\sqrt{I_{2}}-k
$$

The'll

$$
\mathbf{f}=\mathbf{g}=\frac{\boldsymbol{\sigma}^{\prime}}{\left(2 \sqrt{T_{2}}\right)}
$$

and the tangent stilluess tensor for a plastic step is given by

$$
\mathrm{D}=\mathrm{E}-\frac{2\left(i^{\prime 2}\right.}{(11+(i)} \frac{\sigma^{\prime \prime}(\cdot) \sigma^{\prime \prime}}{\sigma^{\prime \prime}: \sigma^{\prime \prime}}
$$

The plastic tallgent stiffucso tonsor is symunetric, $\mathbf{D}=\mathbf{D}^{*}$.

The fumdanmental eigenterisor for $\mathbf{D}$ is

$$
\mathbf{x}_{1}=\frac{\boldsymbol{\sigma}^{\prime \prime}}{\left(\boldsymbol{\sigma}^{\prime \prime}: \boldsymbol{\sigma}^{\prime \prime}\right)^{\frac{1}{2}}}
$$

with a rorresponding rigenvalue of

$$
\omega_{1}=2\left(i\left(\frac{I I}{H+C_{i}}\right)\right.
$$

which varies from $2(i$ to 0 as $1 /$ varies from $\infty$ to 0 and becomes negative for negative H. 'The remaining eigentensors for $\mathbf{D}$ are a set of four tensors which span the remaining spare of symmetric second-order deviatoric tensors with corresponding eigenvalues of 2 i $^{\prime}$ and the second-order identity with a corresponding eigenvalue of $3 / i$. Note that for this 
model none of the eigentensors and only one rigenvalue, the fumdamental one, deperind ont the hardening modulus, H. The fundamental eigentensor does, however, deperted ont the corrent stress state.

A general bifurcation may first ocenr when the fundamental cigenvalue ohtains a valuse of zero. At this point, the hardening modulus, H, eguals zero. The rharacter of the bifurcation is given by the funclamental eigentensor. For example, when the material is subjerted to miniaxial tension,

$$
x_{1} \Rightarrow \frac{1}{\sqrt{3}}\left[\begin{array}{ccc}
-1 & 0 & 0 \\
0 & 2 & 0 \\
0 & 0 & -1
\end{array}\right]
$$

which represents a general bifureation since $x_{1}$ has no zero eigenvalues. If constraints are present, the material may be loaded into a strain-softening regime withont exhibiting localized deformation associated with the first general bifurcation mode. In the strain-softening regime, the fundamental eigonvalue is negative, and numerons alternate. bifurcation modes may be artivated.

Any bifurcation morle can be written as a linear combination of the eigentensons as given in Eequation 4.19. For a discontinuone bifurcation to ocenr in the $x_{1}-x_{2}$ plane, $i_{13,3}, i_{2: 3}$ and $i_{33}$ must all equal zero. This implies that $\alpha_{1}$ and $\alpha_{5}$ equal zero and the morlo is restricted to the following form:

$$
\dot{\epsilon} \Rightarrow \frac{1}{\sqrt{6}}\left[\begin{array}{ccc}
-1 & 0 & 0 \\
0 & 2 & 0 \\
0 & 0 & -1
\end{array}\right]+\frac{\alpha_{2}}{\sqrt{2}}\left[\begin{array}{ccc}
-1 & 0 & 0 \\
0 & 0 & 0 \\
0 & 0 & 1
\end{array}\right]+\frac{\alpha_{3}}{\sqrt{2}}\left[\begin{array}{lll}
0 & 1 & 0 \\
1 & 0 & 0 \\
0 & 0 & 0
\end{array}\right]+\frac{\alpha_{6}}{\sqrt{3}}\left[\begin{array}{lll}
1 & 0 & 0 \\
0 & 1 & 0 \\
0 & 0 & 1
\end{array}\right]
$$

subject to the constraint.

$$
\frac{-1}{\sqrt{6}}+\frac{\alpha_{2}}{\sqrt{2}}+\frac{\alpha_{63}}{\sqrt{3}}=0
$$

which is an e(puation for a straight line in the $\alpha_{2}-\alpha_{03}$ plane. Note that $\alpha_{3}$ is arbitrary. The general bifurcation condition, Equation 4,20, gives the following additional constraint

$$
2 C \alpha_{2}^{2}+2 C \alpha_{3}^{2}+3 k \alpha_{0}^{2}=-\omega_{1}
$$

which is the equation for an ellipse in the $\alpha_{2}-\alpha_{63}$ plane. Note that when $\omega_{1}$ equals zero, the general bifurcation regime is a single point with $\alpha_{2}=\alpha_{3}=\alpha_{6}=0$. As $H$ and $\omega_{1}$ becone negative, the size of the general bifurcation regime grows. The first discontinnons bifurcalion mode is reached when $H$ obtains a value of $-E / 12$. At this point, $\gamma_{2}=$ $\sqrt{3}(1+v) /(5-v), \alpha_{63}=\sqrt{2}(1-2 v) /(5-v)$, and $\alpha_{33}=0$ (Figure 4.5). ()ttosen and Runesson (1991) analyzed the arcoustic tensor and also showed that the first discontinuons bifurcation mode is reached at this point for a von Mises material subjected to muiaxial tension. If the hardening modulus decreases beyond $-E / 12$ additional discontinnons bifurcation modes may be artivated. 


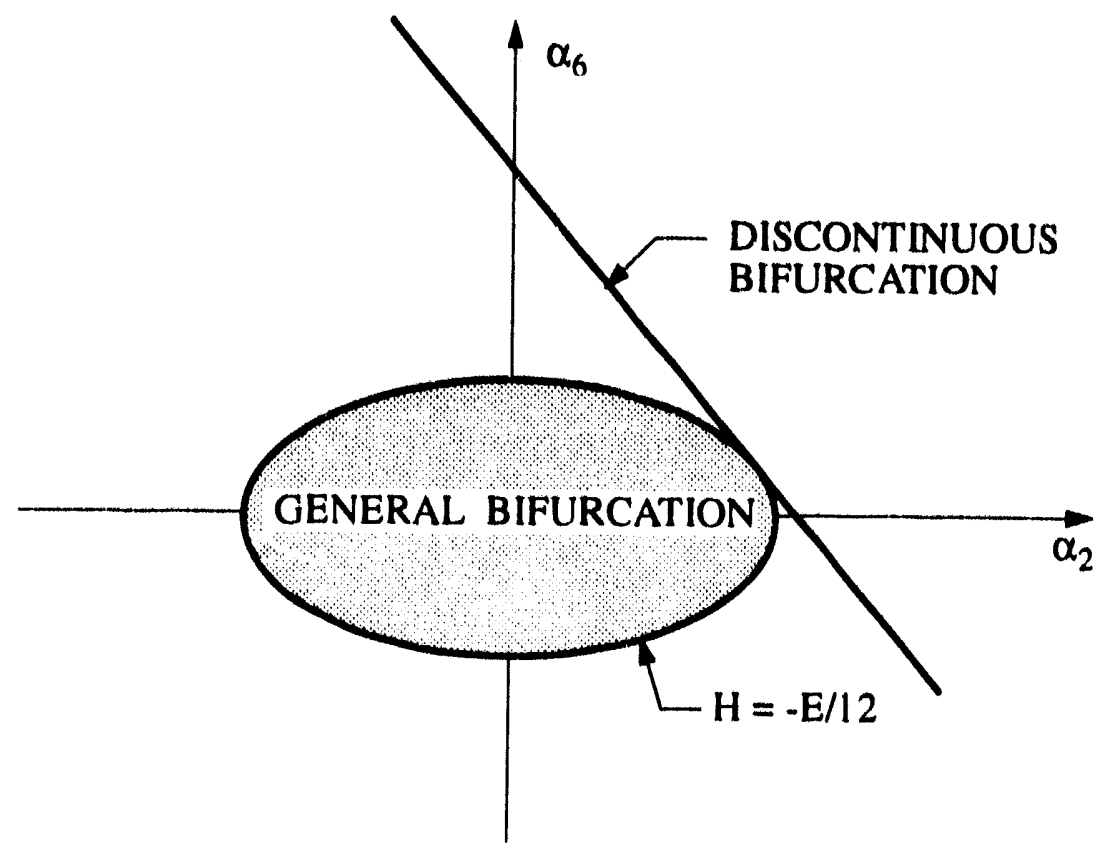

Figure 4.5. Bifurcation Regimes for a von Mises Material Subject to Uniaxial Tension.

Since the fundamental eigentensor depends on the stress state, the amount of softening needed to reach a discontinuous bifurcation will also depend on the stress state. For example, viten the von Mises material is subjected to a pure shear stress in the $x_{1}-x_{2}$ plane, the components of the fundamental eigentensor are

$$
\mathbf{x}_{1} \Rightarrow \frac{1}{\sqrt{2}}\left[\begin{array}{ccc}
-1 & 0 & 0 \\
0 & 1 & 0 \\
0 & 0 & 0
\end{array}\right]
$$

which indicates that the necessary condition for a discontinuous bifurcation is satisfied at the limit point when $H=0$. The important point here is that by altering the stress path, the amount of strain-softening needed to satisfy the necessary conditions for a discontinuous bifurcation can be significantly changed. This occurs because the fundamental eigentensor for this material model depends on the stress state.

\subsubsection{Mohr-Coulomb}

Next, consider a simole Mohr-('oulomb model with a yield function given by

$$
\Psi=\frac{1}{2}\left(\sigma_{1}-\sigma_{2}\right)+\frac{1}{2}\left(\sigma_{1}+\sigma_{2}\right) \sin \phi-\cos \phi
$$

where $\phi$ is the internal friction angle, $c$ is the cohesion, and the coordinate system is chosen such that $\sigma_{1}$ and $\sigma_{2}$ are the maximum and minimum principal stresses, respectively. Then 
for an associated flow law

$$
\mathbf{f}=\mathbf{g} \Rightarrow \frac{1}{2}\left[\begin{array}{ccc}
1+\sin \phi & 0 & 0 \\
0 & -1+\sin \phi & 0 \\
0 & 0 & 0
\end{array}\right]
$$

and the tangent stiffness tensor for a plastic step is again given by Equation 4.26. For this model, the bifurcation at the limit point, $H=0$, is always a discontinuous bifurcation since the fundamental eigentensor for $D^{s}$ is equal to $f$ which has the characteristics required for a discontinuous mode. The nature of the discontinuous bifurcation, as given by the eigentensor, indicates that the components of $\mathbf{n}$ and $\mathbf{m}$ are as follows:

$$
\mathbf{n} \Rightarrow\left\{\begin{array}{c}
\sqrt{1+\sin \phi} \\
\sqrt{1-\sin \phi} \\
0
\end{array}\right\}=c\left\{\begin{array}{c}
1 \\
\tan \left(\frac{\pi}{4}+\frac{\phi}{2}\right) \\
0
\end{array}\right\}, \quad \mathbf{m} \Rightarrow \frac{1}{2}\left\{\begin{array}{c}
\sqrt{1+\sin \phi} \\
-\sqrt{1-\sin \phi} \\
0
\end{array}\right\}
$$

It is not surprising that the Mohr-Coulomb model predicts a discontinuous bifurcation at the limit point and that the orientation of the localized zone depends on the internal friction angle because this model was developed to capture this type of failure. A shear localization is obtained when $\phi=0$ and a discontinuous opening mode is obtained when $\phi=\pi / 2$. This analysis indicates that a Mohr-Coulomb model is appropriate for materials that exhibit localization at the limit point with an orientation that is dependent on an internal friction angle.

A Tresca model with associated flow is identical to a Mohr-Coulomb model with the internal friction angle set equal to zero. Thus, the previous results indicate that a Tresca model is appropriate for materials that display localization at the limit point with an orientation of 45 degrees in the plane of maximum and minimum principal stresses. This model does not predict the generation of necking or diffuse bifurcation modes that are sometimes observed experimentally in metals and is, therefore, not appropriate for metals.

\subsubsection{Principal Stress}

In this section, we consider the principal stress theory of Rankine which uses a yield function given by

$$
\Psi=\frac{1}{2}\left(\boldsymbol{\sigma}: \mathbf{P}^{i}: \boldsymbol{\sigma}\right)^{\frac{1}{2}}-h\left(\boldsymbol{\epsilon}^{p}\right)
$$

where $\mathbf{P}^{i}$ is the principal projection operator. This model has a flow law given by

$$
\dot{\epsilon^{p}}=\dot{\omega} \mathbf{g}=\dot{\omega}(\mathbf{n} \cdot \boldsymbol{\sigma} \cdot \mathbf{n}) \mathbf{n} \otimes \mathbf{n}
$$

For proportional loading, the first possible bifurcation occurs at the limit point and is characterized by the fundamental eigentensor for $\mathbf{D}^{s}$. The fundamental eigentensor for $\mathbf{D}^{s}$ is equal to $\mathbf{g}$ which has the characteristics of a discontinuous opening mode bifurcation. Thus, this model predicts localization at the limit point with the orientation of localization given by the orientation of the maximum principal st. sss. 


\subsection{Evaluation of von Mises Plasticity for Metals}

A von Mises plasticity model with associated flow has been used extensively to describe the plastic deformation of metals. This model does an excellent job of capturing the initiation of plastic deformation in metals. In this section, we use the previous analyses to determine if there is any relationship between the bifurcations predicted by this model and experimentally observed necking and localized deformations in metals. Several different experimental investigations that may enhance an understanding of the necking and localized deformation in metals are reviewed.

\subsubsection{Axisymmetric Rod Subjected to Uniaxial Tension}

First consider a metallic, axisymmetric rod subjected to uniaxial te ssion. The first, type of bifurcation that is generated in metal rods is necking at some section along the length of the rod. In some rods, for example aluminum rods tested at high temperature, (Nadai, 1950), the applied load slowly decreases as the rods continue to neck until the cross-sectional area in the necked region is reduced to a point. In other materials, the initiation of necking is quickly followed by the formation of a crack either at an angle or perpendicular to the applied loading. Here we interpret a crack or the formation of very thin localized deformation zones as evidence of a discontinuous bifurcation, and necking as evidence of a diffuse bifurcation.

Needleman (1972), Hutchinson and Miles (1974) and Miles (1975) have all investigated the necking of rods subjected to uniaxial tension using Hill's (1958) general bifurcation criterion. These researchers have all shown that the initiation of necking is coincident with the attainment of maximum load. The necking in the rod is related to activation of the first general bifurcation mode and is characterized by the fundamental eigentensor, $\mathrm{x}_{1}$, which is equal to the normalized deviatoric stress tensor (Figure 4.6).

Materials that neck until failure do not strain-soften and therefore do not allow for the artivation of any other general or discontinuous bifurcation modes. The fundamental eigentensor associated with the tangent stiffness tensor for a von Mises material is dependent on the stress state. As the necked region evolves, the stress state in the necked region changes. This leads to a local change in the stress deviator and thus a change in the fundamental eigentenso:. Specifically, as necking occurs, a shear stress component is introduced which directly leads to the introduction of a shear strain component in the fundamental eigentensor which explains the formation of a necked region without the generation of any incompatibilities in the neck.

In other materials, a small amount of necking is quickly followed by the formation of a crack either at an angle or perpendicular to the applied loading. The analyses given above indicates that a von Mises material subjected to a pure shear stress can localize without softening but that a von Mises material subjected to a uniaxial tensile stress must exhibit a significant amount of softening to localize. As the necked region evolves 

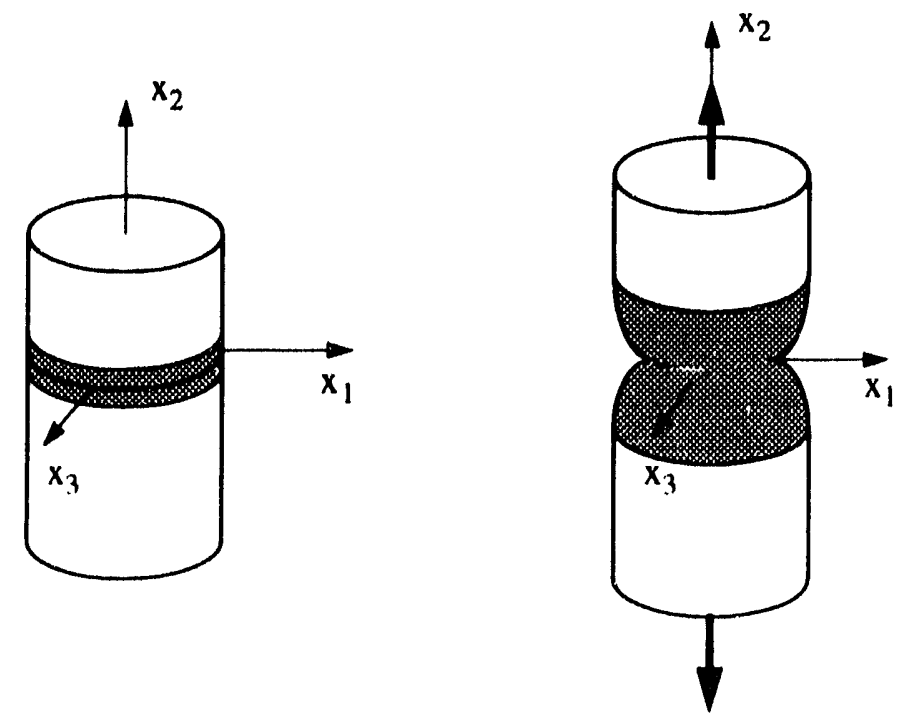

Figure 4.6. Activation of First General Bifurcation Mode in a Rod Subject to Uniaxial Tension.

in the bar, the stress state deviates from a homogeneous uniaxial tensile st,ress stalte and strictly speaking the results from the above analyses do not apply. However, if the: amount of necking is not significant, then it seems reasonable to expect the stress state: in the necked region to approach that of the original homogeneous uniaxial tensile stress state and to conclude that the material must exhibit strain softening for the localization to occur. Of course, the localization could also be due to pressure dependence of the yield surface, non-associated flow, or a combination of softening, pressure dependence, and nonassociativity. An accurate study of the evolution of the necked region and subsequent. potential localization would require a mumerical study similar to that of Necdleman (1972) which would allow a characterization of the stress state in the evolving necked region.

\subsubsection{Axisymmetric Rod with Lateral Displacements Constrained}

Next, consider the same axisymmetric rod subjected to moniaxial tension with the artificial constraint that lateral displacements be identical along the entire length of the bar as shown in Figure 4.7. This constraint, will not allow the bar to neck and will thus constrain the first general bifurcation mode. Furthermore, the first discontimuous bifurcation mode given by Equation 4.57, which is characterized by the formation of a localized zone at an angle of 48.8 degrees from the loading axis will also be constrained. With contimued softening of the material, the first unconstrained discontinuons bifurcation that will actually lead to localization is an opening mode discontinuons bifurcation with an orientation, $\mathrm{n}$, parallel to the loading axis. To activate this mode, $\alpha_{6}$ in Equation 4.57 is equal to $\frac{1}{\sqrt{2}}$, $\gamma_{2}$ and $\alpha_{3}$ are equal to zero, and $H=-E /(6-6 v)$. This example shows that when the first possible discontinuous bifurcation is constrained, the localization that is ultimately generated is not characterized by the mode associated with the first possible discontimusen bifurcation but rather by the first unconstrained discontinuous bifurcation. 

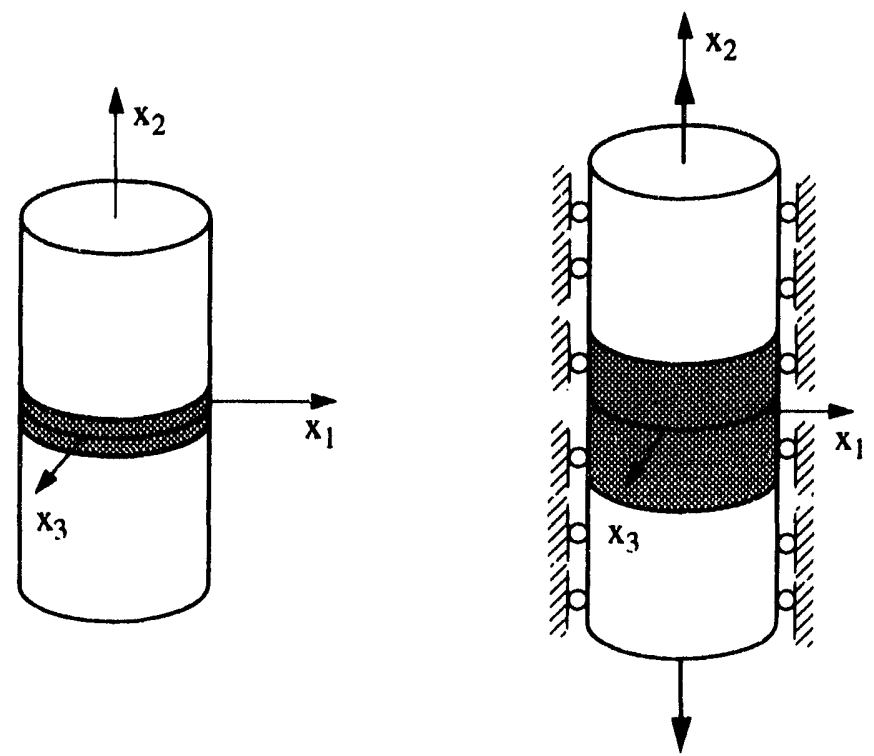

Figure 4.7. Localization in Rod with Lateral Displacements ('onstrained to be Identical.

\subsubsection{Thin Plate Subjected to Uniaxial Tension}

Experiments on thin metal plates subjected to uniaxial in-plane tension indicate that necked regions form at angles of between 55 and 65 degrees from the loading axis (Nadai, 1950; Aronofsky, 1951) and not perpendicular to the applied loading as with axisymmetric. rods. Again, the formation of the neck is sometimes followed by the formation of a crack plane, and at other times the material just continues to neck until failure.

Necking in a thin plate is again associated with the activation of the first general bifurcation and characterized by the fundamental eigentensor. In the rotated coordinate system shown in Figure 4.8, the components of the fundamental eigentensor for $\mathbf{D}^{\text {s }}$ based on Equation 4.54 are

$$
\overline{\mathbf{x}}_{1} \Rightarrow \frac{1}{\sqrt{6}}\left[\begin{array}{ccc}
2 \sin ^{2}(\theta)-\cos ^{2}(\theta) & 3 \cos (\theta) \sin (\theta) & 0 \\
3 \cos (\theta) \sin (\theta) & 2 \cos ^{2}(\theta)-\sin ^{2}(\theta) & 0 \\
0 & 0 & -1
\end{array}\right]
$$

For a thin necked region to form in the plane of the plate, the $x_{1}-x_{2}$ plane, and to remain compatible with the surrounding material, the perturbation to the strain field in the necked region is subject to the constraint that the 11 component of the fundamental eigentensor in the rotated coordinate system must equal zero. This requirement would be that of a discontinuous bifurcation if the third eigenvalue of $\mathbf{x}_{1}$ is zero instead of -1 . Therefore, there is a potential incompatibility in the $x_{3}$ direction. The in-plane constraint yields $2 \sin ^{2}(\theta)-\cos ^{2}(\theta)=0$ or $\theta=35.3$ degrees. In other words, the predicted necked region is oriented at an angle of 54.7 degrees from the loading axis which corresponds to many experimental observations and is identical to the orientation predicted by Nadai (1950) and Thomas (1961) using similar in-plane compatibility arguments. However, these authors did not address the potential incompatibility in the $x_{3}$ direction. The 


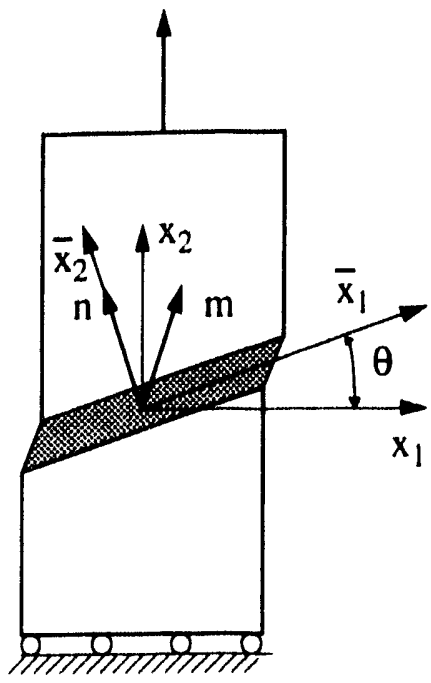

(a) lateral displacement at end

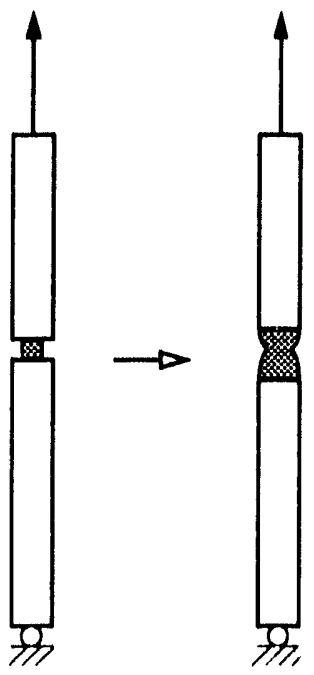

(b) no lateral displacement at end

Figure 4.8. Activation of General Bifurcation Mode in a Plate Subject to Uniaxial Tension.

neck is characterized by the fundamental eigentensor which, for a von Mises material, is dependent on the stress state. As the neck crolves, the stress state in the neck and thus the fundamental eigentensor change. The components of the fundamental eigentensor are expected to vary continuously as a function of location within the neck which explains the formation of the neck without the generation of any incompatibilities which werc introduced and left unexplained in Thomas' (1961) analysis of this thin plate problem.

As shown in Figure 4.8a, the orientation of $\mathbf{m}$ which represents the orientation of relative velorities of regions on opposite sides of the necked region indicates that at least one end of the specimen must be free to move in the $x_{1}$ direction for the neck to occur as shown. Vardoulakis (1978) has developed a device that allows for this lateral displacement; however, many specimens are tested in devices that do not allow lateral displarement. This leads to the formation of two necked regions and relative motion of the bodies on opposite sides of the necks as shown in Figure 4.8b.

Aronofsky (1951) shows that the variation in the orientation of the necked region from 25) to 35 degrees could be due to material anisotropy. The analysis presented in the previous sections allows an alternative explanation of this phenomenon if the loading device provides a constraint which allows the material to be loaded into a strain softening regime. For example, consider the strain perturbation given by

$$
\dot{\boldsymbol{\epsilon}}=\mathbf{x}_{1}+\frac{1}{\sqrt{2}} \beta \mathbf{x}_{6}
$$

which satisfies the instability condition of Equation 4.20. Components of the strain 


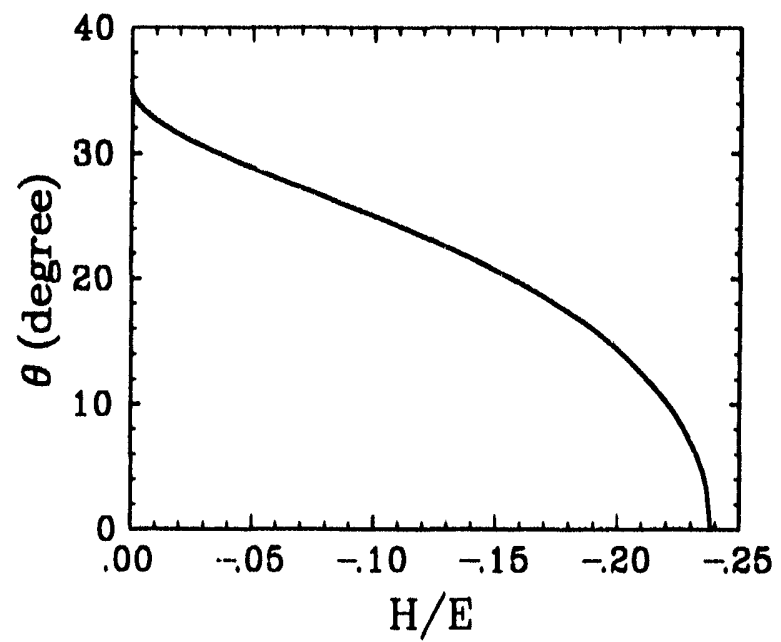

Figure 4.9. Effert of Strain-Softening on the Predicted Orientation of Localization.

perturbation in the rotated coordinate system become

$$
\dot{\boldsymbol{\epsilon}} \Rightarrow \frac{1}{\sqrt{6}}\left[\begin{array}{ccc}
2 \sin ^{2}(\theta)-\cos ^{2}(\theta)+\beta & 3 \cos (\theta) \sin (\theta) & 0 \\
3 \cos (\theta) \sin (\theta) & 2 \cos ^{2}(\theta)-\sin ^{2}(\theta)+\beta & 0 \\
0 & 0 & -1+\beta
\end{array}\right]
$$

The predicted orientation of the necked region is plotted as a function of the amount of softening needed to satisfy the general bifurcation condition in Figure 4.9.

When the hardening modulus, $H$, has a value of $-E / 10$ an orientation of 25 degrees (65) degrees from the loading axis) is predicted. Even less strain soltening is needed to activate modes with orientations between 25 and 35 degrees. These results suggest that the experimentally observed variations in orientation could be caused by the combination of loading constraints and some strain-softening. Also, when the hardening modulus obtains a value of $-E /(6-6 v)$ an orientation of 0 degrees and a discontinuous opening mode bifurcation is predicted which corresponds to localization perpendicular to the applied loading. Such a localization is sometimes observed experimentally which would suggest that some metals must exhibit a significant amount of strain softening, pressure dependence or non-associativity at failure.

\subsubsection{Thin Plate Subjected to Equal Biaxial Tension}

Metal sheets are often formed by subjecting them to equal biaxial tension with a hemispherical punch. At some point in the forming process intersecting shear hands form at angles through the thickness (Beaver, 1983) as shown in Figure 4.10. The necking that is generated prior to localization in thin plates subjected to uniaxial tension is not observed in thin plates subjected to equal biaxial tension. Thus, the change in load path to equal biaxial tension apparently inhibits the activation of any diffuse bifurcation modes prior to localization. 


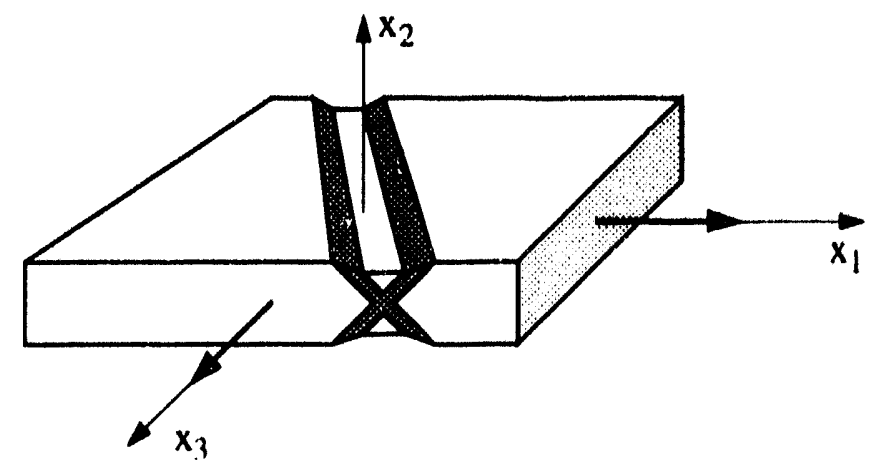

Figure 4.10. Activation of Discontinuons Bifurcation Mode in a Thin Plate Subjert to Eyual Biaxial Tension.

Storen and Rice (1975) suggest that that localization of plates subject to erpal hiaxial tension provides experimental evidence of the formation of vertices in the yield surface. Recently, Hill (1991) has shown that anisotropic hardening condd also lead to the observed localization. Here we present an alternative explanation. For equal hiaxial tension in the $x_{1}-x_{3}$ plane (the plane of the plate), the components of the stress tensor and the stress deviator prior to localization are as follows:

$$
\boldsymbol{\sigma} \Rightarrow \sigma\left[\begin{array}{lll}
1 & 0 & 0 \\
0 & 0 & 0 \\
0 & 0 & 1
\end{array}\right], \quad \boldsymbol{\sigma}^{d} \Rightarrow \frac{\sigma}{3}\left[\begin{array}{ccc}
1 & 0 & 0 \\
0 & -2 & 0 \\
0 & 0 & 1
\end{array}\right]
$$

For this problem the fundamental eigentensor which is the normalized stress deviator is not compatible in the phane of the plate becanse the eigenvalues associated with the $x_{1}-x_{3}$ plane are both positive. Thus, no orientation can be found for which a porturbation to the strain rate field given by the fundamental eigentensor is compatible in the $r_{1}-r_{3}$ plane with the surrounding material. As the material begins to strain soften, mumerons alternate bifurcation modes may be artivated. However, by considering all linear combinations of the eigentensors associated with $\mathbf{D}^{*}$, we quickly find that the first. bifurcation mode which satisties the constraint to remain compatible in the $x_{1}-r_{3}$ plane is a discontinuous bifurcation given by

$$
\dot{\boldsymbol{\epsilon}} \Rightarrow \frac{1}{\sqrt{6}}\left[\begin{array}{ccc}
1 & 0 & 0 \\
0 & -2 & 0 \\
0 & 0 & 1
\end{array}\right]+\frac{\alpha_{2}}{\sqrt{2}}\left[\begin{array}{ccc}
-1 & 0 & 0 \\
0 & 0 & 0 \\
0 & 0 & 1
\end{array}\right]+\frac{\alpha_{6}}{\sqrt{3}}\left[\begin{array}{lll}
1 & 0 & 0 \\
0 & 1 & 0 \\
0 & 0 & 1
\end{array}\right]
$$

which satisfies the instability condition of Equation 4.20. This discontinuous bifurcation mode is activated when $I I=-E / 12, \alpha_{2}=-\sqrt{3}(1+v) /(5-v)$ and $\alpha_{6}=-\sqrt{2}(1-$ $2 v) /(5-v)$. This bifurcation mode represents shear bands forming at angles of 48.8 degrees from the $x_{2}$ axis which is exactly the type of localization that was observed by Beaver (1983). The formation of intersecting shear bands leads to an apparent necking due to the relative motion of material on opposite sides of the shear bands (Figure 4.10). lustead of the requirement of vertices (Storen and Rice, 1975) or of anisotropic hardening 

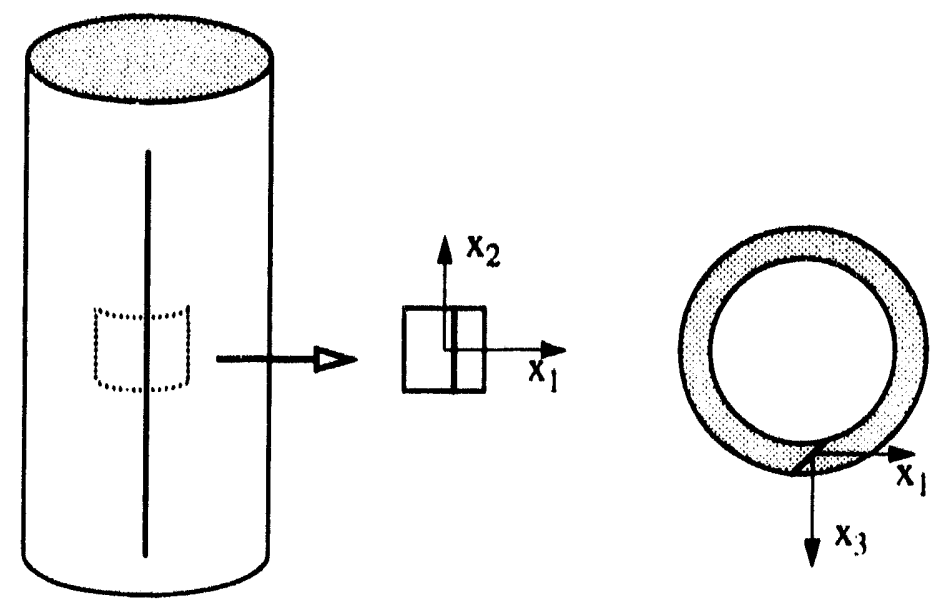

Figure 4.11. Activation of a Discontinuous Bifurcation at the Limit Point in a Pressurized ('ylindrical Pressure Vessel.

(Hill, 1991), this analysis shows that with a sufficient degree of softening, conventional von Mises plasticity with associated flow can predict the localization in a plate subject to equal biaxial tension. Furthermore, this analysis helps to explain why the necking that is observed in thin plates subject to miaxial tension is not observed in thin plates subjected to equal biaxial tension.

\subsubsection{Thin-Walled Cylindrical Pressure Vessel}

Several investigations (e.g., Needleman and Tvergatard, 1984), have involved thin-walled cylindrical pressure vessels subjected to internal pressure. These pressure vessels fail rather catastrophically, with cracks forming along the axis of the cylinder and at an angle through the thickness (discontinuous bifurcation) as shown in Figure 4.11.

Prior to localization the components of the stress tensor and the stress deviator are as follows:

$$
\boldsymbol{\sigma} \Rightarrow \sigma\left[\begin{array}{ccc}
1 & 0 & 0 \\
0 & .5 & 0 \\
0 & 0 & 0
\end{array}\right], \quad \boldsymbol{\sigma}^{d} \Rightarrow \frac{\sigma}{2}\left[\begin{array}{ccc}
1 & 0 & 0 \\
0 & 0 & 0 \\
0 & 0 & -1
\end{array}\right]
$$

We see that the stress deviator is different from the stress deviator found in the previous examples. For this example, the fundamental eigentensor for $\mathbf{D}^{s}$ which is equal to the normalized stress deviator represents a discontinuous bifurcation mode. Furthermore, the fundamental eigentensor indicates that a shear band oriented at 45 degrees throngh the thickness and along the axis of the specimen will occur at the limit point. This predicted localization was observed experimentally by Needleman and Tvergaard (1984). (omparing this result with the previous ones, we see that the stress state generated in the wall of a cylindrical pressure vessel has a detrimental effect on the apparent ductility of the material. 
These exampless show that the type of hifureation, diffuse or discontimusus, exhibiterl by metals apparently depends not only on the material but also on the geomentry and the pre-bifurration stress state. $\Lambda$ simple von Mises plasticity model with assodiated flow predicts both the necking and the localization that is observed experimentally. 'This allalysis indicates that strain softening cant accoment for many foatures observerd in metals. Most previons analyses have focused primarily on pressure dependence, nomassociativity

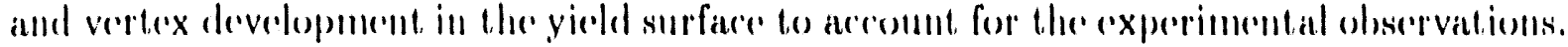

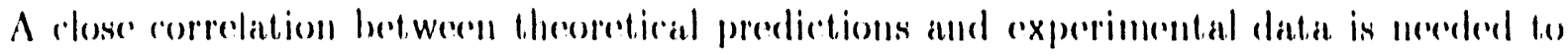
provide answers concerning which effect is dominant lor any given material.

\subsection{Bifurcation Analysis of Continuum Damage Models}

In this sertion, bifureation andyses are performed on varions rontimmom damage theories

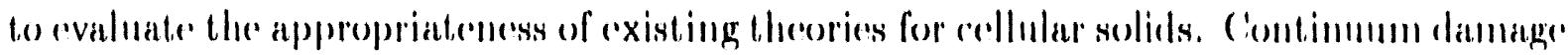
heories have a constitutive relation of the form

$$
\boldsymbol{\sigma}=\mathbf{S}: \boldsymbol{\epsilon}
$$

where $\boldsymbol{\sigma}$ is the total stress temsor, $\mathbf{S}$ is the damaged constic stiffuess tensor and $\boldsymbol{E}$ is the total strain tensor. 'To determine if additional damage is being gencrated during the current load step, we postulate the existence of a damage function, $\Psi$, which defines a surfare in stress or strain spare separating the elastic and damaging proressess

$$
\Psi=\frac{1}{2} \boldsymbol{\sigma}: \mathbf{P}: \boldsymbol{\sigma}-g(\mathbf{S}) \quad \Psi=\frac{1}{2} \boldsymbol{\epsilon}: \mathbf{R}: \boldsymbol{\epsilon}-\eta(\mathbf{S})
$$

where $\mathbf{P}$ is a positive-definite fondth-order tensol that defines the model and $\mathbf{R}=\mathbf{S}: \mathbf{P}$ : S. We also postulate the existence of an evolution equation for $\mathbf{S}$ which is analogons to a llow rule in plasticity. $A$ possible damage evolution equation is given by

$$
\dot{\mathbf{S}}=-\dot{\omega} \mathbf{R}
$$

where $\mathbf{R}$ is a fomrth-order tensor which defines the orientation of the dannage increment. and $\omega$ a monotonically increasing parameter that characterizes the magnitode of the damage increment. Note that hoth the damage function and the evolution refuation have beren rhosent to nse the fomrth-order ternsor $\mathbf{R}$.

Ising the consistency condition, the tangent stiffness tensor, D, for a damaging proress such that $\dot{\sigma}=\mathrm{D}: \dot{\boldsymbol{\epsilon}}$ is obtained

$$
\mathbf{D}=\mathbf{S}-\frac{1}{A}(\mathbf{R}: \boldsymbol{\epsilon} \cos : \mathbf{S})
$$

where $f$ is the normal to the damage surface defined by the damage function $\Psi$ :

$$
\mathbf{f}=\frac{\partial \Psi}{\partial \boldsymbol{\sigma}}=\mathbf{P}: \boldsymbol{\sigma}
$$




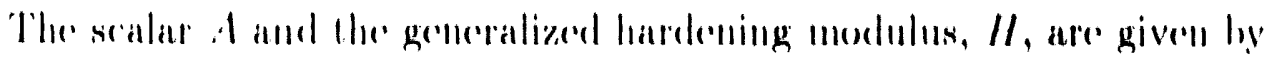

$$
\therefore=H+\mathbf{f}: \mathbf{R}: \boldsymbol{\epsilon} \quad \|=\frac{\partial \Psi}{\partial \mathbf{S}}:: \mathbf{R}
$$

The hardening modulus, $I I$, is positive, zero or negative for strain hardening, perferet, and

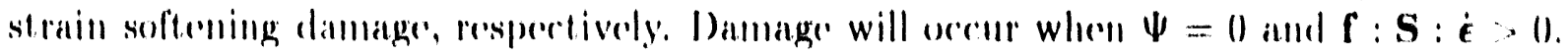

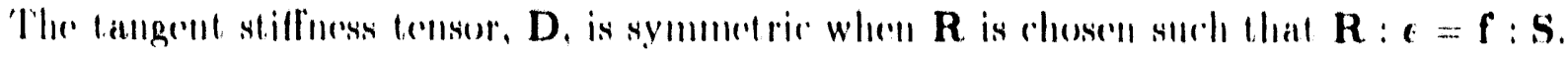

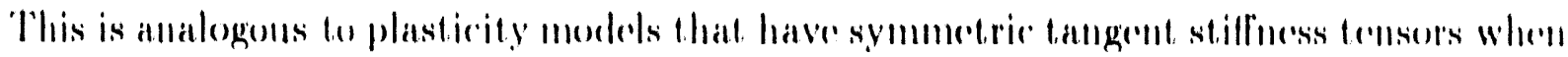

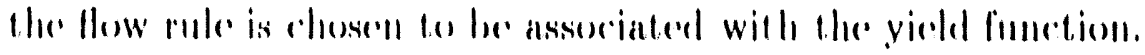

\subsubsection{Simple Scalar Damage}

In this section, we analyze a simple isotropic, scalar damage model with the following damage function:

$$
\Psi=\frac{1}{2} \epsilon: \mathbf{E}: \epsilon-!(\mathbf{S})
$$

and an evolution edpation for the clastic stilfuess ternsor as follows:

$$
\dot{\mathbf{S}}=-\dot{\omega} \mathbf{E}
$$

The tangent stiffuess tomsor for a damaging process is given by

$$
\mathbf{D}=\mathbf{S}-\frac{1}{2 \Pi(1-w)^{2}+\epsilon: \boldsymbol{\sigma}}(\boldsymbol{\sigma} \cos )
$$

Since the tangent stilluess tonsor is symmetrie, hifurrations may lirst ocent at the limit point when the hardening modulus, $/ 1$, and the fundamental rigenvalue oblanin a value

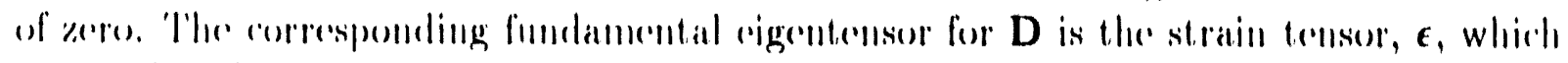
means that the type of hifureation that may be artivated at the limit poimt depends on the strain state. For example, a scalar damaging material subject to a plane strain romdition womld exhihit a discontimuses bifureation and localization at the limit point, hut the

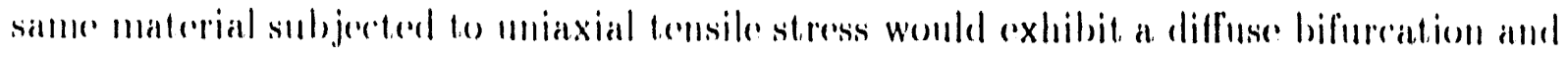
merking at the limit point.

\subsection{2 von Mises Damage}

In this serdion, we analyze a simplo von Mises damage model which has a damage function that is identical to the yiold function for von Mises plasticity

$$
\Psi=\frac{1}{2} \boldsymbol{\sigma}: \mathbf{P}^{d l}: \boldsymbol{\sigma}-g(\mathbf{S})
$$

The damage evolution equation is chatacterized by

$$
\mathbf{f}=\mathbf{P}^{\prime \prime}: \boldsymbol{\sigma}=\boldsymbol{\sigma}^{\prime \prime} \quad \mathbf{R}=4 c^{\prime 2} \mathbf{P}^{\prime l}
$$


and the tangent stifluess tertesor is given by

$$
\mathbf{D}=\mathbf{S}-\frac{4\left(i^{2}\right.}{\left(2\left(i \boldsymbol{\sigma}^{\prime \prime}: \boldsymbol{\sigma}^{\prime}+I I\right)\right.}\left(\boldsymbol{\sigma}^{\prime \prime}(\cdot) \boldsymbol{\sigma}^{d}\right)
$$

The tangent stiffuess tensor is symmetric and has a fundamental cigenvalue and ronresponding cigentensore given by

$$
\omega_{1}=\frac{2(i H}{\left(H+2\left(i \sigma^{d}: \sigma^{d}\right)\right.} \quad x_{1}=\sigma^{\prime \prime}
$$

For this model the first pessible bifureation is characterized by the stress devialeor. 'The type of hifureation that may orcor at the limit point in this material will depenel on the stress state. If the material is subjected to a pure shear stress then localization may ocent at the limit point but if the material is subjected to moxiaxial tension then a diffuse bifurcation leading to nerking may be activated at the limit point. If the material strain softens with contimued loading then mumerous other hifurcation mosles which are written as linear comblomations of the eigentensors as in Equation 4.19 and which satisfy the general bifureation criterion of Equation 4.20 may be artivated.

\subsection{3 von Mises Damage with Alternate Damage Evolution}

In this section, ronsider a von Mises damage model which has a dannge function which is irlentical to the yield function for von Misess plasticity

$$
\Psi=\frac{1}{2} \sigma: \mathbf{P}^{\prime \prime}: \sigma-\eta(\mathbf{S})
$$

alid

$$
f=P^{\prime \prime}: \sigma=\sigma^{\prime \prime}
$$

If we cheose the orientation of the damage incerement to be given hy

$$
\mathbf{R}=\mathbf{S}
$$

then the tangent stiffuess tronsor is as follows:

$$
\mathbf{D}=\mathbf{S}-\frac{2(i}{\left(\boldsymbol{\sigma}^{\prime \prime}: \boldsymbol{\sigma}^{\prime \prime}+I I\right)}\left(\boldsymbol{\sigma}(\cdot) \boldsymbol{\sigma}^{\prime \prime}\right)
$$

The tangent stiffuess tensor for this model is not symmetric. An rigenanalysis of the symmetrie part of this tangent, stiffuess ternsor is more differult than the aigenandysis presenter in the previous section. For this model, the necessary condition for a bifureation is first satisfied in the harrlening regime when 11 obtains a value of

$$
H=\frac{1}{2} \sqrt{\left(\sigma^{\prime l}: \sigma^{\prime \prime}\right)^{2}+\frac{2(i}{3 K^{\prime}}\left(\sigma^{\prime l}: \sigma^{\prime l}\right)\left(\sigma^{s \prime \prime}: \sigma^{s \prime}\right)}-\frac{1}{2}\left(\sigma^{\prime \prime}: \sigma^{\prime \prime}\right)
$$

With this model, bifurrations may ocene in the hardening regime, $I I>0$, muless the stress state is purely deviatoric. When the stress state is purely deviatorice bifurrations may first orene at the limit point. 


\subsubsection{Principal Stress Damage}

In this sertion, we analyze a damage model which uses a principal stress projection operator and a damage evolution ceguation in which the compliance of the material is increased in the direction of maximum principal stress. Consider a model with the damage function

$$
\Psi^{i}=\frac{1}{2} \boldsymbol{\sigma}: \mathbf{P}^{i}: \boldsymbol{\sigma}-g(\mathbf{S})
$$

where $\mathbf{P}^{\prime}$ is the principal projection operator given by

$$
\mathbf{P}^{\prime}=\mathbf{n}^{\prime} \leftrightarrow \mathbf{n}^{\prime}\left(\cdot \mathbf{n}^{\prime} \leftrightarrow \mathbf{n}^{i} \quad .\right.
$$

and $\mathrm{n}^{\prime}$ is a vertor oriented in a principal stress direction. This yield function repressents a rabe in principal stress spare. For proportional loading, the normal to one fare of the dannage surfare is given by $\mathbf{f}^{\prime}$ and the flow rule is characterized by $\mathbf{R}^{\prime}$ where

$$
\mathbf{f}^{i}=\mathbf{P}^{i}: \boldsymbol{\sigma}=\left(\mathbf{n}^{i} \cdot \boldsymbol{\sigma} \cdot \mathbf{n}^{i}\right) \mathbf{n}^{i} \cdot \mathbf{n}^{i} \quad \mathbf{R}^{i}=\mathbf{S}: \mathbf{P}^{i}: \mathbf{S}
$$

'The tangent stiffuess terlnsor is as follows:

$$
\mathbf{D}=\mathbf{S}-\frac{1}{\left(\boldsymbol{\sigma}: \mathbf{P}^{\mathbf{i}}: \mathbf{S}: \mathbf{P}^{\mathbf{i}}: \boldsymbol{\sigma}+H\right)}\left(\mathbf{S}: \mathbf{P}^{i}: \boldsymbol{\sigma}\left(\cdot \boldsymbol{\sigma}: \mathbf{P}^{i}: \mathbf{S}\right)\right.
$$

The tangent stiffuess ternsor for this model is symmetric and at the limit point has a funclanemental eigenvalue of zero with a corresponding fundamental cigentensor given by

$$
\mathbf{x}_{1}=\mathbf{P}^{i}: \boldsymbol{\sigma}=\left(\mathbf{n}^{i} \cdot \boldsymbol{\sigma} \cdot \mathbf{n}^{i}\right) \mathbf{n}^{i}(\cdot) \mathbf{n}^{i}
$$

This rigentensor, $\mathbf{x}_{1}$, has the kinematically compatible form of $\dot{\boldsymbol{\epsilon}}^{k}$ in Equation 4.4. Thus, this morlel predicts localization at the limit point in the form of a discontinnons opening mode bifurcation with an orientation in the direction of the principal stress.

\subsubsection{Principal Strain Damage}

In this section, we antyan a principal strain damage model with a dannge function given by

$$
\Psi^{i}=\boldsymbol{\epsilon}: \mathbf{P}^{i}: \epsilon-h(\mathbf{S}) \quad \Psi^{i}=\sigma: \mathbf{C}: \mathbf{P}^{i}: \mathbf{C}: \boldsymbol{\sigma}-y(\mathbf{S})
$$

where $\mathbf{C}$ is the compliane of the material, $\mathbf{C}=\mathbf{S}^{-1}$, and $\mathbf{P}^{i}$ is the principal projection operator. This yodel function represents a cube in principal strain spare. For proportional loading, the normal to one face of the damage surface is given by

$$
\mathbf{f}=\mathbf{C}: \mathbf{P}^{i}: \mathbf{C}: \boldsymbol{\sigma}
$$

By selerting a damage evolution equation with the following form

$$
\mathbf{R}=\mathbf{P}^{\mathbf{i}}
$$


we obtain a symmertric tangent stilfuess tensor:

$$
\mathbf{D}=\mathbf{S}-\frac{1}{\left(\boldsymbol{\epsilon}: \mathbf{P}^{i}: \mathbf{C}: \mathbf{P}^{i}: \boldsymbol{\epsilon}+H\right)}\left(\mathbf{P}^{i}: \boldsymbol{\epsilon} \cdot \boldsymbol{\epsilon}: \mathbf{P}^{i}\right)
$$

At the limit point, $H=0$, this tangent stiffuess tensor las a fundamental rigenvalue e(pual to zero with a corresponding fundamental eigentensor given by

$$
\mathbf{x}_{1}=\mathbf{C}: \mathbf{P}^{i}: \boldsymbol{\epsilon}
$$

An interesting feature of this model is that the character of the bifurcation depends on the elastic response of the material as reflected throngh $\mathbf{C}$ in Equation 4.99.

The bifurcation analyses presented in this chapter provide a commertion between localization and constitutive theorics. A bifurcation analysis along with careful observation of loralization in material specimens subjert to various loading conditions can provide valuable confirmation of the approprialeness of the constitutive theory chessen for a specifir: material. 


\section{NUMERICAL SIMULATION OF LOCALIZATION}

A large number of researehers have nsed finde element methosls to investigate localization phenomena (Bazant and Lin, 1988; Belytschko, Fish and Engelmann, 1988; delkorst, 1986; Larsson ai: 1 Rumesson, 1991; Leroy and ()rtiz, 1989; Needleman, 1972; Nerelleman and Toergaard, 1984; and (Ortiz, Leroy and Needleman, 1987). These researchers show that diffuse bifureations can he captured using standard finite element coles, but that mesh dependent solutions are ohtained when localization ocenrs. The first seretion in this chapter includes finite clement analyses of bifurceition problems using standard finite clement codes. Following hloese analyses, modifications which enhance ond ability to momerically simmlate localization phenomena are discussed.

\subsection{Analyses with Existing Finite Element Codes}

In this sertion, several quasi-static, boundary value problems are numerically analyzed to determine if existing finite element codes can predict the general and discontinuons bifurcations which are predicted by the bifurcation analyses in the previons section. Several different combinations of geometry, loading and material are considered.

\subsubsection{Rod Subjected to Uniaxial Tension - von Mises Plasticity}

The first series of andyses was performed using three different two-dimensional, axisymmetric models of a rod (Fignere 5.1). The rods were given an initial imperfection by reducing the radius of the finite element mesh one percent near the center. The analy'ses were performed using SANTOSS (Stone, 1992) which is a finite element code that uses dynamir relaxation to solve non-linear, quasi-static problems. A von Mises, olastice perfertly plastic material with the properties given in Table 5.1 were used in these allalysers.

Three different analyses were performed using various element sizes to determine if the solution converged with mesh refinement. Plots of the deformed mesh subject to an axial displacement of 0.05 inches are shown in Figure 5.2. These plots show that the

Table 5.1. von Mises Material Parameters.

$$
\begin{aligned}
& \text { Yomng's Modulus }=30.0 \mathrm{E}+06 \mathrm{psi} \\
& \text { Poisson's Ratio }=0.30 \\
& \text { Yield Strength }=30.0 \mathrm{E}+0.3 \mathrm{psi}
\end{aligned}
$$




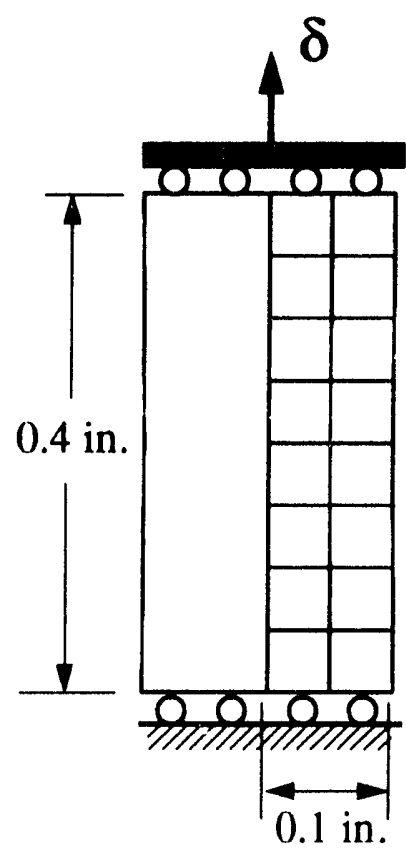

(a) 16 elements

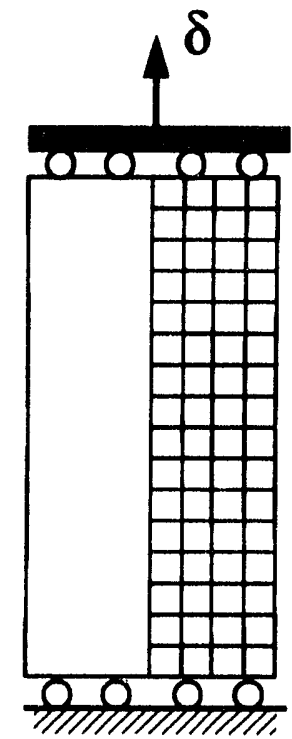

(b) 64 elements

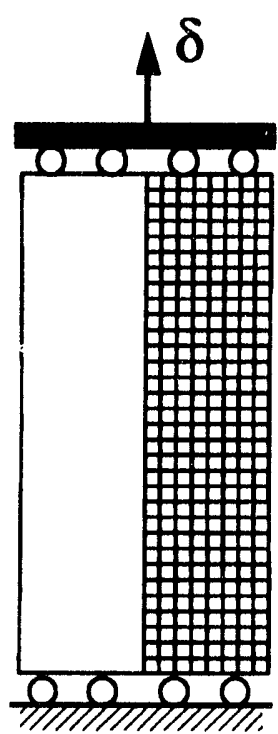

(c) 256 ele:nents

Figure 5.1. Axisymmetric Finite Element Models of a Rod.

necking associated with activation of the first general bifurcation mode is predicted by the finite element analyses. The bifurcation analyses presented in Section 4.7 also predicted activation of the first general bifurcation mode for this problem. Plots of applied load versus end displacement are shown in Figure 5.3. These plots indicate that the numerical results have converged to an accurate solution with mesh refinement.

Next, the effects of strain-softening on the numerical simulation were investigated by analyzing the behavior of rods constructed from strain-softening materials. Two analyses with different amounts of strain-softening were performed using the 256 element model shown in Figure 5.1. In the first softening analysis, the material was given a softening modulus of $-E / 12$ which according to the bifurcation analysis is sufficient to initiate localization at the limit point. A plot of applied load versus end displacement and a plot of the deformed mesh at the last solution step are shown in Figure 5.4. These plots indicate that the solution algorithm remains stable well into the inelastic regime. The contour plots of equivalent plastic strain indicate that localized shear bands may be starting to form at the last stable solution step. The first possible bifurcation is not activated at the limit point in this analysis because it is associated with a mode which is not axisymmetric and this axisymmetric analysis will not allow deformations which are not axisymmetric. If the rod had been represented by a three dimensional model, this bifurcation mode may have been predicted. When the rod was given a softening modulus equal to $-E /(6-6 v)$ the solution algorithm became unstable during the first load step 


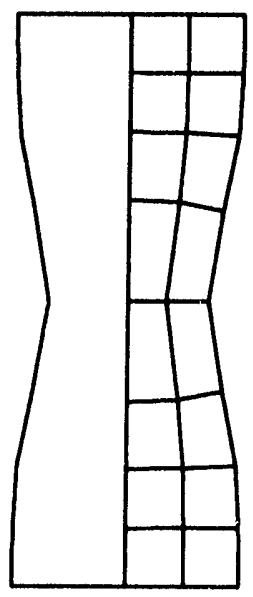

(a) 16 elements

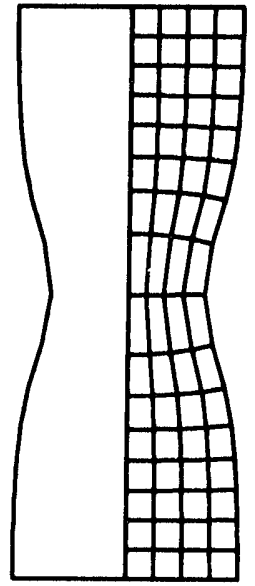

(b) 64 elements

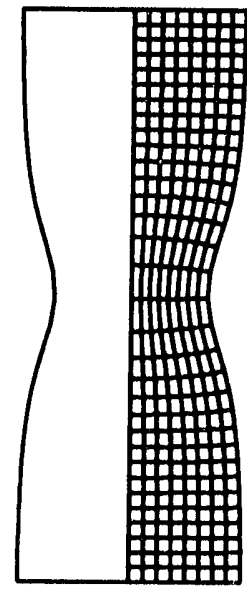

(c) 256 elements

Figure 5.2. Deformed Shape of Finite Flement Models Subject to an Axial Displacement of $0.10 \mathrm{in}$. - Elastic, Perfectly Plastic von Mises Material.

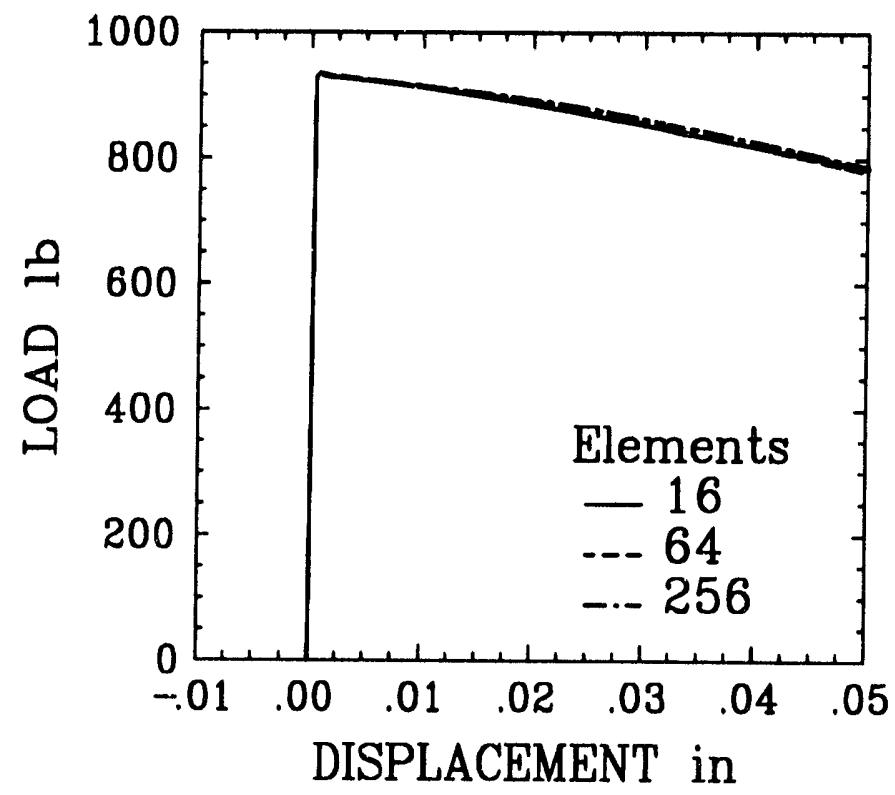

Figure 5.3. Applied Load vs. End Displacement - Elastic, Perfectly Plastic von Mises Material. 
into the softening regime (Figure 5.5.) The contour plot of equivalent plastic strain or the deformed mesh plot at the last solution step provide little information about the reason for the instability. However, the bifurcation analysis presented in the previous section indicates that the initiation of a discontinuous opening mode bifurcation which is axisymmetric should be expected at the limit point in a rod with this amount of softening.

\subsubsection{Rod Subjected to Uniaxial Tension - Principal Stress Plasticity}

The next series of analyses was performed using the same models as in the previous analyses (Figure 5.1); however, in these analyses, an elastic strain-softening material which uses the principal stress criterion of Rankine was used. Material parameters given in Table 5.2 which are appropriate for a brittle foam were used.

Table 5.2. Principal Stress Material Parameters.

$$
\begin{aligned}
& \text { Young's Modulus }=3000 \mathrm{psi} \\
& \text { Poisson's Ratio }=0.0 \\
& \text { Tensile Strength }=300 \mathrm{psi} \\
& \text { Softening Modulus }=-100 \mathrm{psi}
\end{aligned}
$$

Plots of the deformed mesh are shown in Figure 5.6. The 16 and 64 element models have been subjected to an end displacement of 0.06 inches and the 256 element model has been subjected to an end displacement of 0.042 inches. At this point in the simulation, the axial stress in the 256 element model has been nearly reduced to zero due to the large amount of softening in the middle row of elements. These plots show the formation of a discontinuous opening-mode bifurcation in one row of elements. The width of the localized zone depends on the size of the elements being used. The bifurcation analysis of this problem also predicts the initiation of a discontinuous opening mode bifurcation at the limit point. Plots of applied load versus end displacement are shown in Figure 5.7. These plots show that the solution is not converging with mesh refinement and that the predicted applied load versus end displacement depends on the size of the elements used in the simulation.

\subsubsection{Thin Plate Subjected to Uniaxial Tension - von Mises Plasticity}

The next series of analyses were performed using JAC-3D (Biffle, 1389). JAC-3D is a three-dimensional finite element code which uses the conjugate gradient method to solve large deformation, quasi-static, solid mechanics problems. In these analyses, threedimensional models of 3.0 inch square plates which are 0.30 inches thick (Figure 5.8) were subjected to uniaxial tension and equal biaxial tension to determine if the behavior predicted by the bifurcation analyses would be exhibited. The plates were given an initial imperfection by reducing the plate thickness near the center to 0.29 inches.

In the first analysis in this series, a von Mises plasticity theory with the properties given in Table 5.1 was used. The thin plate was subjected to uniaxial tension in the 

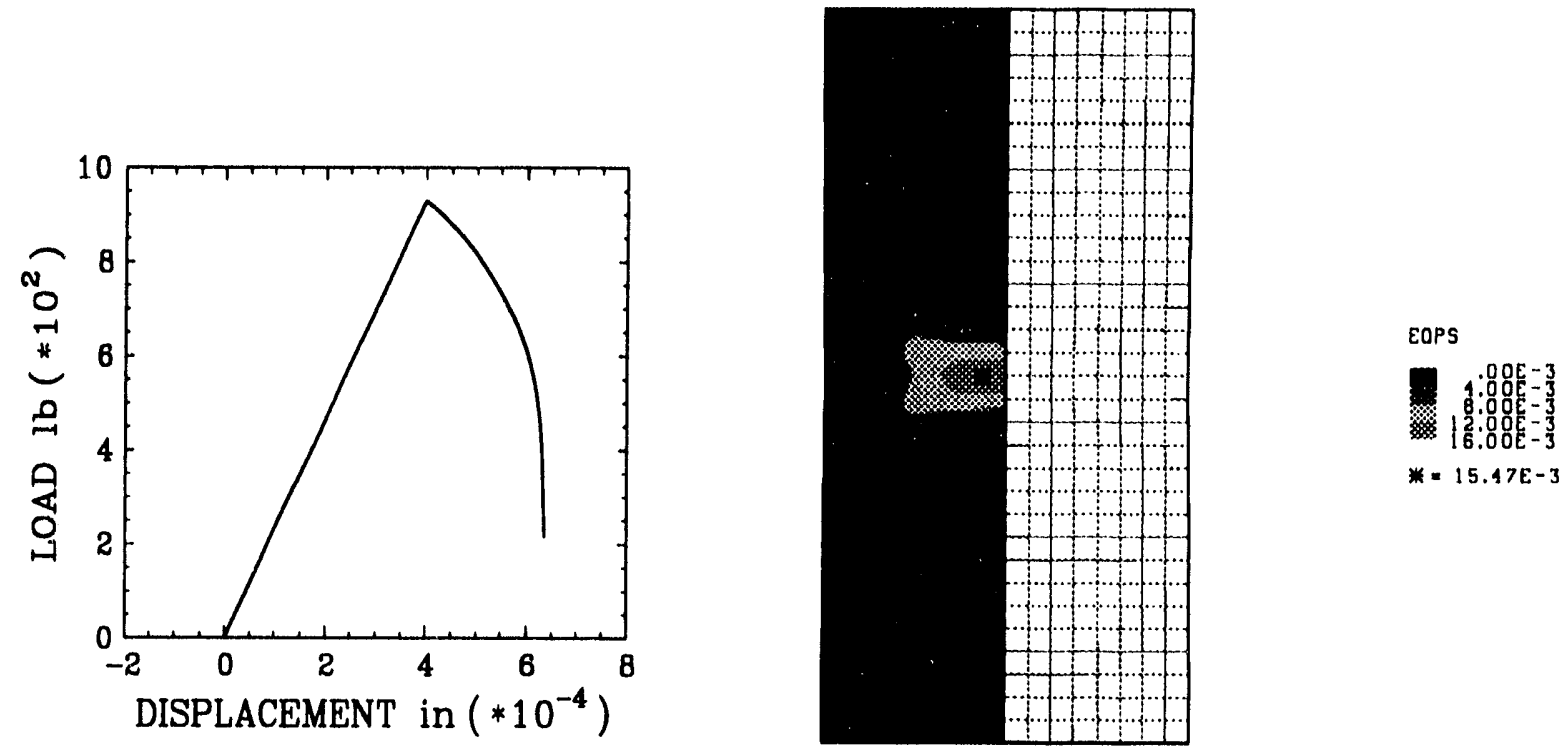

Figure 5.4. Results from Finite Element, Analysis with $\mathrm{H}=-\mathrm{E} / 12$.
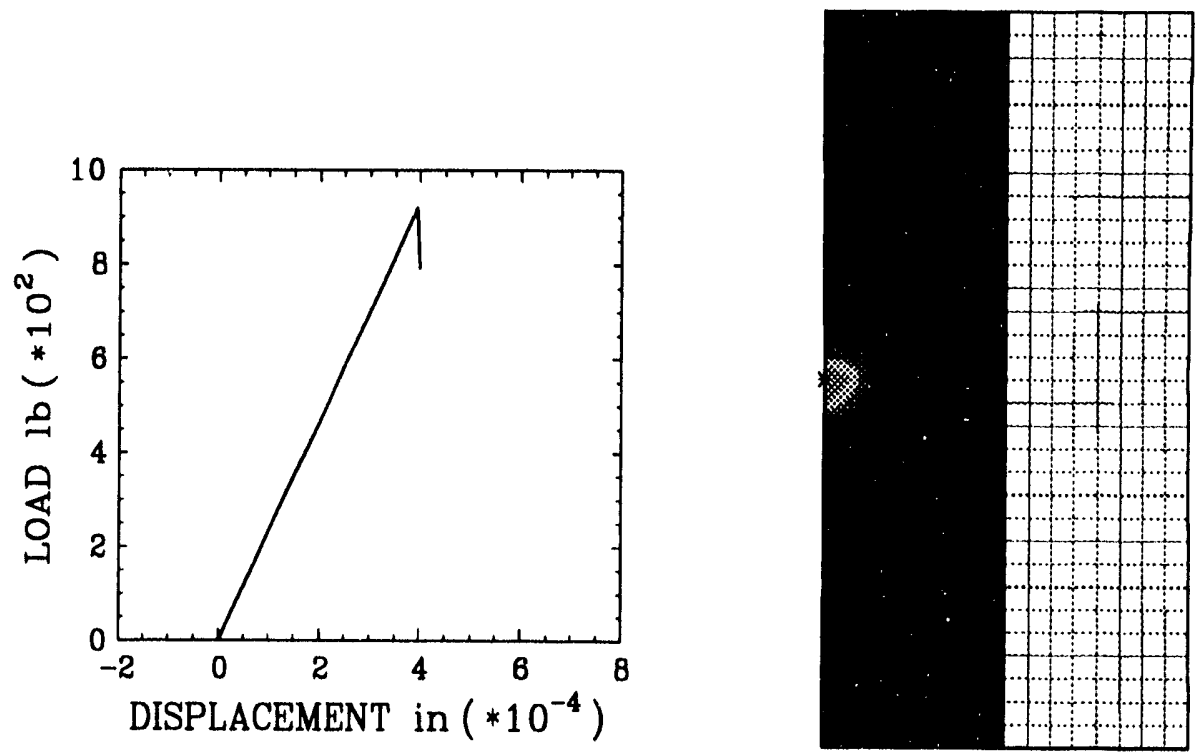

EOPS

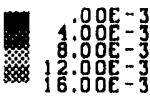

* $=18,3 \mathrm{BE}-$

Figure 5.5. Results from Finite Element Analysis with $\mathrm{H}=-\mathrm{E} /(6-6 \mathrm{v})$. 


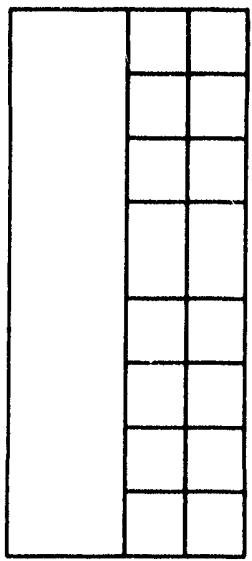

(a) 16 elements

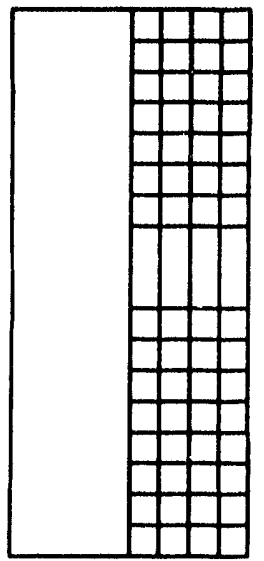

(b) 64 elements

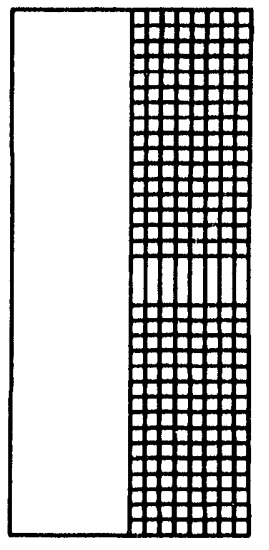

(c) 256 elements

Figure 5.6. Deformed Shape of Finite Element Models Subject to Uniaxial Tension Principal Stress Plasticity.

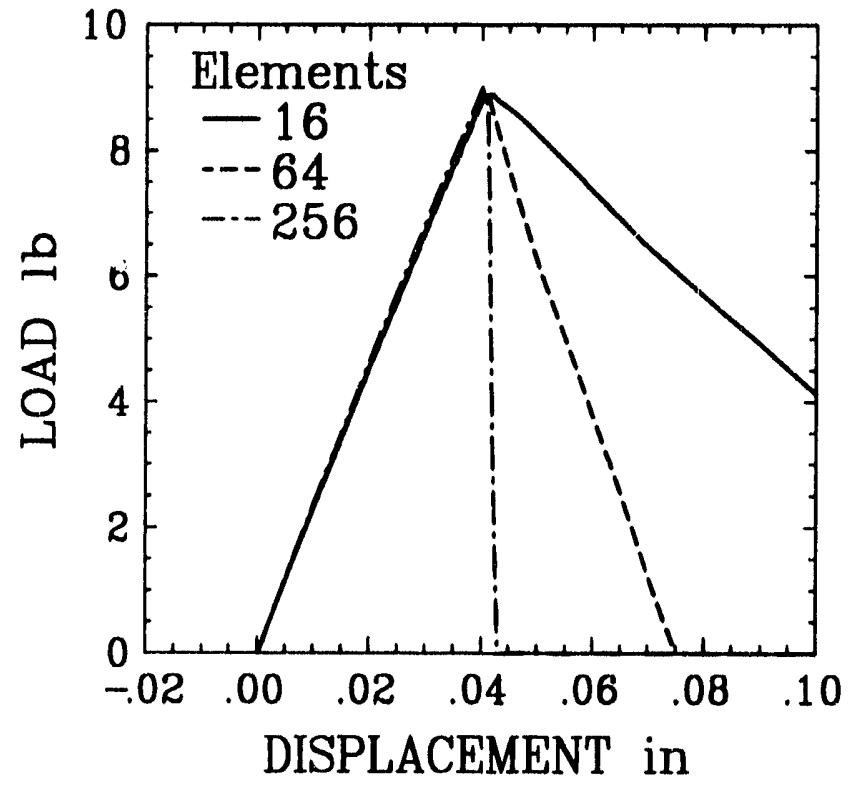

Figure 5.7. Applied Load vs. End Displacement - Principal Stress Plasticity. 


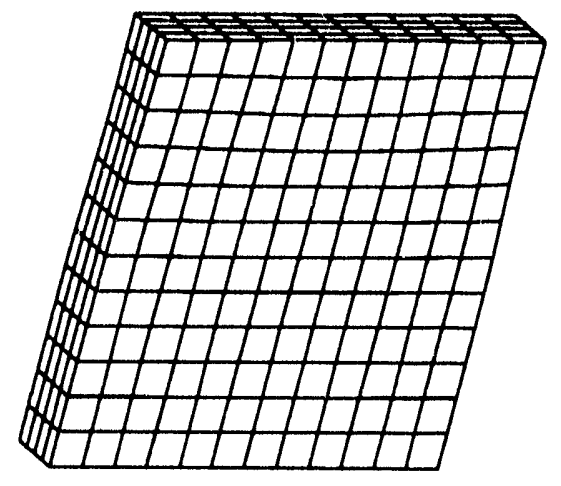

(a) 576 elements
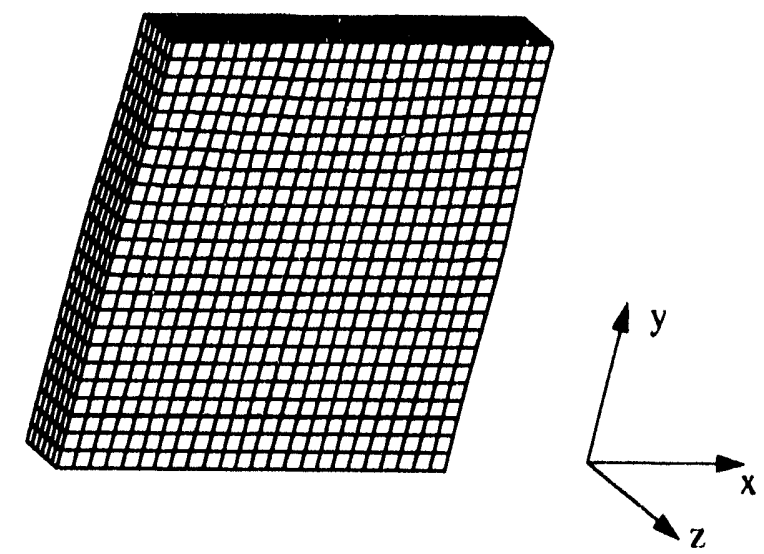

(b) 3456 elements

Figure 5.8. Finite Element Models of a Thin Plate.

$y$-direction shown in Figure 5.8. The deformed shape of the model and contours of ontof-plane displacement are shown in Figure 5.9. The displacement contonrs clearly show the intiation of necked regions oriented at an angle of approximately 60 degrees from the loading axis. which is very close to the orientation predicted by the bifurcation analysis. A second analysis of this problem was then performed using the fine mesh to evaluate the accurary of the coarse mesh solution. A comparison of results from this analysis (Figure $5.10)$ with results obtained with the coarse mesh indicate that the coarse mesh solution is acceptably accurate.

\subsubsection{Thin Plate Subjected to Equal Biaxial Tension}

In the next analysis, the same plate was subjerted to equal biaxial tension in the $x-y$ plane. The deformed shape of this model and contours of ont-of-plane displacement are shown in Figure 5.11. The numerical analysis indicates that bifurcations will not be generated for an elastic perfectly plastic, von Mises material. The theoretical bifurcation analysis performed previonsly indicates that plates subject to equal biaxial tension wonld have to be constructed from a material which exhibits a significant amomnt of strain-softening for bifurcations to occur. Thus, both the bifurcation analysis and the numerical analysis indicate that this material and loading will not generate any general or discontinnous bifurcations.

To simmlate localization phenomena, we would like to be able to obtain solutions that are numerically stable beyond discontinuous bifurcation points. When the numerical procedure fails to converge, we really don't know if a discontinuous bifurcation point 


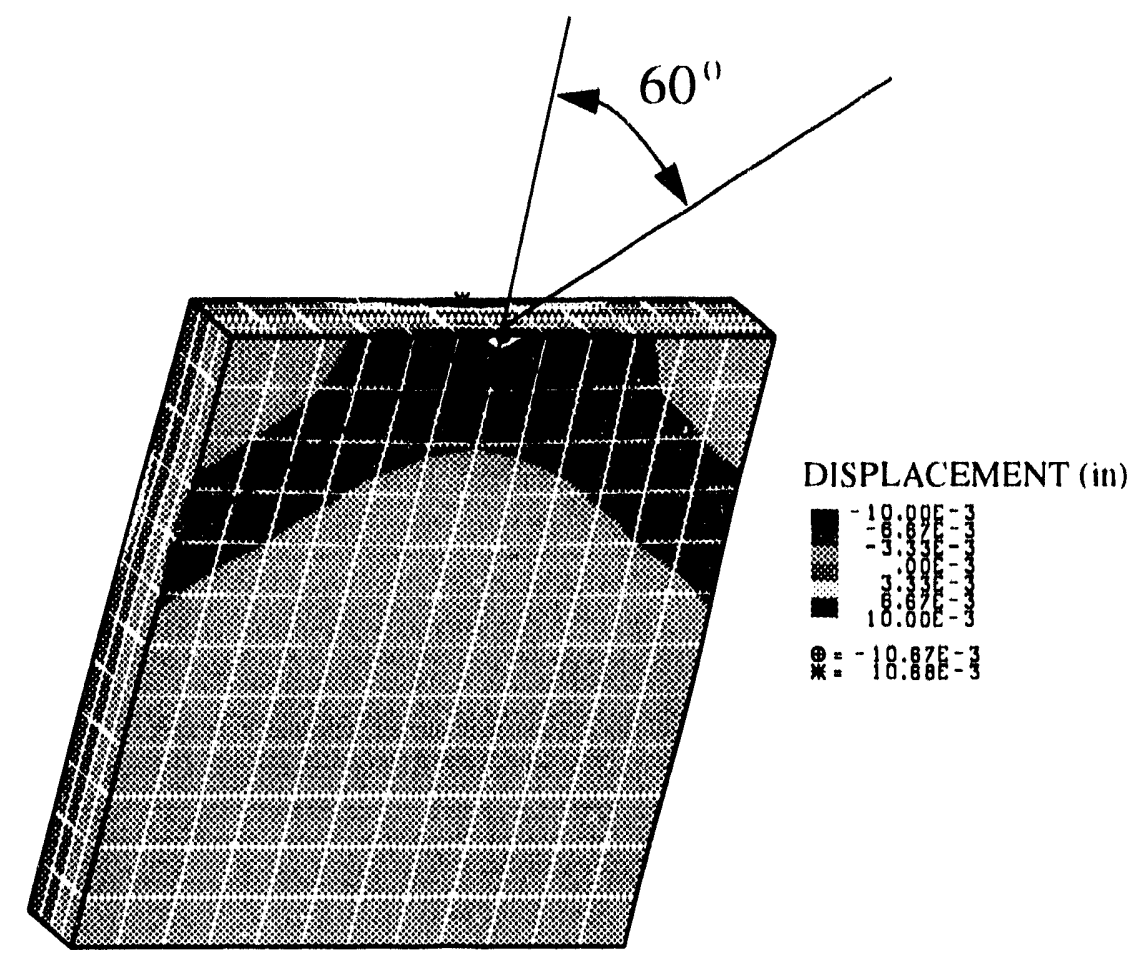

Figure 5.9. Normal Displacement (ontours for a Thin Plate Subjected to Iniaxial Tension - 576 Element, Mesh.

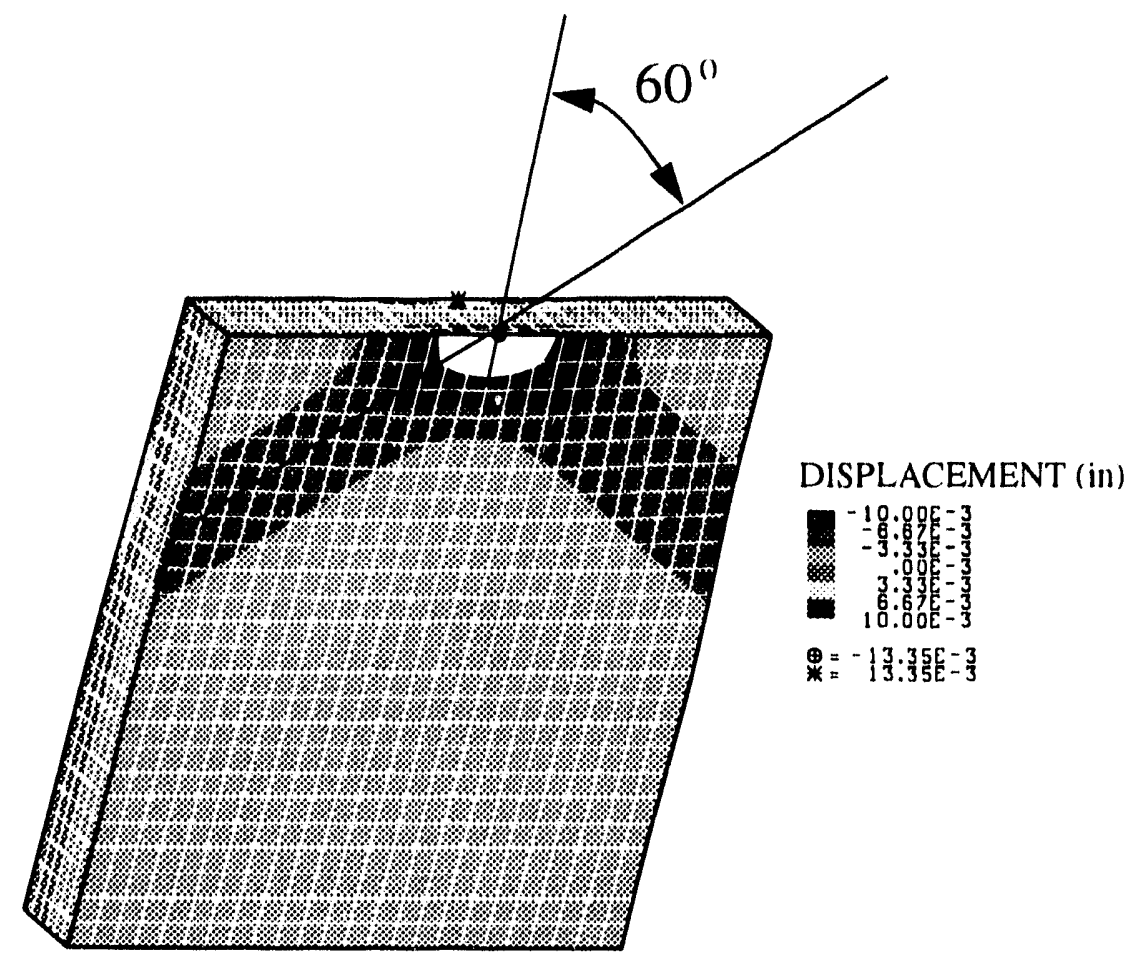

Figure 5.10. Normal Displacement Contours for a Thin Plate Subjected to Uniaxial Tension - 3456 Element Mesh. 


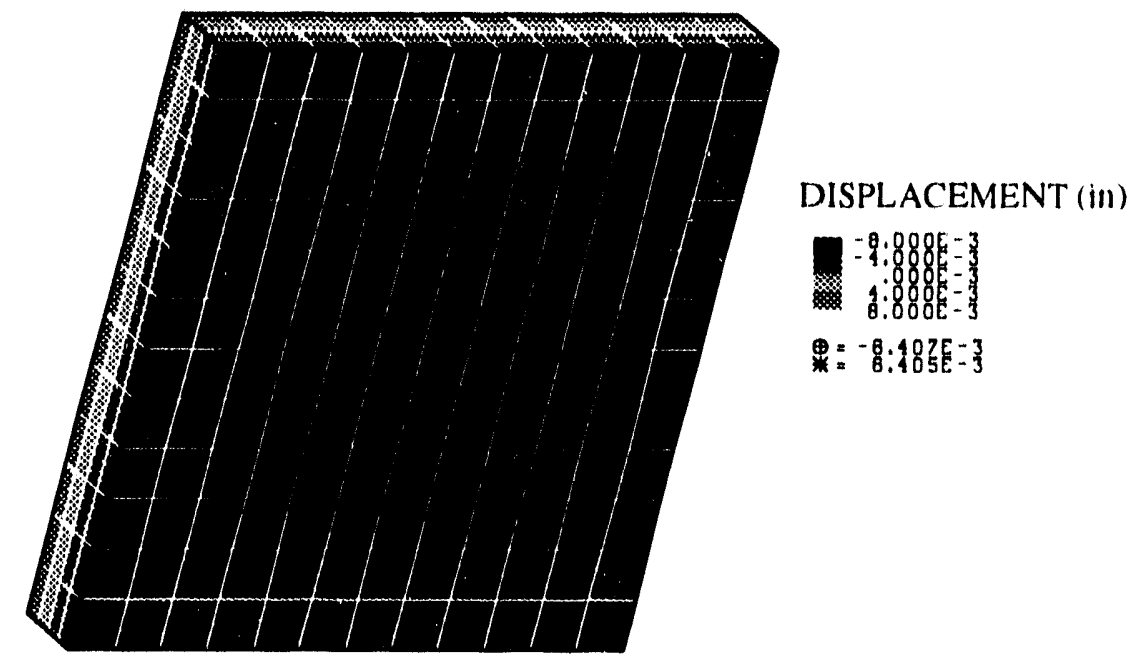

Figure 5.11. Normal Displarement ('ontoms for a Thin Plate Subjected to Equal Biaxial Tension.

has been reached or if the solution algorithm is unstable. To be able to say anything about the initiation and nature of the localization we must be able to obtain convergent, solutions beyond the point of localization. The second problem which must be addressed is that of mesh dependence. To feel comfortahle with solutions to localization problems or any problems we need solutions that converge with mesh refinement. In the next two sertions, we hriefly address these two problems associated with the mumerical simulation of localization phenomena.

\subsection{Modifications to Obtain Numerical Solutions}

Localization phenomena are generally very diffeult to simmlate mumerically. Localization oceurs when the material is softening and the structure is unstable. The load generally does not monotonically increase; thus, it is generally not possible to solve localization problems in which the load is prescribed. In the example problems presented in the previons sertions, solutions were obtained by prescribing displacements which monotonically increase. However in some localization problems, the load-displacement curve exhibits smap-back (Sibreyer and (hen, 1986) which cannot be lollowed by simply prescribing displacements (Figure 5.12). To follow these paths we must resort to more complex procedures for advancing the problem. Riks (1979) and ('risfield (1981) have developed an arc-length control procedure which can solve some structural mechan ics problems exhibiting softening. With this procedure, the solution is incremented a certain distance along the load-displacement curve during each load step and the prescribed loads or displace- 


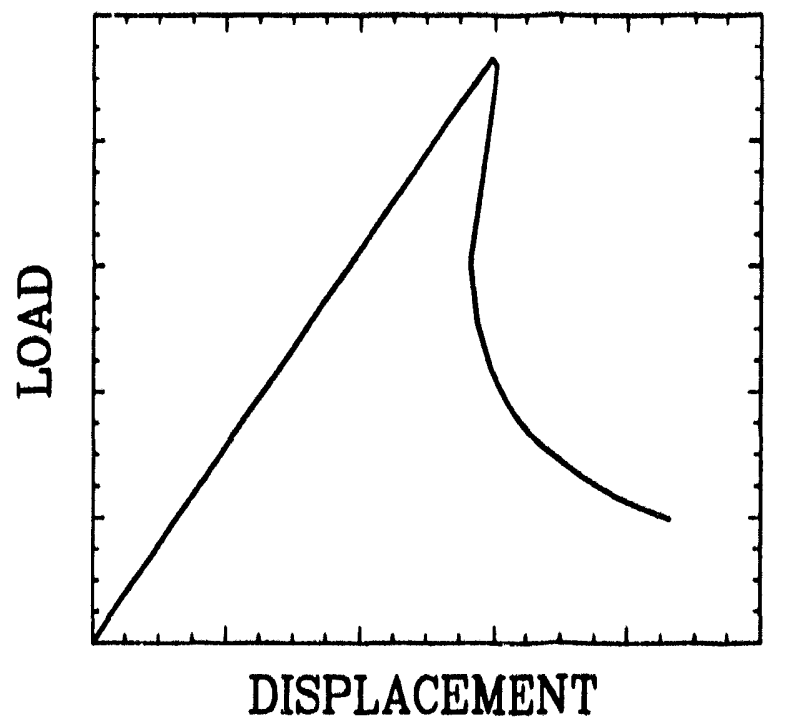

Figure 5.12. Load-Displacement Curve Exhibiting Snap-Bark.

ments are functions of this constraint. However, de Borst (1986) and ('hen, Neilsen and Schreyer (1991) have found that the arc-length control procedure is not robust colongli to obtain solutions to many localization problems. Robustness of the arr-length control procedure is improved significantly by using a norm of the incremental displacements associated with nodes near the localized zone in place of a global norm as the constraint (de Borst, 1986). (hen, Neilsen and Schreyer (1991) propose a solution procedure in which the increment in damage or plastic deformation generated in some critical element is the constraint. With this procedure, the damage or plastic strain increment is prescribed to monotonically increase and the load needed to generate the prescribed increment in plastic strain or damage is computed. This solution procedure has been successfully used in the solution of a number of localization problems.

\subsection{Modifications to Eliminate Mesh Dependence}

Even if the solution algorithm is robust enough to solve the problem, we find that solutions to localization problems are mesh dependent as shown in Section 5.1.2. If the mesh is oriented along the localized zone, the aize of the localized deformation zone will be equal to the dimension of the eloments being used. The first and simplest approach for coping with the mesh dependence is to simply use elements that are the same size as the expected localized deformation zone. With this approach there is really no way to evaluate the accuracy of the solution with mesh refinement because the predicted size of the localized deformation zone is mesh dependent. The second approach is to use non-local constitutive theories which have some internal length scale as a material parameter. When this approach is used, elements which are smaller than the width of the localized deformation zone are usually recuired to capture the localization. The 
third approach is to view localization and the size of the localized deformation zone as a structural property which is independent of the material or constitutive response and to assume that the localized deformation zone is embedded within an element. With this approach, elements which are larger than the width of the localized deformation zone rall he nsed which is appealing because the size of localized deformation zones observed in experiments involving various materials are generally much smaller than the structure being analyzed. Pietruszeak and Mroz (1981), ()rtiz, Leroy and Needleman (1987), Belytscliko, Fish and Engelmann (1988), and Neilsen, ('hen and Schreyer (1991) have lised varions approaches to embed localized deformations zones within elements. Pietruszcak and Mroz (1981) assume that material ontside the localized deformation zone is rigid and that element deformations are governed by deformations within the localized deformation zone. Ortiz, Leroy and Needleman (1987) enhance the shape functions to allow for deformations assoriated with the first possible discontimuons bifurcation. Belytschko, Fish and Engelmann (1988) modify the strain nodal-displacement matrix to account for the existence of the localized deformation zone within the element. With this approanth the standard mapping between clement strains and nodal displacements is replaced by mappings between softening zone strains and nodal displacements and nuloading zone. strains and nodal displacements. Neilsen, (hen and Schreyer (1991) investigate the use of embediled elements with damaging materials. In the next section, we discuss the use of elements with embedded localized deformation zones and reanalyze the problem of Section 5.1.2. Which was severely mesh dependent using standard finite elements.

\subsection{Elements with Embedded Localized Deformation Zone}

('onsider a finite element of length a which is subjected to a muiaxial stress, $\sigma$, (Figure $5.13)$ and constructed from an elastic:-plastic material with the constitutive relation shown in Figure 5.14. When the bar is loaded into the inclastic regime, softening is assumed to occur mniformly in a region of length s that extends throngh the bar (s is a material parameter). Material ontside the softening zone elastically unloads. If the bar is modeled with finite elements and the elements are chosen such that the softening zone is modeled with a single clement, then a conventional finite element approach can be used. 'The difficulty arises when the softening zone is smaller than a single elenent. The constitutive relation in Figure 5.14 describes the material behavior, but camnot completely describe the behavior of an element in which some of the material is strain-softening and the remaining material is elastically moloading.

If the material ontside the softening zone is elastically unloading then the strain rate in the moloading region, $i_{u}$, is given by

$$
\dot{\epsilon}_{u}=\frac{\dot{\sigma}}{E}
$$

where $E$ is the elastic modulus. The strain rate in the softening zone, $\dot{\theta}_{s}$, is as follows:

$$
\dot{i}_{s}=\frac{\dot{\sigma}}{E}+\frac{\dot{\sigma}}{H}
$$




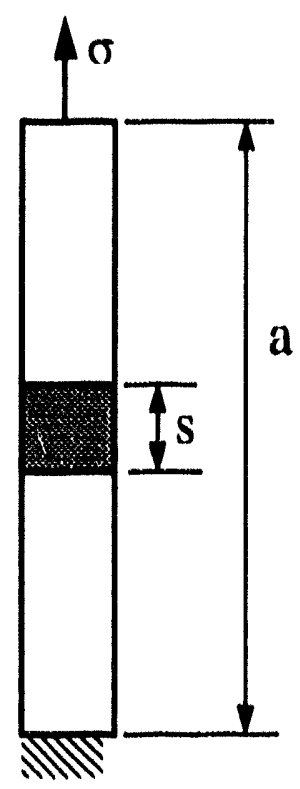

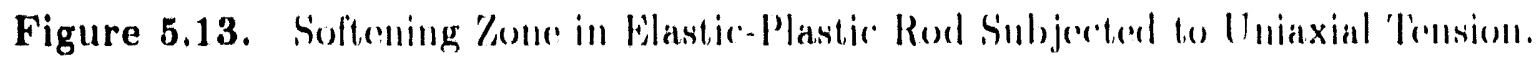

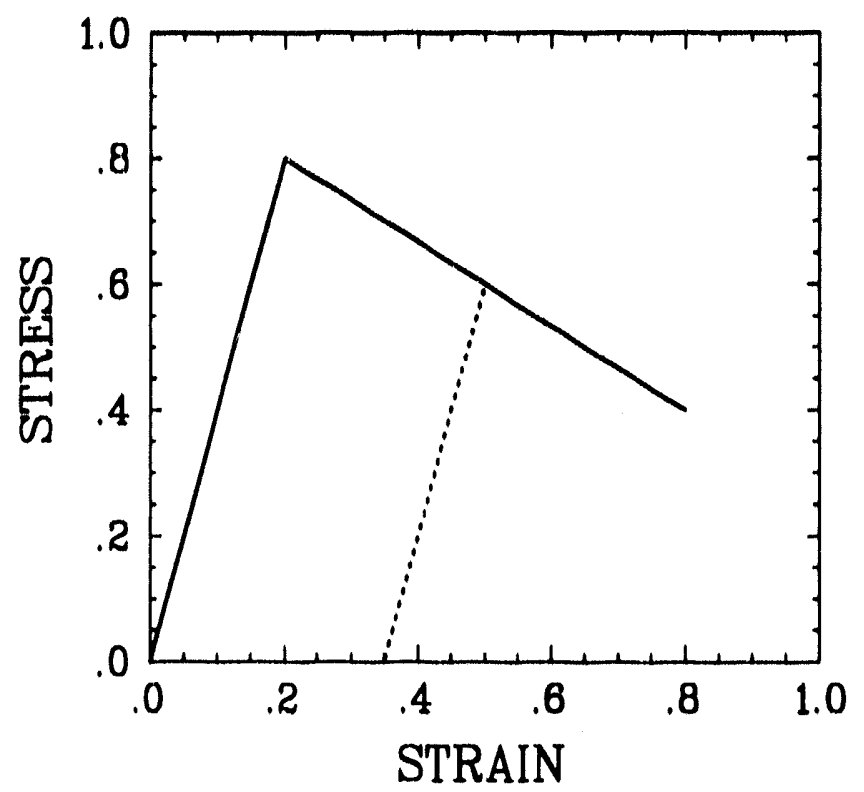

Figure 5.14. Constitutive Relation for Elastic-Plastic: Matcrial. 


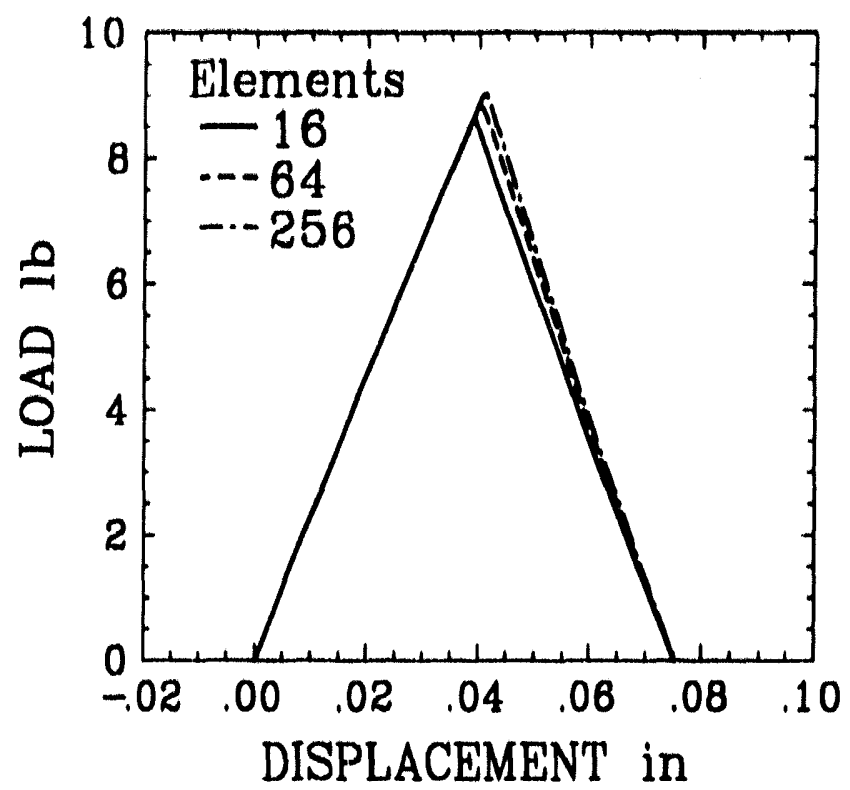

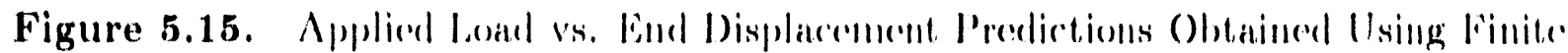

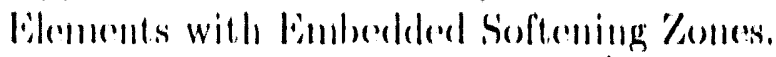

where $/ 1$ is the hardening modulus for the material. 'The average edement strain rate, $i$, is givenll by

$$
i_{1}=\frac{\dot{\sigma}}{l}+\frac{s \dot{\sigma}}{\| l l}
$$

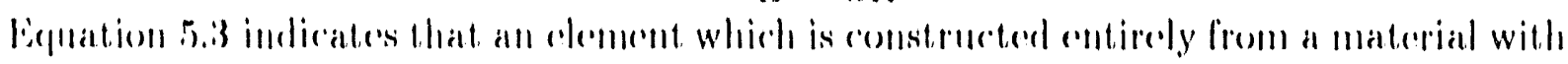
a harderning mordulus, $H_{1}$, in follows:

$$
I_{t}=\frac{11 a}{. s}
$$

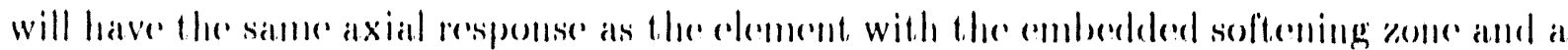

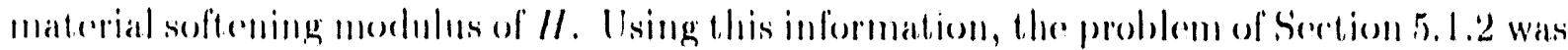
reanalyzed using alemente softening moduli which are mesh dependent. In this antalysis, the material has a softerning modulus of 50 pes and the softering zome is assumed to have

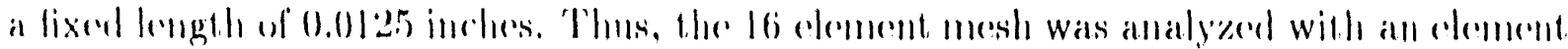

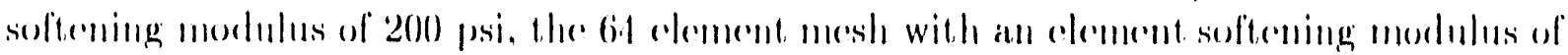

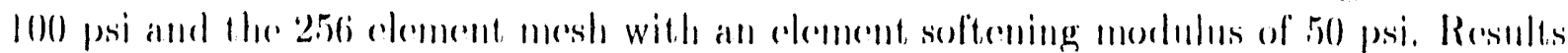

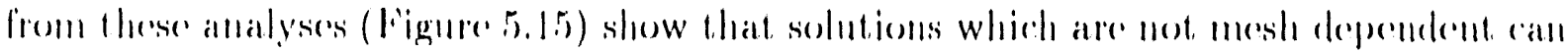
be obtained by assumning that the softening zome hats a fixed size and is conberdeded with hin a single clement.

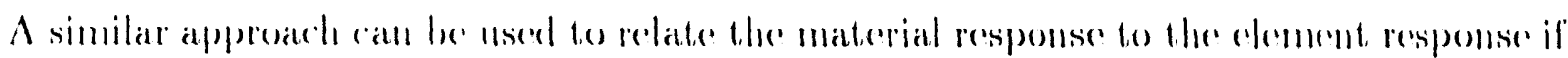
the material is clastic-dannaging with the constitutive relation shown in Figure 5.16. If we assume that material outside the softening zome is not dannaged and is simply elastically monloading, then strain in the muloading region, $c_{n}$, is given by

$$
c_{u}=\frac{\sigma}{E}
$$




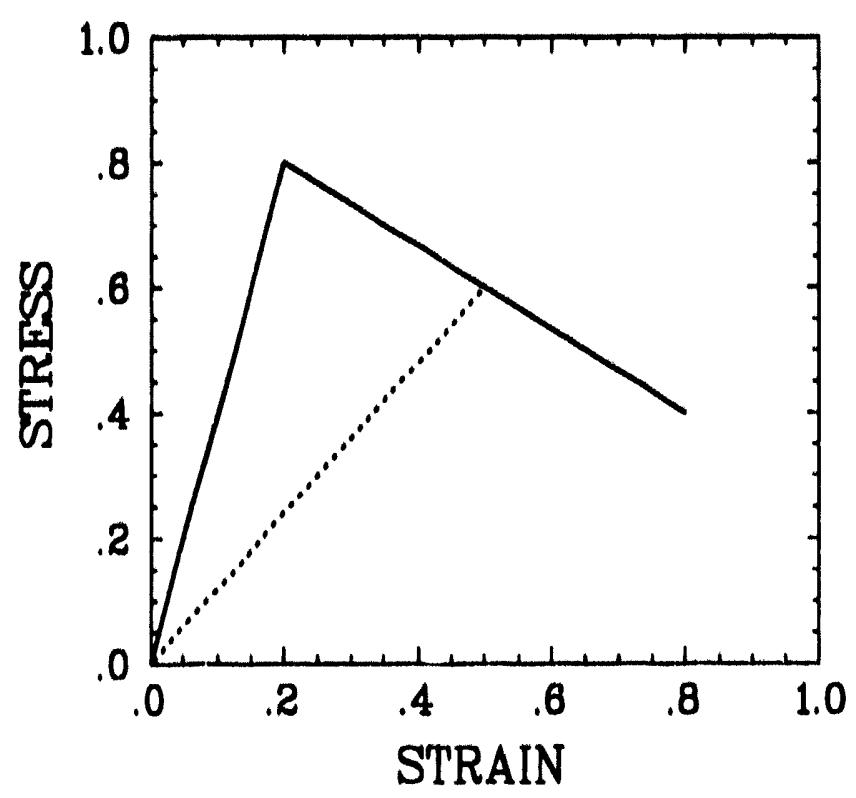

Figure 5.16. ('onstitutive Relation for Elastic-Damaging Material.

Strain in the softening zone, $e_{s}$, is given by

$$
\mathrm{A}=\frac{\sigma}{E(1-w)}
$$

where $w$ is damage in the softening zone material. The following expression for average dement strain, te, can be derived using the two previous ecfuations

$$
c_{c}=\left[1-w+\frac{s}{a} w\right] c_{s}
$$

If the element stress, $\sigma$, and strain, te, are related by

$$
\sigma=E\left(1-w_{\mathrm{r}}\right) \epsilon_{\mathrm{e}}
$$

where $w_{e}$ is the equivalent elenent damage, then it can be shown that the element dannage is related to damage in the softening zone material, $w$, by the following ecfuation.

$$
w_{e}=\frac{s w}{[a-a w+s w]}
$$

As with plasticity, there is a relationship between inelastic material level paranneters and exquivalent inelastic element level parameters. This relationship depends on the size of the softening zone and on the size of the elements being used.

In the example plasticity problem presented in this section, the localized zone was an opening mode oriented across a single row of elements. For general applications in two 
and three dimensions, the embedded localized deformation zone approach must be able to handle both opening and shearing localizations with varions orientations and locations within an element. For example, the localized deformation zone may cross the center of the element or possibly only the corner. It is not clear that the current approaches for alements with embedded localized deformation zones are able to solve general localization problems. Larsson and Runesson (1991) and others have shown that using finite element meshes which are aligned along localized deformation zones significantly enliances our ability to solve general localization problems. Perhaps a combination of adaptive mesh refinement along with embedded localized zone technicues should be considered for the mumerical solution of localization problems. A significant amount of research and development remains to be done in this area before localization problems can be solved routinely. 


\section{A MODEL FOR POLYURETHANE FOAM}

Experimental investigations by Shaw and Sata (1966) and Patel and Finnie (1969) indicate that the initial yield surface for polyurethane foam can be described using a principal stress theory. Recent experiments (Donald and Maji, 1992) indicate that polyurethane loam exhibits localization, plastic deformation and damage when it is crushed. For compressive and tensile loads, the localized deformation zones are oriented in a direction normal to the principal stresses. The bifurcation analyses of principal stress plasticity models indicate that these models do predict the localized deformations exhibited by polyurethane foams. 'Thus, the principal stress plasticity model for polyurethane foam developed by Neilsen, Morgan and Krieg (1987) does capture the localized deformations exhibited by rigid polyurethane foams. 'The original polyurethane foam model along with modificalions that reflect skeleton damage and improved behavior at lock-up are presented in this section.

\subsection{Description of the Foam as a Mixture}

The first step in the development of the constitutive theory for polyurethane foams was an examination of the individual components of the foam structure. Rigid polyurethane foams consists of closed polymer cells with air inside the cells. Therefore, each foam consists of two structural components: (1) the polymer structure or skeleton, and (2) a mixture of air and polymer particles. In applications where the air cannot escape from the skeleton during loading, the air contribution may be significant. For example, in hydrostatic and triaxial compression tests that were performed by Donald and Maji (1992). the samples were jacketed and the air could not escape. Thus, a model which considers the contribution of the air to the overall structural response of the foams is needed to capture the foam behavior exhibited in these tests. Total foam response can be decomposed into the response of the skeleton and the response of the air in series with the polymer wall material as shown in ligure 6.1 .

Since, the air/polymer does not resist any shear deformation, the air/polymer contribution is completely volumetric. For convenience, the skeleton is assuned to occupy the same space as the foam. This implies that the skeleton strain is equal to the foam strain. Also, the foam stress, $\sigma_{f}$, is given by the following equation

$$
\boldsymbol{\sigma}^{f}=\boldsymbol{\sigma}^{s k}-p \mathbf{i}
$$

where $\boldsymbol{\sigma}^{s k}$ is the skeleton stress and pi represents the air/poiymer contribution. The pressure in the nixture of air and polymer particles, $p$, is positive in compression. 'To better understand this equation, consider a hydrostatic rompression test in which the 


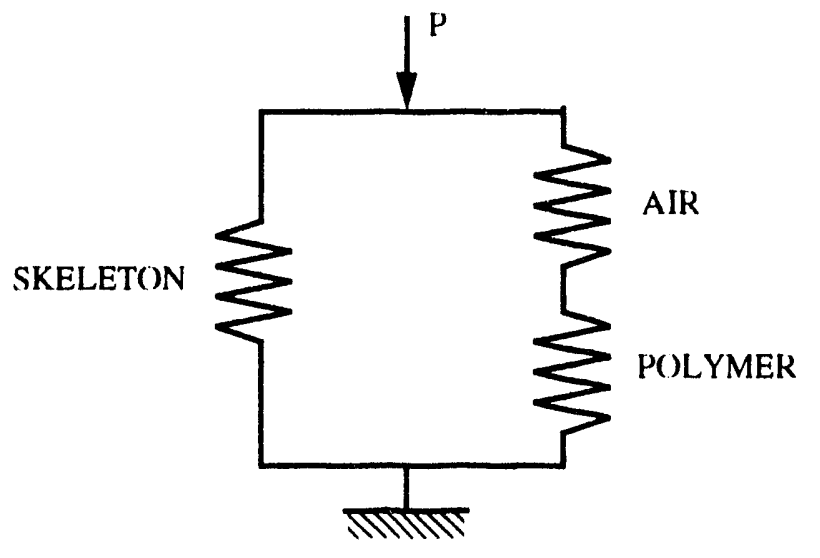

Figure 6.1. Decomposition of Foam Response into Skeleton and Air/Polymer Responses.

foam sample is jacketed and the air is not allowed to escape. If the foam consisted of loosely comnected particles of polymer, then the skeleton contribution to the foam response would be negligible and the external pressure applied to the foam would equal the internal air/polymer pressure. This foam would not be able to resist any deviatoric loading. In most foams, however, the skeleton is structured so that it can carry load and the contribution of the skeleton must be added to the air/polymer contribution to determine how much load the foam can carry. In the next section, an expression for the air/polymer contribution as a function of the foam strain is derived.

\subsection{Air/Polymer Response}

An expression for the air/polymer pressure, $p$, was obtained by assuming that the air trapped within the cells is a perfect gas and that the foam compression is an isothermal process. The isothermal assumption is made becallse the air is in intimate contact with the polymer that makes up the cell walls and the thermal mass of the polymer is significantly larger than the thermal mass of the air. These equations indicate that the perfect gas equation of state can be used for the air and that

$$
p^{a i r} V^{a i r}=p_{o}^{a i r} V_{o}^{a i r}
$$

where $p^{\text {air }}$ is the current air pressure, $V^{\text {air }}$ the current air volume, $p_{o}^{\text {an }}$ the original air pressure, and $V_{o}^{\text {air }}$ the original air volume. The original air volume is related to the original foam volume, $V_{o}$ by

$$
V_{0}^{a i r}=V_{o}^{\prime}(1-\Phi)
$$

where $\Phi$ is the volume fraction of solid material. If we assume that the volume of the polymer is ixed when the foam is manufactured and merely changes its position as the foam deforms, an expression for the enginetering volume strail, $\epsilon_{v o l}$, is

$$
r_{\text {vol }}=\frac{\Delta V^{\text {air }}}{V_{o}}
$$


Prior to loading, we assume that the internal air pressure, $p_{0}^{\text {air }}$, is in equilibrium with the environment, i.e., $p_{0}^{a i r}=14.7$ psi at sea level or $p_{0}^{a i r} \approx 12.5$ psi here in Albuquerque, New Mexico. The change in internal air pressure due to the loading $p=p^{a i r}-p_{o}^{a i r}$ is related to the change in air volume as follows

$$
\Delta V^{a i r}=\frac{-p V_{o}(1-\Phi)}{p_{o}^{a i r}+p}
$$

Combining the above equations we obtain the following expression for the air contribution, $p$, in terms of the current engineering volume strain, $e_{\text {vol }}$

$$
p=\frac{-p_{o}^{a i r} e_{v o l}}{\left(e_{v o l}+1-\Phi\right)}
$$

This equation is used by Neilsen, Morgan and Krieg (1987) in their foam model and also by Gibson and Ashby (1988). In the development of Equation 6.6, a critical assumption is that the volume of the polymer does not change during loading. This assumption is only valid when the pressures are small. If the pressures are large, the volume of the polymer which makes up the cell walls will change according to the bulk modulus of the polymer, $K^{\text {poly }}$, as follows

$$
\Delta V^{\text {poly }}=\frac{-p V_{0} \Phi}{K^{\text {poly }}}
$$

The bulk modulus for polyurethane is approximately 150,000 psi. The expression for engineering volume strain is now given by

$$
e_{v o l}=\frac{\Delta V^{a i r}+\Delta V^{p o l y}}{V_{o}}
$$

Finally, by combining the above equations the following quadratic equation is obtained

$$
\Phi p^{2}+\left[p_{o}^{a i r} \Phi+K^{p o l y}\left(1-\Phi+\epsilon_{v o l}\right)\right] p+e_{v o l} K^{p o l y} r_{o}^{a i r}=0
$$

This equation can be easily solved and only one root is shown to be physically realistic. Plots of air pressure as a function of volume strain using Equations 6.6 and 6.9 are shown in Figure 6.2. This figure clearly shows that the effect of considering the elastic bulk response of the polymer is only significant for large pressures.

If the air is allowed to escape then the response of particle interaction is given by Equation 6.9 with the initial air pressure, $p_{0}^{a i r}$ set equal to zero. With $p_{0}^{a i r}$ equal to zero, Equation 6.9 reduces to

$$
p=0 \quad \text { or } \quad p=-\frac{K^{\text {poly }}}{\Phi}\left(1-\Phi+e_{\text {vol }}\right)
$$

These equations describe two intersecting lines. The response of the polymer when the air is allowed to escape can be approximated by simply prescribing a small value for the initial air pressure as shown in Figure 6.3. 


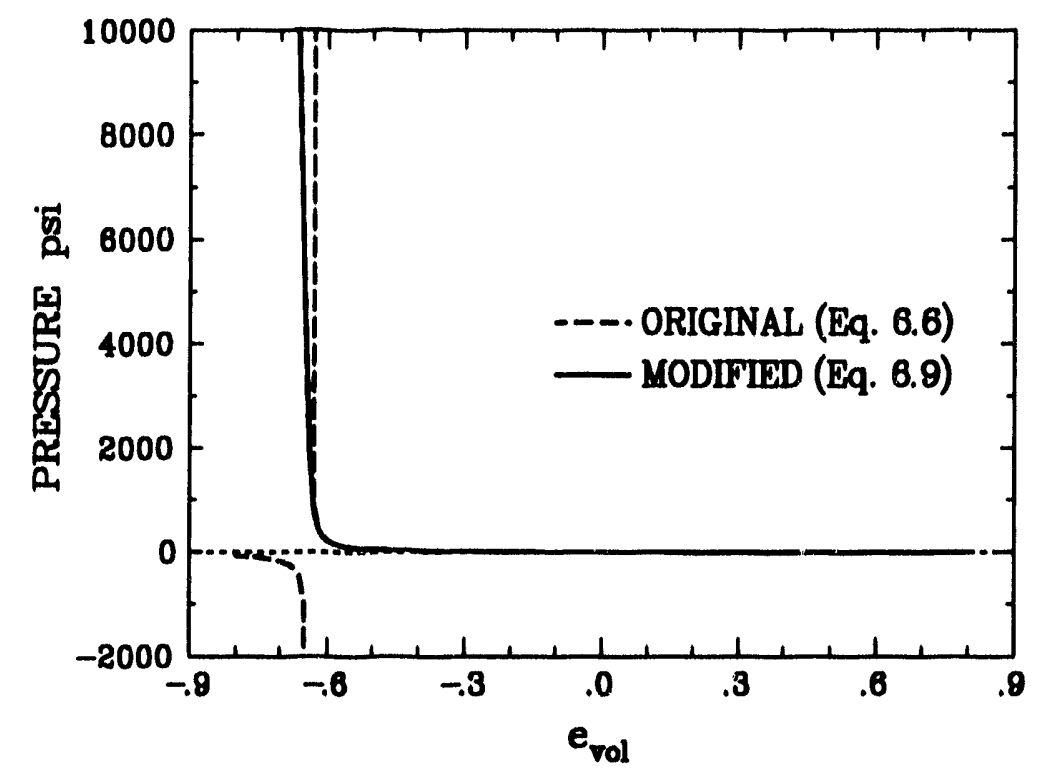

Figure 6.2. Air/Polymer Response for a 20 pcf Polyurethane Foam.

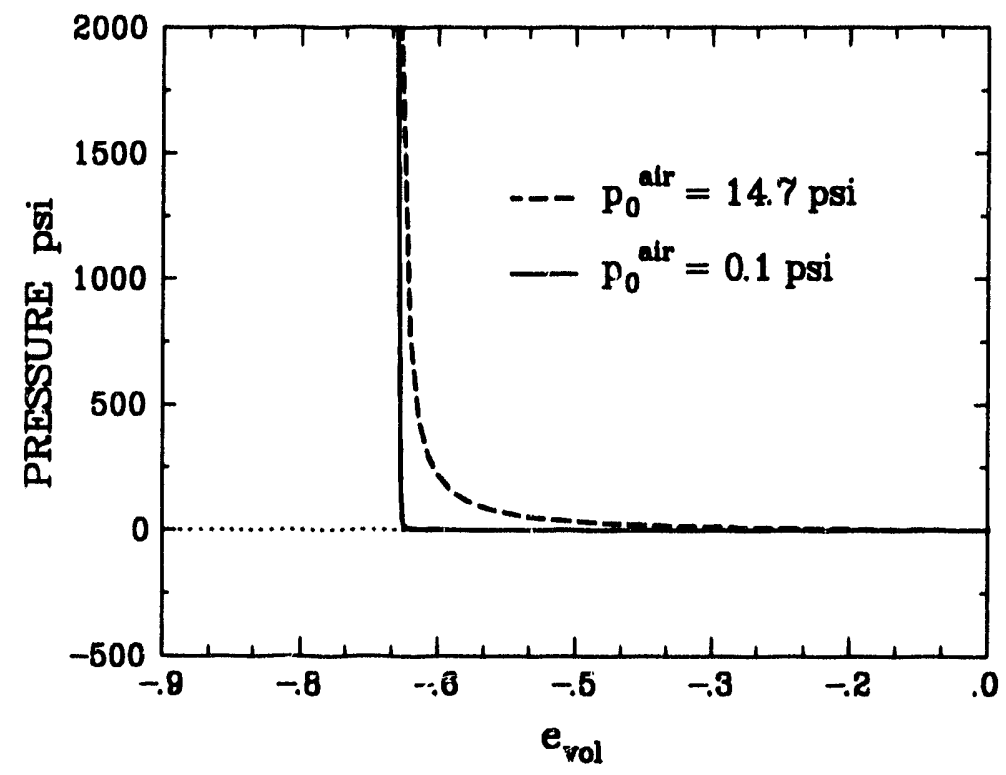

Figure 6.3. Air/Polymer Response for a 20 pef Polyurethane Foam with Air Allowed to Escape. 


\subsection{Plasticity Theory for the Skeleton}

Neilsen, Morgan, and Krieg (1987) developed a plasticity model to describe the contribution of the skeleton to the overall foam response. The yield function for their skeleton model is given by

$$
\Psi^{i}=\frac{1}{2}\left(\boldsymbol{\sigma}: \mathbf{P}^{i}: \boldsymbol{\sigma}\right)^{\frac{1}{2}}-A<I I^{\prime}>+B\left(1+C e_{v o l}\right)
$$

where $\mathbf{P}^{i}$ is the principal projection operator, $I I^{\prime}$ is the second invariant of the deviatoric strains, $<>$ is the Heaviside step function, and $e_{y o l}$ is the engineering volume strain. The yield stress in each principal stress direction is a function of both the volume strain and the deviatoric strain. $A, B$, and $C$ are material constants. The constant $B$ is the yield stress of the skeleton for purely hydrostatic loading, and the product of $B$ and $C$ is the slope of the skeleton volumetric response after yielding for purely hydrostatic loading. The constant $A$ is equal to the difference between the axial yield stress for hydrostatic loading and the axial yield stress for deviatoric loading. In Equation 6.11, the term $\left.A<I I^{\prime}\right\rangle$ is active only if the loading is deviatoric. This yield function was based on a series of uniaxial, hydrostatic and triaxial compression tests on lowdensity rigid polyurethane foams which were completed by the (ivil Engineering Research Facility (CERF) at the New Mexico Engineering Research Institute (NMERI) in 1982. Results from this experimental investigation are documented by Neilsen, Morgan and Krieg (1987).

In triaxial compression tests, the material is subjected to some level of hydrostatic compression and subsequently additional load in one direction only. Results from NMERI's triaxial tests on foam indicated that the foam exhibited a higher strength when the additional axial load was applied as opposed to hydrostatic compression only. The application of additional axial load corresponds to the introduction of deviatoric strains which led to the assumption that the yield surface grows when deviatoric strains are introduced. $\Lambda$ recent series of tests completed by Donald and Maji (1992) reveal that the apparent increase in strength associated with the application of the additional axial load is actinally due to the anisotropic character of the low density foams. During the NMERI tests the additional axial load was always applied in the rise direction of the foam and the foam is generally stronger in the rise direction than in directions perpendicular to the rise direction. Therefore, for the improved model developed in the next section, we adopt an anisotropic yield function that does not include a jump associated with the introduction of deviatoric loading.

\subsection{Coupled Theory for the Skeleton}

Experiments completed by Donald and Maji (1932) indicate that the skeleton exhibits both plastic deformation and damage as it is crushed. Therefore, a coupled plasticity, continuum damage model for the skeleton would be more appropriate than the previously 
developed plasticity model. The experimental evidence suggests that a principal stress based model is appropriate for polyurethane foams; thus, we adopt the following yicld (damage) function

$$
\Psi^{i}=\frac{1}{2}\left(\boldsymbol{\sigma}: \mathbf{P}^{\mathbf{i}}: \boldsymbol{\sigma}\right)^{\frac{1}{2}}-\left(A+B e_{v o l}\right)
$$

where $\mathbf{P}^{i}$ is again the principal projection operator, $A$ and $B$ are material parameters that are dependent on the current amount of damage in the current principal stress orientation. Information about damage is stored in the damaged elastic stiffness tensor, S. The evolution equations for plastic strain and damage are given by

$$
\dot{\epsilon}^{p}=\dot{\rho} \mathbf{P}^{\mathbf{i}}: \boldsymbol{\sigma} \quad \dot{\mathbf{S}}=-\dot{\omega} \mathbf{S}: \mathbf{P}^{\mathbf{i}}: \mathbf{S} \quad \dot{\mathbf{C}}=\dot{\omega} \mathbf{P}^{i}
$$

The evolution equation for plastic strain indicates that plastic strains will be generated in principal stress directions. The damage evolution equation indicates that the compliance of the material will be increased in a direction parallel to the current maximum tensile stress. Even if the skeleton stiffness is isotropic prior to loading, the damage evolution equation indicates that the material will become anisotropic as it is damaged. Since the material stiffness is not isotropic it cannot simply be represented by two scalars and instead the entire stiffness or compliance matrix must be stored. However, by storing the compliance matrix we have implicitly stored information about load history.

To complete this model we need an additional equation that prescribes the relative amounts of damage and permanent strain generated during an inelastic step. The additional equation is given by

$$
M \dot{\rho}=(1-M) \dot{\omega}
$$

In this equation, we allow the magnitude of the non-negative material parameter $M$ to depend on the sign of the principal stress. Specifically, in compression the inelastic behavior is dominated by plasticity, whereas, in tension the inelastic behavior is dominated by damage.

\subsection{Simplified Isotropic Theory for the Skeleton}

Most existing finite element codes were developed using only isotropic plasticity models and generally do not have the architecture required for the easy implementation of anisotropic models. In this section, an isotropic, coupled plasticity damage model which captures at least most of the behavior exhibited by the foam skeleton is developed. This model uses the yield (damage) function given by Equation 6.12 and the flow law for plastic strain increments given by Equation 6.13. However, for the material to remain isotropic, we must adopt an isotropic evolution equation for damage.

If we assume that the skeleton has a Poisson's ratio equal to zero, then the undamaged elastic stiffness tensor for the skeleton, $\mathbf{E}$, is given by

$$
\mathbf{E}=E_{0} \mathbf{I}
$$


where $E_{0}$ is the initial or original elastic modulus and $I$ is the fourth-order identity tensor. Thus, for this model we adopt a simple isotropic damage evolution equation given by

$$
\dot{\mathbf{S}}=-\dot{\omega} E_{0} \mathbf{I}
$$

With this simple scalar damage model, damage history is stored in a single scalar, the damaged elastic modulus, $E=(1-w) E_{0}$.

\subsection{Analysis of Material Characterization Tests}

A series of tests were recently performed in the Civil Engineering Department at the University of New Mexico to characterize the behavior of rigid pulyurethane foams and aluminum honeycombs (Donald and Maji, 1992). In this section, several finite element analyses of the material characterization tests are performed to determine if the constitutive model developed in this chapter will analytically predict the foam behavior which was observed during the experiments.

The constitutive model developed in this chapter was implemented in the finite element code SANTOS (Stone, 1992). In SANTOS, the constitutive relations are expressed in terms of the deformation rate and Cauchy stress in the unrotated configuration (Taylor and Flanagan, 1986). The analyses were performed using SANTOS and the finite element models of axisymmetric material specimens shown in Figure 6.4. The models were subject to uniaxial compression, hydrostatic compression and uniaxial tension. The predicted response of the foam from the finite element analyses was compared with the experimental observations. Properties that are appropriate for a 5 pound per cubic foot foam were used in this investigation (Table 6.1).

In the first analyses, the models were subject to uniaxial compression. A plot of the undeformed and deformed 200 element mesh is shown in Figure 6.5. The deformed shapes show that the model predicts only axial deformation when the material is compressed in the plateau regime and a combination of axial and radial deformation when the material is loaded into the lock-up regime. This deformation behavior is observed experimentally. Mesh refinement had no significant effect on the predicted compressive response of the material. A plot of the applied load versus end displacement predicted by the finite element analyses is compared with the experimental result in Figure 6.6. This plot shows that response predicted by the finite element analyses is qualitatively accurate;

Table 6.1. Material Parameters for a 5 pcf Polyurethane Foam.

\begin{tabular}{|c|c|c|c|c|c|c|c|c|}
\hline $\begin{array}{c}\text { Elastic } \\
\text { Modulus } \\
(\mathrm{psi})\end{array}$ & $\begin{array}{c}\text { Poisson's } \\
\text { Ratio }\end{array}$ & $\begin{array}{c}\mathrm{A} \\
\text { 'omp. } \\
(\mathrm{psi})\end{array}$ & $\begin{array}{c}\mathrm{B} \\
\text { ('omp. } \\
(\mathrm{psi})\end{array}$ & $\begin{array}{c}\mathrm{M} \\
\text { ('omp. }\end{array}$ & $\begin{array}{c}\mathrm{A} \\
\text { Tens. } \\
(\mathrm{psi})\end{array}$ & $\begin{array}{c}\mathrm{B} \\
\text { Tens. } \\
(\mathrm{psi})\end{array}$ & $\begin{array}{c}\mathrm{M} \\
\text { Tens. }\end{array}$ & $\boldsymbol{\Phi}$ \\
\hline 3,000 & 0.0 & 110 & 0 & 0.1 & 110 & -100 & 1.0 & 0.09 \\
\hline
\end{tabular}


however, the amount of crush required to lock-up the foam is smaller than the erush at lock-up predicted by the analyses. (One possible explanation for this discrepancy is that the polymer particles which make up the cell walls actually begin to contact cach other and carry load before the volume fraction of solid material is close to unity. To correct this discrepancy, an additional particle interaction contribution conld be included or the material parameter, $\Phi$, could be modified to accomint for particle interaction.

In the next analyses, the models were subject to uniaxial tension. A plot of the deformed meshes is shown in Figure 6.7. The deformed shapes show the formation of a discontinuous opening mode bifurcation which is also observed in polyurethane foam sampless subject to uniaxial tension. A plot of the applied load versus end displacement predicted by the finite element analyses is compared with the experimental result in Figure 6.8 . Both the loading and unloading responses predicted by the finite element analyses are shown. The behavior predicted during loading in the softening regime is clearly meshdependent. This result was expected because a non-local feature was not incorporated in the current model. As expected, the unloading curves pass through the origin which indicates that the model predicts only damage when the material is subjected to uniaxial tension.

In the final analyses, the models were subject to hydrostatic compression. Plots of the deformed meshes are shown in Figure 6.9. The size of the original undeformed sample is indicated by the dashed lines in Figure 6.9. These analyses predict the generation of rather unusual deformed shapes and localization during hydrostatic compression tests. A bifurcation analysis of this model and stress state indicates that localization is not expected since the fundamental eigentensor for the tangent stiffness tensor is equal to the second-order identity which has no zero eigenvalue. However, if the stress state is not purely hydrostatic due to numerical roundoff then the bifurcation analysis indicates that localization as exhibited by the finite element analyses may occur. Also, recent sturlies by Benallal, Billardon and Geymonat $(1989,1990)$ of instabilities at the boundary of a solid may help to explain these numerical results.

A plot of applied pressure versus engineering volume strain predicted by the finite olement analyses is compared with the experimental result in Figure (6.10. This plot shows that response predicted by the finite element analyses matches the experimental result reasonably well. Even though the deformed shapes were rather mesh dependent the plots of applied pressure vs. volume strain were not mesh dependent. 


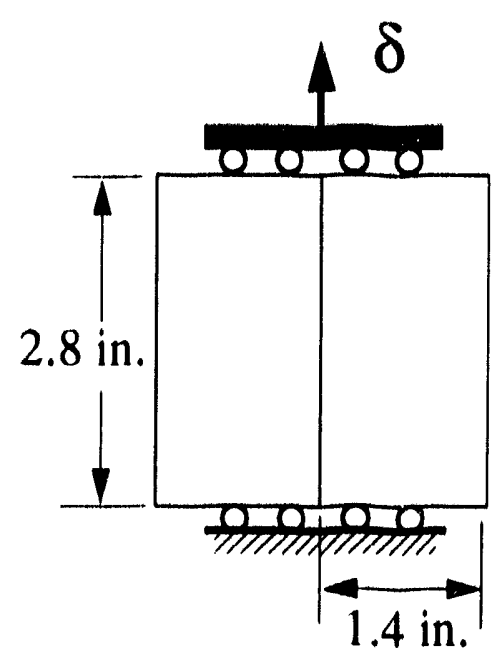

(a) 1 element,

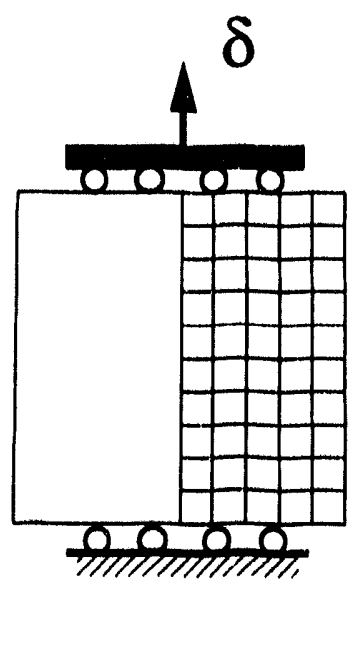

(b) 50 elemonts

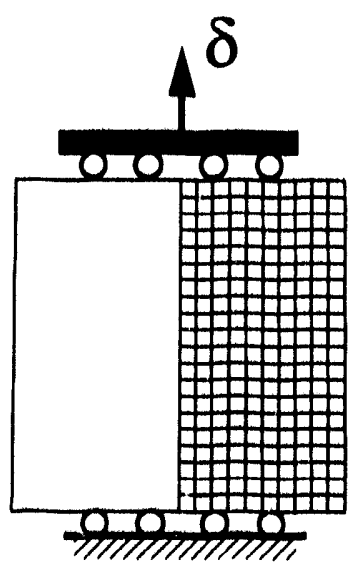

(c) 200 clements

Figure 6.4. Axisymmetric Finite Element Models of Foam Specimens.

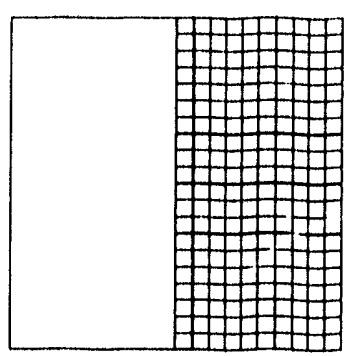

(a) undeformed

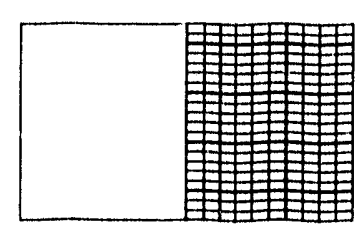

(b) plateau

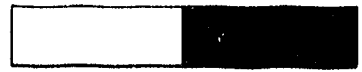

(c) lock-11p

Figure 6.5. Deformed Shape of 200 Element Model Subject to Iniaxial Compression. 


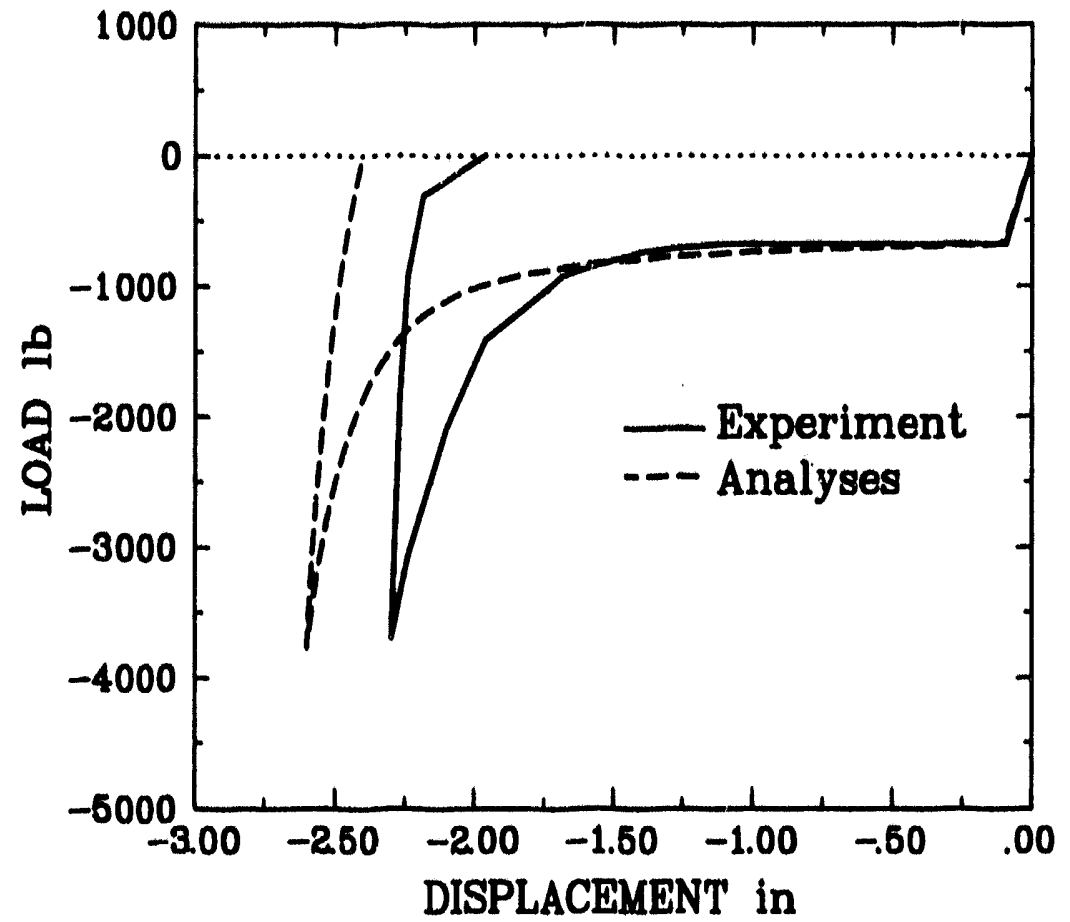

Figure 6.6. Applied Load vs. End Displacement - Uniaxial Compression Comparison of Finite Element Analyses Predictions with Experimental Observations.

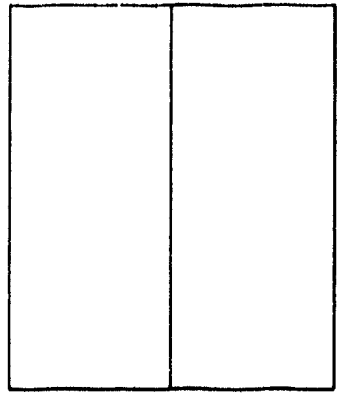

(a) 1 element

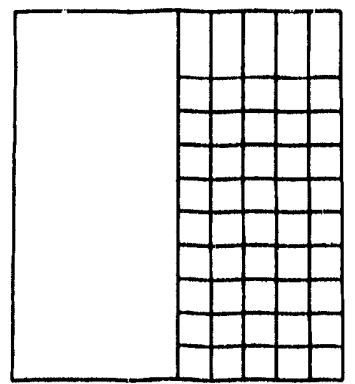

(b) 50 elements

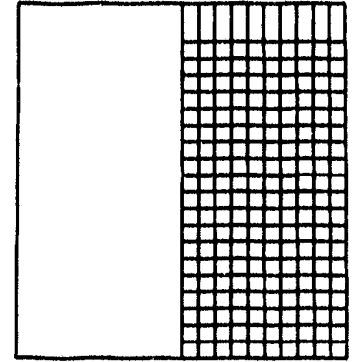

(c) 200 elements

Figure 6.7. Deformed Shape of Models Subject to Uniaxial 'Tension. 


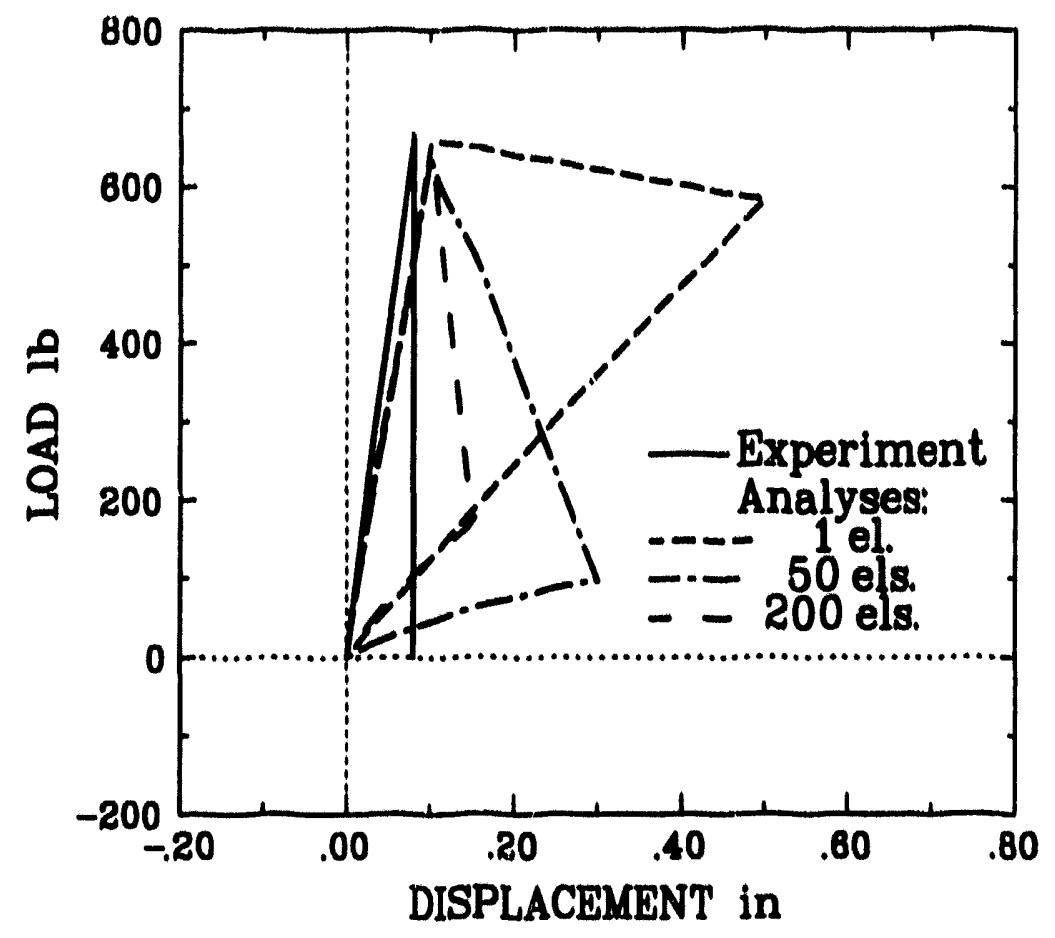

Figure 6.8. Applied Load vs. End Displacement - Uniaxial Tension - Comparison of Finite Element Analyses Predictions with Experimental Observations.

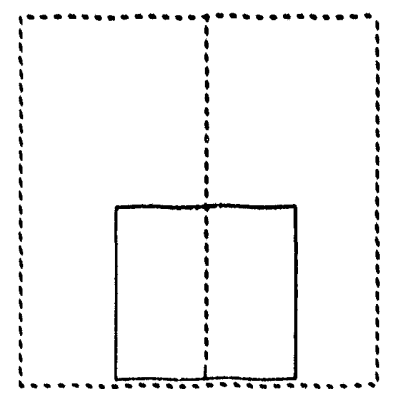

(a) I element

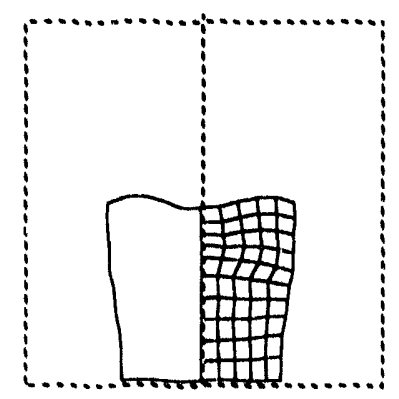

(b) 50 elements

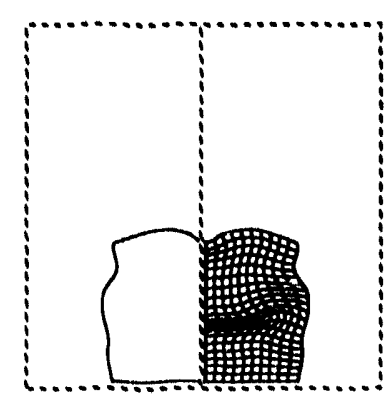

(c) 200 elements

Figure 6.9. Deformed Shape of Models Sinbject to Hydrostatic (ompression. 


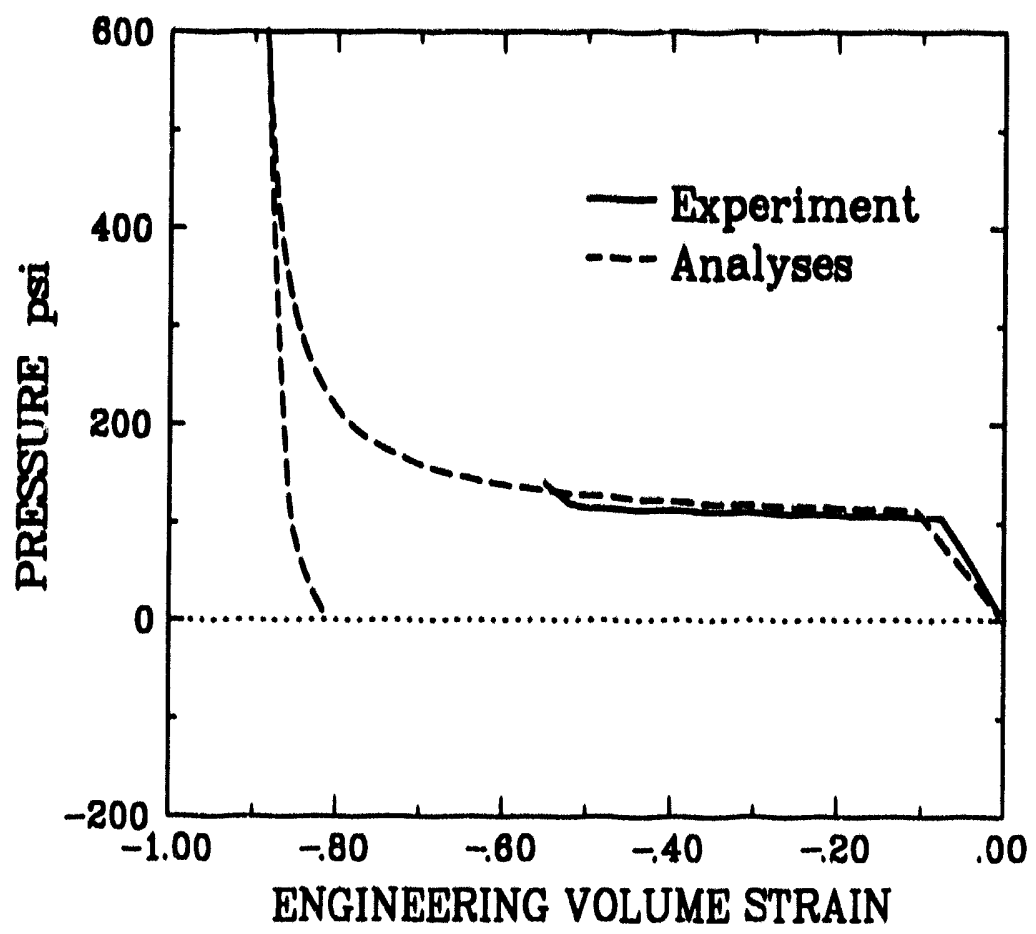

Figure 6.10. Applied Pressure vs. Engineering Volume Strain - Hydrostatic ('ompression - ('omparison of Finite Element Analyses Predictions with lixperiment. (Observations. 


\section{ALUMINUM HONEYCOMB MODEL}

Experiments on alumi:num honeycomb indicate that localization and plastic deformation is generated when the material is crushed. Thus, a plasticity model developed for aluminum honeycomb should predict localization when the material is loaded into the platean regime. The localization exhibited by honeycomb is oriented along geometric lines which is not a typical characteristic of other materials. The only existing model that predicts localizations which are similar to those observed in honeycomb is the MolirCoulomb model which predicts localization with an orientation which is dependent on the internal friction angle and the orientation of the principal stresses. A plasticity model which predicts localization with an orientation that is dependent on the geometry will require a yield function that is dependent on the geometry. Such a model was developed by Klintworth and Stronge (1988). Their model along with a bifurcation analysis is presented in the next section.

\subsection{In-plane Response}

A number of researchers have investigated the in-plane behavior of aluminum honeycomb (Klintworth and Stronge, 1988; (iibson and Ashby, 1988) and developed expressions for the initial yiedd surfaces for this material. Klintworth and Stronge (1988) have investigated the response of aluminum honeycomb subjected to in-plane stresses as shown in lignere 7.1 . Their investigation indieates that the yiold surface for in-plane loads consists of a fimbily of interserting yichl surfiaces with yield functions given by

$$
\psi=u \sigma_{x \cdot r}+b \sigma_{y y}+c\left(\sigma_{x y}+\sigma_{y, r}\right)-i
$$

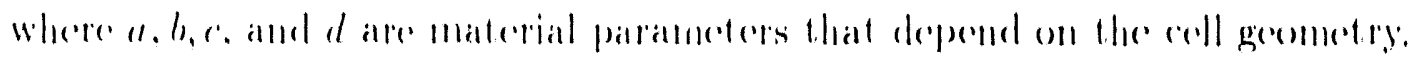

The experimental compression tests indicate that the yield surfaces de not change sig-

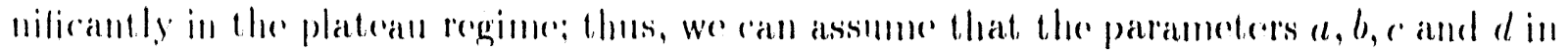
the above yichel functions are constant in the platean regime. Furthermore, if we assume the material exhibits associated flow then

$$
\mathbf{f}=\mathbf{g}=\frac{\partial \Psi}{\partial \boldsymbol{\sigma}} \Rightarrow\left[\begin{array}{lll}
a & c & 0 \\
c & b & 0 \\
0 & 0 & 0
\end{array}\right]
$$

The bifurcation analyses of plasticity theories with associated flow rules indicate that the first possible bifurcation orcurs at the limit point. The mode associated with this bifurcation is characterized by the normal to the yield surface, $f$, which is dependent on the 


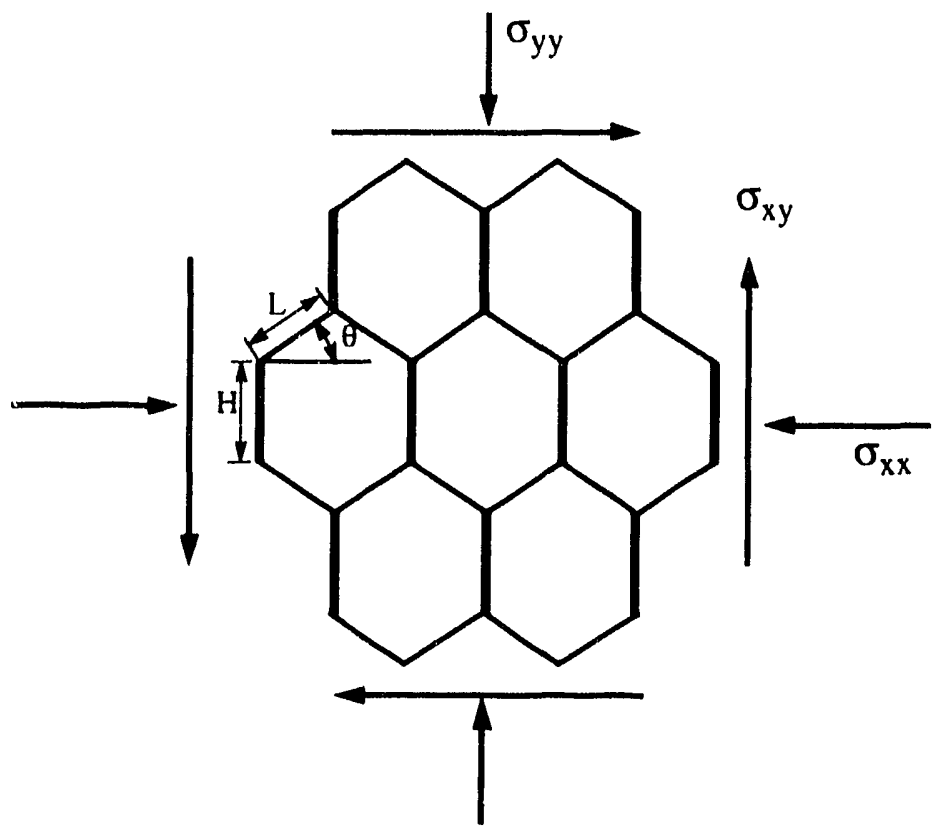

Figure 7.1. In-plane Loading of Aluminum Honeycomb ('ells.

material parameters and thus cell geometry. For example, when the honeycomb is subjected to uniaxial compression in the $x$ direction, the active yield surface is characterized by

$$
\begin{aligned}
& a=-\frac{1}{\Sigma_{x}}-\frac{1}{\Upsilon_{x}} \\
& b=-\frac{1}{\Sigma_{y}}+\frac{1}{\Upsilon_{y}} \\
& c=-\frac{1}{2 \Sigma_{x y}}+\frac{1}{2 \Upsilon_{x y}} \\
& d=1
\end{aligned}
$$

where $\Sigma_{x}, \Sigma_{y}$ and $\Sigma_{x y}$ are elastic buckling parameters and $\Upsilon_{x}, \Upsilon_{y}$ and $\Upsilon_{x y}$ are plastic collapse parameters defined as follows:

$$
\begin{aligned}
\Sigma_{x} & =\frac{\pi^{2} E t^{3}}{12 L^{2}(H+L \sin \theta) \cos \theta} \\
\Sigma_{y} & =\frac{\pi^{2} E t^{3}}{12 L^{3} \cos \theta \sin \theta} \\
\Sigma_{x y} & =\frac{\pi^{2} E t^{3}}{12 L^{2}(L+H \sin \theta)} \\
\Upsilon_{x} & =\frac{t^{2} \sigma_{y}}{2 L(H+L \sin \theta) \sin \theta} \\
\Upsilon_{y} & =\frac{t^{2} \sigma_{y}}{2 L^{2} \cos ^{2} \theta}
\end{aligned}
$$




$$
\Upsilon_{x y}=\frac{t^{2} \sigma_{y}}{2 H L \cos \theta}
$$

where $L, H, \dot{i}$, and $\theta$ are the dimensions and angle that describe a typical cell as shown in Figure 7.1. $E$ is the elastic modulus for the solid material and $\sigma_{y}$ is the yield strength for the solid material. Parameters for a typical aluminum honeycomb are given in Table 7.1 .

Table 7.1. Parameters for a 0.125-5052-.002 Aluminum Honeycomb.

\begin{tabular}{|c|c|c|c|c|c|}
\hline $\begin{array}{c}E \\
\text { psi }\end{array}$ & $\begin{array}{c}\sigma_{y} \\
\text { psi }\end{array}$ & $\begin{array}{c}H \\
\text { in. }\end{array}$ & $\begin{array}{c}L \\
\text { in. }\end{array}$ & $\begin{array}{c}t \\
\text { in. }\end{array}$ & $\begin{array}{c}\theta \\
\text { degree }\end{array}$ \\
\hline $10.0 \mathrm{E} 6$ & 24000 & 0.072 & 0.072 & 0.002 & 30 \\
\hline
\end{tabular}

Using these material parameters we obtain the following values for the yield function constants: $a=-0.0884 \mathrm{in}^{2} / \mathrm{lb}, b=0.0785 \mathrm{in}^{2} / \mathrm{lb}, c=0.0425 \mathrm{in}^{2} / \mathrm{lb}$. By substituting these constants into the expression for $\mathbf{f}$, Equation 7.2, and performing the bifurcation analysis we find that this plasticity model predicts localization at the limit point with an orientation of 30.0 degrees which is very close to the experimentally observed orientation (Figure 7.2.) Furthermore, the orientation of $\mathbf{m}$ indicates that the bifurcation is primarily a shearing type bifurcation which is also consistent with experimental observations.

When the foam is subjected to uniaxial compression in the $y$ direction, the active yield surface is characterized by

$$
\begin{aligned}
& a=-\frac{1}{\Sigma_{x}}+\frac{1}{\Upsilon_{x}} \\
& b=-\frac{1}{0.346 \Phi_{y} \Sigma_{y}}-\frac{1}{\Upsilon_{y}} \\
& c=0 \\
& d=1
\end{aligned}
$$

where

$$
\Phi_{y}=\frac{L \sin \theta}{H}
$$

Using the material parameters given in Table 7.1 we obtain the following yield function constants: $a=0.0736 \mathrm{in}^{2} / \mathrm{lb}, b=-0.0952 \mathrm{in}^{2} / \mathrm{lb}, c=0.0 \mathrm{in}^{2} / \mathrm{lb}$. By substituting these constants into the expression for $\mathbf{f}$ and performing the bifurcation analysis we find that this plasticity model predicts localization at the limit point with an $\mathbf{n}$ oriented at an angle of 48.7 degrees from the $x$ axis which is again reasonably close to the orientation which is observed experimentally (Figure 7.3).

These bifurcation analyses indicate that the plasticity model developed by Klintworth and Stronge (1988) does capture the localized deformations for the load cases that were analyzed. It was beyond the scope of this paper to evaluate all of the yield surfaces required to describe all possible load paths. 


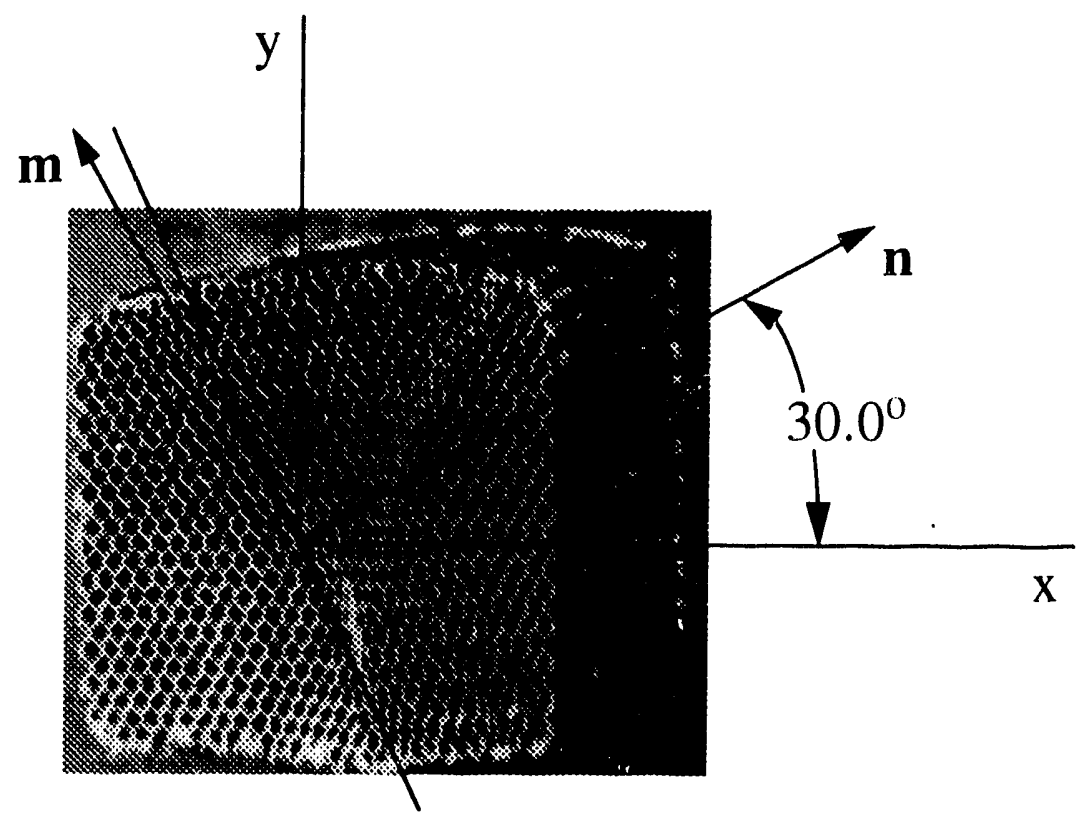

Figure 7.2. Comparison of Bifurcation Analysis Predictions with Experimental Observations of Localization in Aluminum Honeycomb Subjected to Uniaxial Compression in the $\mathrm{x}$-Direction.

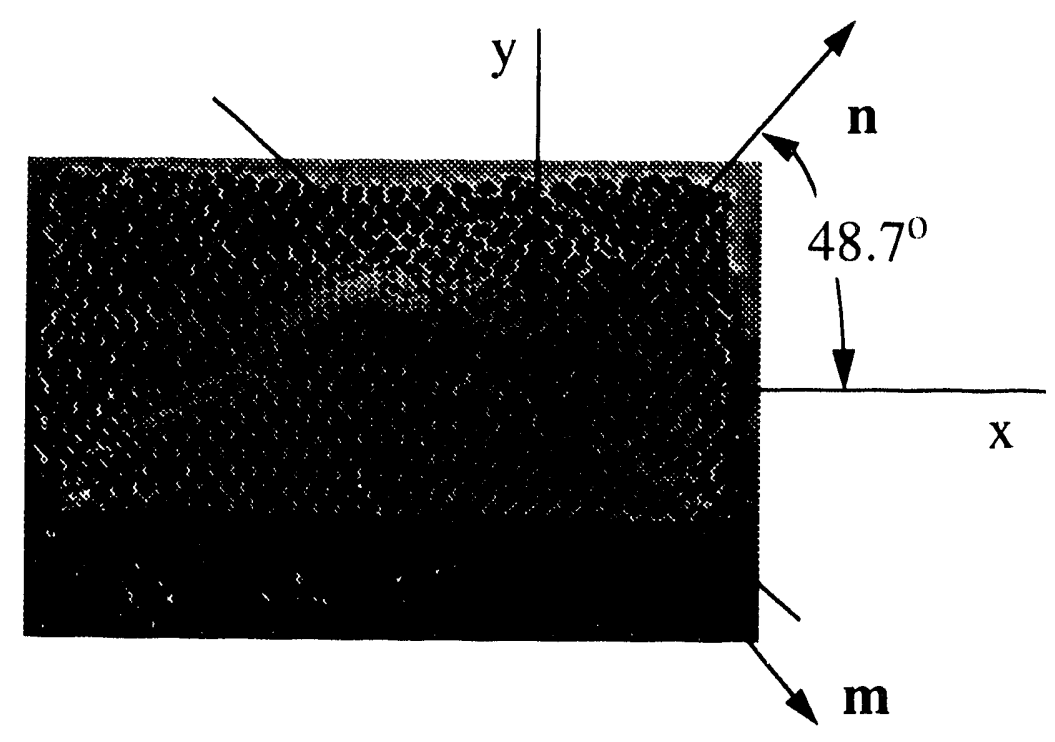

Figure 7.3. Comparison of Bifurcation Analysis Predictions with Experimental Observations of Localization in Aluminum Honeycomb Subjected to Uniaxial Compression in the $y$-Direction. 


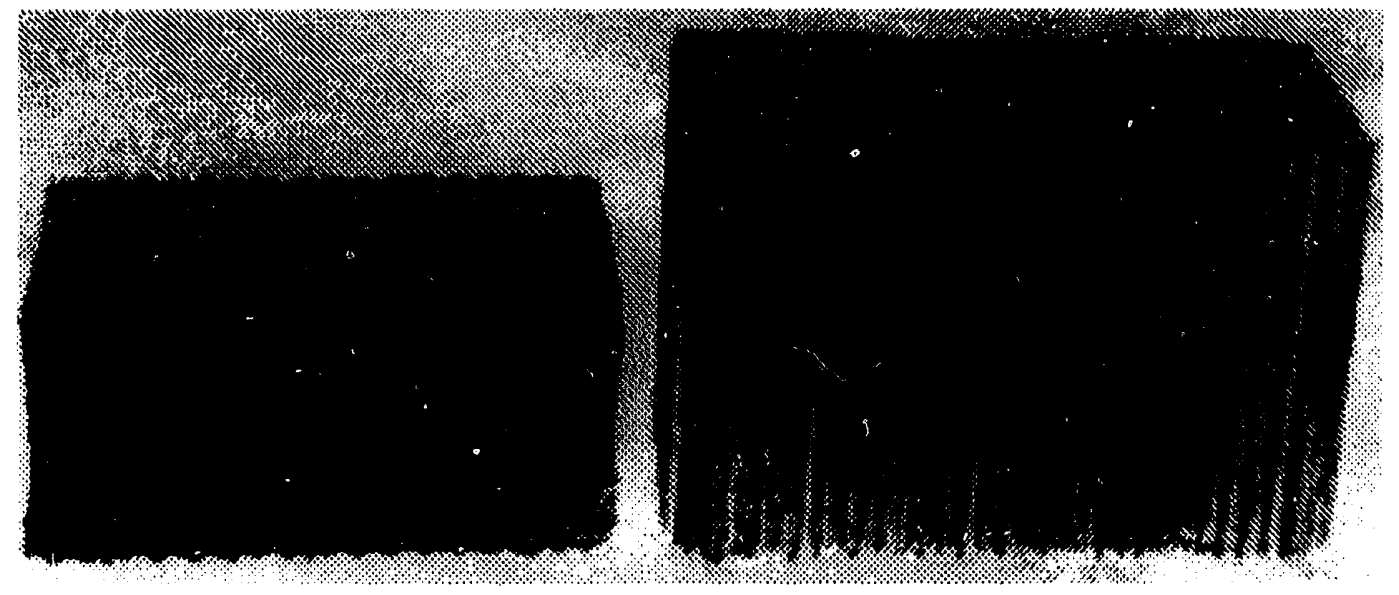

Figure 7.4. Localized Deformation Exhibit by Aluminum Honeycomb Subjected to Uniaxial Compression Along its Cenerator Axis.

\subsection{Generator Axis Response}

When aluminum honeycomb is used in impact limiters the material axes are usually oriented such that the material will be crushed along its generator axis during an accidental impact event. This material exhibits a significantly higher strength when it is loaded along its generator axis as opposed to in-plane. Uniaxial compression tests along the generator axis (Donald and Maji, 1992) indicate that this material exhibits localized deformation oriented in a direction normal to the loading axis when it is loaded into the plateau regime (Figure 7.4). This localized deformation can be captured with an associated flow law and a yield function which uses the principal projection operator, $\mathbf{P}^{z}$, as follows

$$
\Psi^{z}=\boldsymbol{\sigma}: \mathbf{P}^{z}: \boldsymbol{\sigma}-\epsilon\left(\epsilon, \epsilon^{p}\right)
$$

The bifurcation analysis of such a plasticity model presented in Section 4.6.4 indicates that this model will predict localization oriented normal to the principal stress direction at the limit point.

\subsection{A Baseline Model}

The analyses in the previous sections indicate that the rather complex combination of yield functions developed by Klintworth and Stronge (1988) would capture the localized deformations that are generated when the honeycomb is subjected to in-plane loads. In most impact limiter applications, the honeycomb is oriented such that the primary loads are oriented along the generator axis and the material is expected to absorb energy by crushing along the generator axis; thus, to simulate these impact events the most 


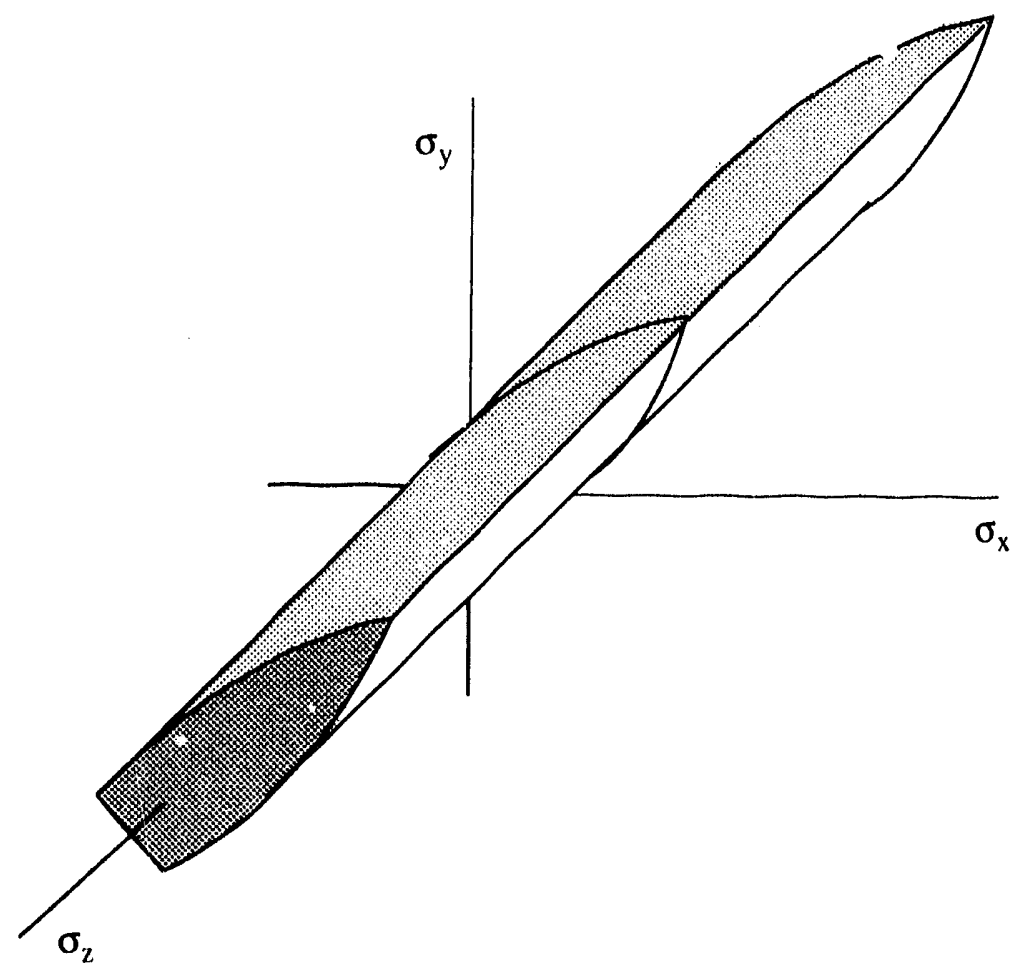

Figure 7.5. Yield Surface for Aluminum Honeycomb.

important behavior that must be captured is the crush of the material along the generator axis. A yield function which uses the principal projection operator, Equation 7.7, is able to predict the localization which occurs when the material is subjected to uniaxial compression along the generator axis. By using this yield function to describe planar caps for the yield surface described by Klintworth and Strong's (1988) in-plane yield functions, we obtain the yield surface shown in Figure 7.5. 


\section{FINITE ELEMENT ANALYSIS OF AN IMPACT EVENT}

A typical impact problem was analyzed using the two-dimensional finite element model shown in Figure 8.1 and the code PRONTO-2D (Taylor and Flanagan, 1986). This plane strain model represents an infinitely long steel cylinder surrounded by a foam layer which is covered with a thin aluminum shell. Three analyses were performed using different constitutive theories for the foam material to investigate the effects of the foam constitutive theory on the predicted response of the impact limiter.

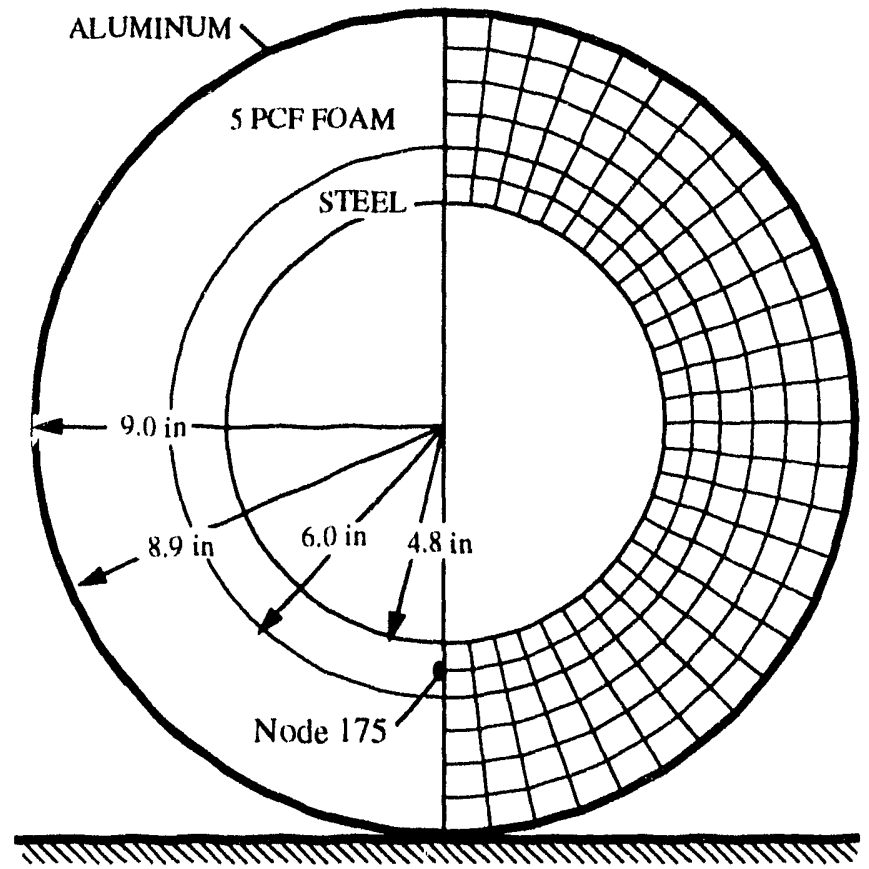

Figure 8.1. Finite Element Model Used in Dynamic Analyses.

The package was dropped onto a rigid surface at an initial velocity of 528 inches per second, and the resulting deformations and accelerations were computed. The impact velocity of 528 inches per second results from a regulatory drop of 30 feet onto an unyielding target for nuclear waste shipping containers.

Material properties used in these analyses are given in Table 8.1. Properties typical of a 5 pound-per-cubic-foot rigid polyurethane foam were used for the foam layer. In the 
first analysis, the foam layer was modeled using a von Mises plasticity model for which properties were determined based on results from uniaxial compression tests on the foam. For this model, the material was assumed to be elastic-perfectly plastic and was given a yiedd strength equal to the platean stress for the foam. In the second analysis, the foam was modeled using the original foam plasticity model developed by Neilsen, Morgan and Krieg (1987). In the final analysis, the foam was modeled using the new theory consisting of plasticity compled with isotropic continum damage. Results from both the miaxial and the hydrostatic tests were used to determine material properties for the foam plasticity and new coupled models.

Results from these analyses are shown in Figures 8.2 to 8.6. Displaced shapes of the finite element model at maximum crush-up are shown in Figure 8.2. When the von Mises plasticity model (Figure 8.2a) is used for the foam material, the foam is not able to exhibit permanent volume changes and is forced to try to flow from beneath the impacting cylinder. $O_{n}$ the other hand, when the original foam plasticity model or the new foam model are used, the permanent volume changes exhibited by foam are captured (Figures $8.2 \mathrm{~b}$ and $8.2 \mathrm{c}$ ).

The predicted vertical displacement, velocity and acceleration of Node 175 (see Figure $8.1)$ in the steel cylinder is plotted as a function of time from initial impact in Figures 8.3, 8.4 and 8.5. The new coupled theory predicts a slightly larger amount of foam crush and a higher rebound velocity than the original plasticity theory. The acceleration time histories generated using the original foam plasticity model and the coupled foam model are very similar. An accurate prediction of the acceleration time history is very important as it provides a measure of the amount of protection provided by the impact. limiter. The displacement, velocity and acceleration predictions generated using the von Mises plasticity theory are significantly different than the predictions gencrater with either of the foam theories. When the foam is modeled with a von Mises plasticity theory, it is not allowed to exhibit any permanent volume changes and unrealistic foam behavior is predicted. Thus, the von Mises theory predicts a peak acceleration level and a rehomal velocity that is moch higher than that predicted by either foam theory.

Finally, a plot of damage in the foam material at maximum crush-1up is shown in Fignre 8.ti. This plot shows that the largest amount of foam damage is generated in the foam material mear the sides of the steel cylinder and not between the steel cylinder and the impact surface. Damage occurs here because the originally circular impart limiter is deformed into an oval shape during the impact event which leads to the gencration of large tensile stresses and thus damage near the sides of the steel cylinder. In these analyses, we have assumed that the limiter is foamed in place and that the foam remains bonded to the steel cylinder. The analyses with the coupled theory suggests that debonding or foam failure will occur near the sides of the steel cylinder. 
Table 8.1. Material Parameters Ised in Finite Element Analyses.

\begin{tabular}{|c|c|}
\hline Alıminumm: & $\begin{array}{l}\text { Young's Modulus }=10.0 \mathrm{E}+06 \mathrm{psi} \\
\text { Poisson's Ratio }=0.30 \\
\text { Density }=2.5 \mathrm{E}-041 \mathrm{~h} \mathrm{~s} \mathrm{~s}^{2} / \mathrm{in}^{4}\end{array}$ \\
\hline Sitecel: & $\begin{array}{l}\text { Young's Modulus }=29.0 \mathrm{E}+06 \mathrm{psi} \\
\text { Poisson's Ratio }=0.30 \\
\text { Density }=7.0 \mathrm{E}-04 \mathrm{lb} \mathrm{s}^{2} / \mathrm{in}^{4}\end{array}$ \\
\hline Foam: & $\begin{array}{l}\text { (von Mises Plasticity Model) } \\
\text { Young's Modulus }=3000.0 \mathrm{psi} \\
\text { Poisson's Ratio }=0.0 \\
\text { Density }=7.5 \mathrm{E}-06 \mathrm{Ib} \mathrm{s}^{2} / \mathrm{in}^{4} \\
\text { Yield Strength }=110.0 \mathrm{psi} \\
\text { Hardening Modulus }=0.0 \mathrm{psi}\end{array}$ \\
\hline Foam: & 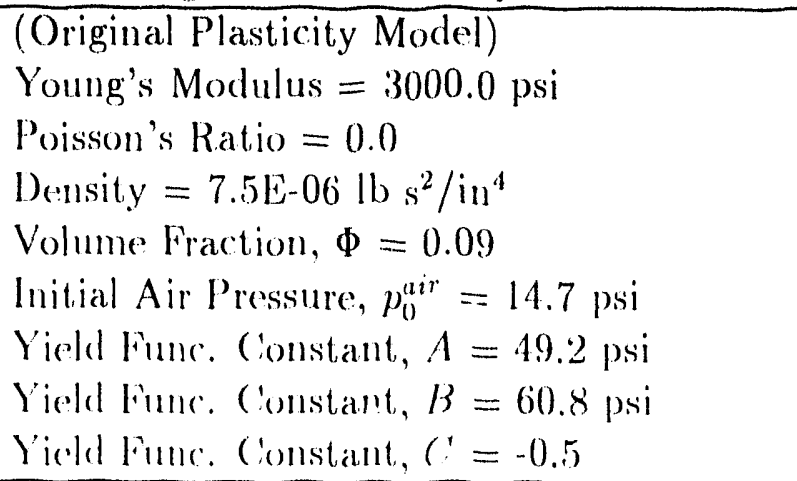 \\
\hline Foamll: & 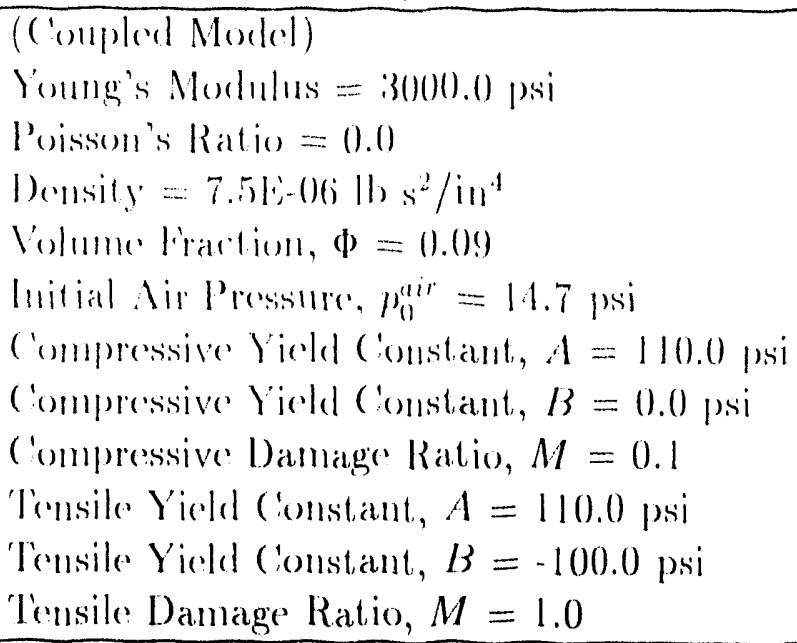 \\
\hline
\end{tabular}




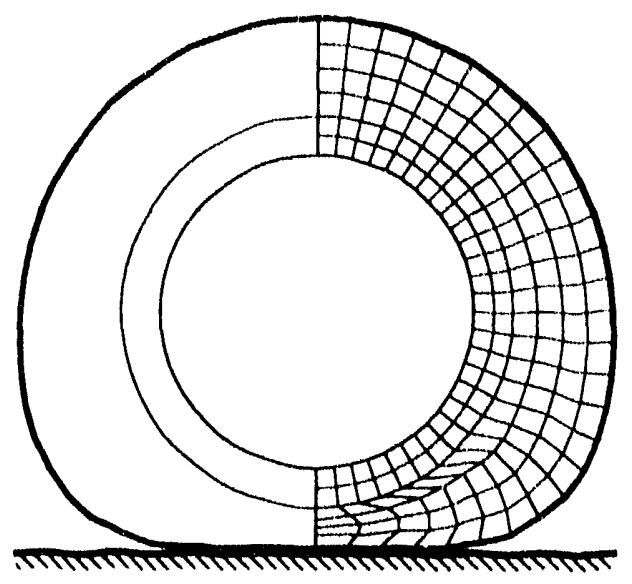

(a) conventional deviatoric plasticity

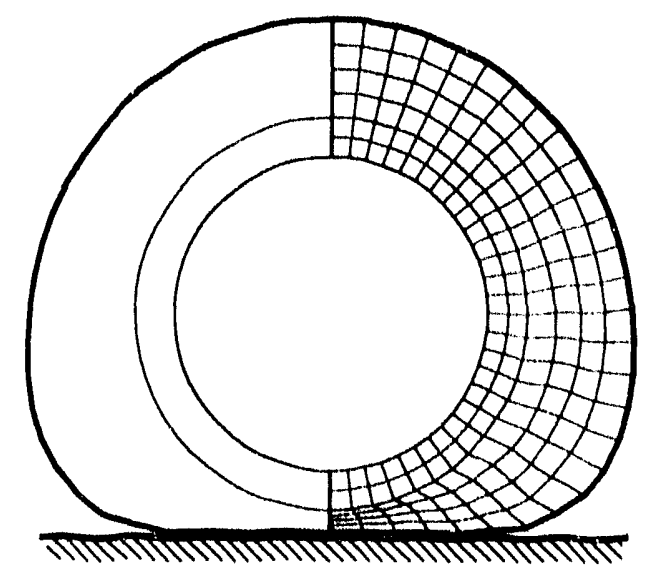

(b) original foam plastiticy

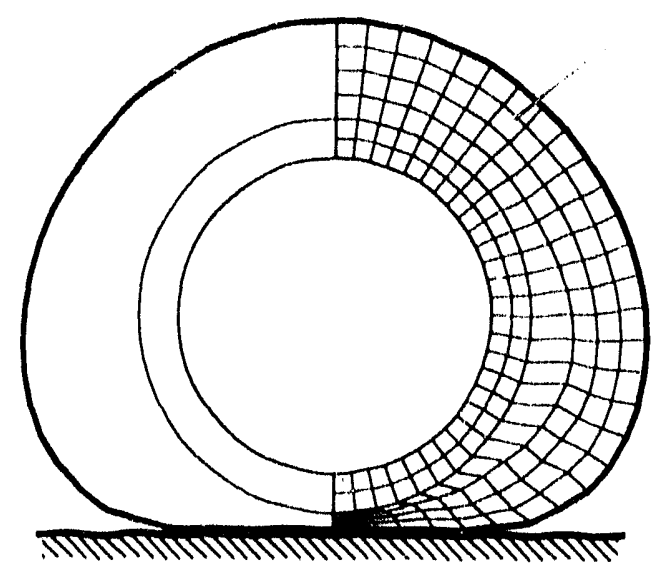

(c) rontimmum damage compled with plasticity

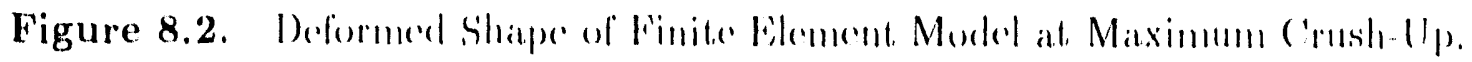




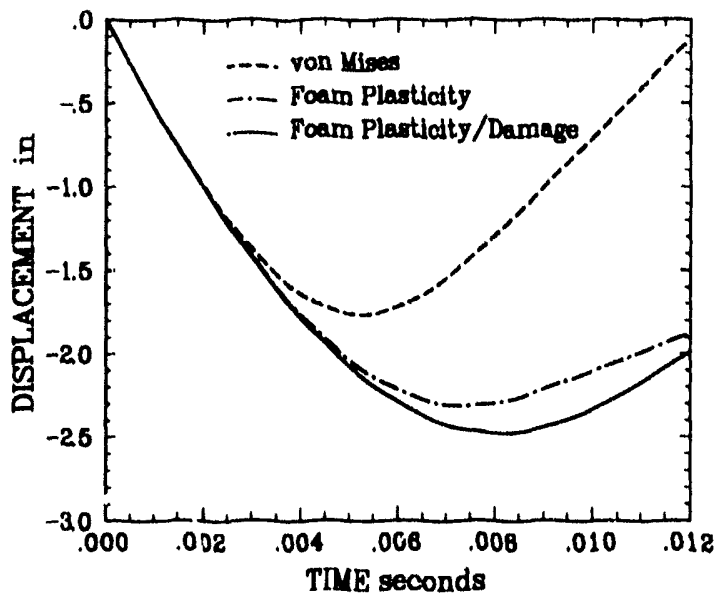

Figure 8.3. Dinplacement of Steel Cylinder Predicted by the Analyses.

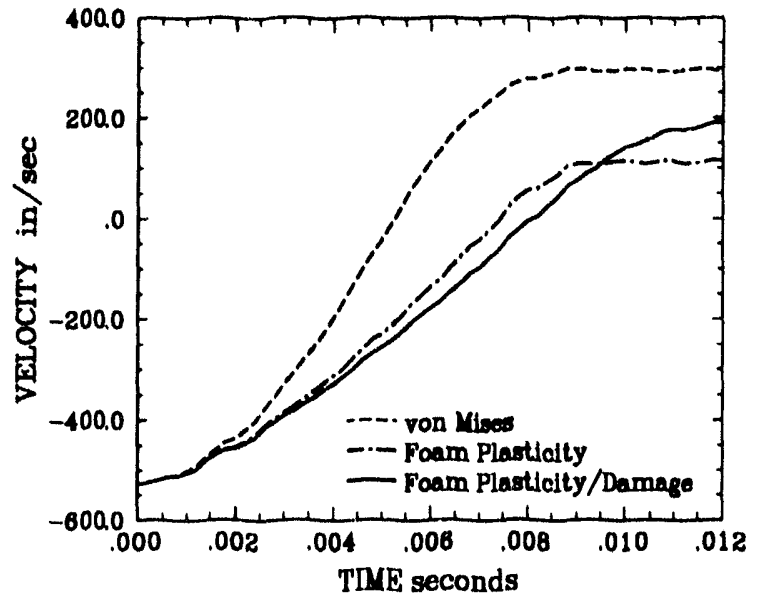

Figure 8.4. Velocity of Steel ('ylinder Predicted by the Analyses.

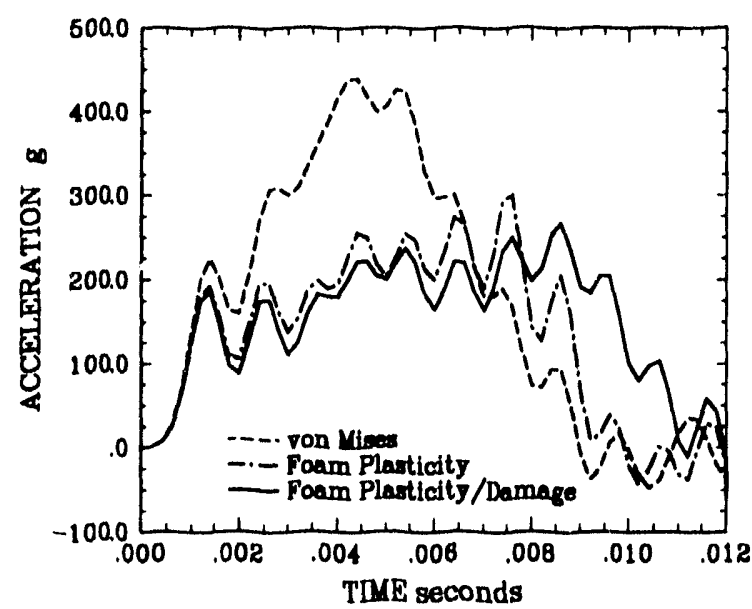

Figure 8.5. Acceleration of Steel Cylinder Predicted by the Aualyses. 



\section{CONCLUSIONS AND FUTURE WORK}

The primary focus of this rescarch was the development of constitutive theories for cellular materials that not only eapture the elastic response of these materials hut also the plastic deformation, damage and localized deformation that is generated when these materials are subjected to crushing loads. The constitutive relations developed for these materials use a combination of plasticity and continuum damage theories. Plasticity is a constitutive theory with a well-developed structure. Continumm damage theories, on the other hand, are just currently being developed (Krajcinovic, 1989). In this paper, a rather general continum damage theory with a structure similar to existing plasticity theories is developed. Ise of a similar structure simplifies the development of coupled plasticity-continum damage theories which share common yield (damage) surfaces. In this report, a coupled theory was developed for rigid polyurethane foam.

When cellular solids are loaded into the inelastic regine, they often exhibit deformations that are localized into small regions at some point in the loading process. Localization is often associated with satisfaction of the classical discontinuous bifurcation criterion. Here we propose that the loss of strong ellipticity condition should be used in place of the rassical discontinnous bifureation criterion as a necessary condition for localization. The application of the strong ellipticity criterion implies that a bifurcation mode associated with loss of positive definteness of the symmetric part of the aconstic tensor must he identified rather than a mole associated with the lirst zero eigenvalue of the acoustie tensor itsell. The eigensystem for the symmetric part of the tangent stiffness tensor is obtained for several different plasticity and continumm damage models. This cigensystem provides information ahout hoth diffuse and discontimuons bilureations. Matcrial properties, boundary ronditions, and body geometry are all shown to affect the diffuse and loralized doformation modes that are generated. Numerons experimental observations of necking and localization in motal speremens subject to varions hommdary conditions are explained with a hilureation analysis of a ven Mises plasticity model. Bifuration analyses were then performed on potential constitutive theories for collular solids. $A$ comparison of results from the bifurealion analyses with experimental olservations of localized deformations helpeed in the identification of appropriate constitutive theories for rollular materials.

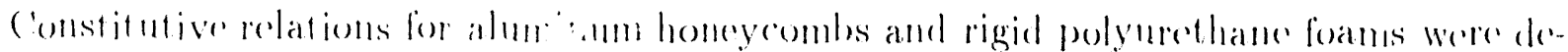
voloped and implemented in both statio and dynamic finite clement conles. $A$ mmonher of static tests on rigid polyurethane foam were analyzed and the results from the finite clement analyses were compared with experimental observations. Finally a typical impact problem was analyzed using various constitutive models for the polyurethane foam to demonstrate the effects of using varions constitutive descriptions for the foam. 
Cellular solids exhibit some additional features that are not captured by the constitutive theories which were developed in this work. First, dynamic tests on cellular solids indicate. that the strength of these materials is strain-rate dependent. The models developed in this paper could be modified to capture this rate dependence. For example, the yield function for the skeleton part of the models could be modified to include the strain-rate dependence. Next, the cyclic tests on this material indicated that a hysteresis loop is generated by unloading and reloading in the elastic regime. For the models developed in this paper, the elastic response is assumed to be linear. The hysteresis exhibited in the experiments may be due to the behavior of the polyurethane cell wall material or to the escape and reentry of the air from the sample during the cyclic loading. The motion of the fluid would have to be modeled and coupled with the skeleton model to capture the effect of fluid flow. The coupling between the fluid flow calculation and the structural calculation could be rather complex. For example, a large pressure gradient could cause damage and the amount of damage generated by crushing or fluid flow would have an effect on the rate of fluid flow. The solution of this coupled problem was beyond the scope of the current work. Finally, it is clear that a significant amount of work is still needed in the area of developing robust numerical procedures before it will be possible to routinely solve localization problems.

The response of cellular materials is complex with several modes of localized deformation exhibited when stress paths are allowed to span a large range. One aspect of the verification of a constitutive model is to show that the predicted orientation and nature of these localized deformation modes agree with experiments. Because of the lack of a routine procedure for theoretically obtaining such modes, this type of verification is practically never done for any material model, let alone those developed for cellular materials. The study of bifurcation phenomena presented here represents a significant enhancement to current technology, and provides an additional important mechanism for evaluating constitutive theories. 


\section{REFERENCES}

Aronofsky, J. (1951), The Formation of a Necked Region and Fracture Along an Oblique: Line in Flat Tensile Bars, Proc. 1st U.S. National Congress of Applied Mechanics, ASME Press, New York, 521-532.

Bazant, Z.P., and Lin, F. (1988), Nonlocal Smeared Cracking Model for Concrete Fracture, J. Struct. Engr. 114, 2493-2510.

Beaver, P.W. (1983), Localized Thinning, Fracture and Formability of Aluminium Shect Alloys in Biaxial Tension, J. Mech. Work. Technol. 7, 215-231.

Belytschko, T., Fish, J. and Engelmann, B.E. (1988), A Finite Element with Embedded Locaization Zones, Comp. Meth. Appl. Mech. Engr. 70, 59-89.

Benallal, A., Billardon, R. and Geymonat, G. (1989), Conditions de bifurcation a l'interieur ct aux frontieres pour une classe de materiaux non-standards, (.R. Acad. Sci. Paris 308, 89:3-898.

Benallal, A., Billardon, R. and Geymonat, (r. (1990), Phenomenes de localisation a la fronticre d'un solide, ('.R. Acad. S'ci. Paris 310, 679-684.

Biffle, J. H. (1989), JACSD - A Three-Dimensional Finite Element Computer Program for the Nonlinear Quasi-Static Response of Solids with the Conjugate Gradient Method, SAND87-1305, Sandia National Laboratories, Albuquerque, New Mexico.

Bigoni, D. and Hueckel, T. (1991), Uniqueness and Localization - Associative and NonAssociative Elastoplasticity, Int. J. Solids Struct. 28, 197-213.

Chen, Z., Neilsen, M.K., and Schreyer, H.L. (1991), A Secant Structural Solution S'cheme: for Incremental Damage and Plasticity, Proceedings of the 3rd Intl. Conf. on Constitutive Laws for Engineering Materials, Edited by Desai, C.S., Krempl, E., Frantziskonis, (i. and Saadatmanesh, H., ASME Press, New York, 677-682.

Coleman, B.D. and (Gurtin, M. (1967), Thermodynamies with Internal State Variables, J. Chem. Phys. 47, 597-613.

Crisfield, M.A. (1981), A Fast Incremental/Iterative Solution Procedure that Handles S'nap-Through, ('omput. Struct. 13, 5.5-62.

de Borst, R. (1986), Nonlinear Analysis of Frictional Materials, Ph. D. Dissertiation, Delft, University of Technology, Delft, The Netherlands. 
Donald, S. and Maji, A. (1992), Static Testing of Aluminum Honeycomb and Polyurethane Foams, contractors report to Sandia National Laboratories, Albuquerque, New Mexico, in press.

Doyle, E. N. (1971), The Development and Use of Polyurethane Products, Mc(iraw-Hill Book ('o., New York, 256-263.

Drucker, D.(. (1950), Some Implications of Work Hardening and Ideal Plasticity, Quart. Appl. Math. 7, 411-418.

Drucker, D.C. (1959), A Definition of a Stable Inelastic Material, J. Appl. Mech. 26, 101-106.

Gibson, L.J. and Ashby, M.F. (1988), Cellular Solids - Structure and Properties, Pergamon Press, New York.

Hadamard, J. (1903), Lecons sur la Propagation des Ondes et les Equations de L'Hydrodynamique, Hermann, Paris.

Hill, R. (1958), A General Theory of Uniqueness and Stability in Elastic-Plastic Solids, J. Mech. Phys. Solids 6, 236-249.

Hill, R. (1962), Acceleration Waves in Solids, J. Mech. Phys. Solids 10, 1-6.

Hill, R. (1991), A Theoretical Perspective on In-plane Forming of Sheet Metal, J. Mech. Phys. Solids 39, 295-307.

Hutchinson, J.W., and Miles, J.P. (1974), Bifurcation Analysis and the Onset of Necking in an Elastic/Plastic Cylinder Under Uniaxial Tension, J. Mech. Phys. Solids 22, 61-71.

Kachanov, L.M. (1986), Introduction to Continuum Damage Mechanics, Martinus Nijhoff, Dordrecht, The Netherlands.

Klintworth, J.W. and Stronge, W.J. (1988), Elasto-Plastic Yifld Limits and Deformalion. laus for Transwersely ('rushed Honcycombs, Int. J. Mech. Sci. 30, 273-292.

Krajoinovir, D. (1989), Damage Mechanies, Mech. Mater. 8, 117-197.

Larsson, R. and Runesson, K. (1991), Finile Element Simulation of Localized Plastic Deformation, Arch. Appl. Mech. 61, 305-317.

Leroy, Y. and Ortiz, M. (1989), Finite Element Analysis of Strain Localization in Frictional Materials, Int. J. Numer. Anal. Meth. Geomech. 13, 53-74.

Malvern, L.E. (1969), Introduction to the Mechanics of a Continuous Medium, Prentice-Hall, Englewood Cliffs, New Jersey. 
Mandel, J. (1966), Conditions de Stabilite' et Postulat de: Drucker, Published in Rheologie et Mecanique des Sols, Symposium Grenoble, Edited by Kravtchenko, J. and Sirieys, P.M., Springer-Verlag, New York.

Miles, J.P. (1975), The Initiation of Necking in Rectangular Elastic/Plastic Specimens Under Uniaxial and Biaxial Tension, J. Mech. Phys. Solids 23, 197-213.

Nadai, A. (1950), Theory of Flow and Fracture of Solids, McGraw-Hill Book ('o., New York.

Needleman, A. (1972), A Numerical Study of Necking in Circular Cylindrical Bars, J. Mech. Phys. Solids 20, 111-127.

Needleman, A. and Tvergaard, V. (1984), Finite Element Analysis of Localization in Plasticity, Published in Finite Elements - Special Problems in Solid Mechanics, Edited by Oden, J.T. and Carey, C.F., Prentice-Hall, Englewood Cliffs, New Jersey.

Neilsen, M.K., Morgan, H.S., and Krieg, R.D. (1987), A Phenomenological Constitutive Model for Low Density Polyurethane. Foams, SAND86-2927, Sandia National Laboratories, Albuquerque, New Mexico.

Neilsen, M.K., (hen, Z., and Schreyer, H.L. (1991), A Structural Constitutive Algorithm Based on Continuum Damage Mechanics for Softening with Snap-Back, Proceedings of the 3rd Intl. Conf. on Constitutive Laws for Engineering Materials, Edited by Desai, (.$S$. , Krempl, E., Frantziskonis, C. and Saadatmanesh, H., ASME Press, New York, $751-754$.

Ortiz, M., Leroy, Y., and Needleman, A. (1987), A Finite Element Method for Localized Failure Analysis, (omp. Meth. Appl. Mech. Engr. 61, 189-214.

Ottosen, N.S., and Runesson, K. (1991), Properties of Discontinuous Bifurcation Solutions in Elasto-Plasticity, Int. J. Solids Struct. 27, 401-421.

Patel, N.R. and Finnie, I. (1969), The Deformation and Fracture of Rigid Cellular Plastics linder Multiarial Stress, Report U('RL-13420, Lawrence Livermore Laboratory, Livermore, ('alifornia.

Pietruszak, S'T' and Mroz, \%. (1981), Finite Elcment Analysis of Deformation of StrainSoftening Malerials, lut. J. Numer. Meth. Engr. 17, 327-334.

Raniecki, B. and Bruhus, O.T'. (1981), Bounds to Bifurcation Stresses in Solids with Non-Associated Plastic Flow Law at Finite Strain, J. Mech. Phys. Solids 29, 153-172.

Rice, J.R. (1976), The Localization of Plastic Deformation, Published in Theoretical and Applied Mechanics, 14th IUTAM Congress, Edited by Koiter, W.T., NorthHolland Pub. ('o., Amsterdam. 
Rice, J.R. and Rudnicki, J.W. (1980), A Note on Some Features of the Theory of Localization of Deformation, Int. J. Solids Struct. 16, 597-605.

Riks, E. (1979), An Incremental Approach to the Solution of Sinapping and Buckling Problems, Intl. J. Solids Struct. 15, 524-551.

Rudnicki, J.W. and Rice, J.R. (1975), Conditions for the Localization of Deformation in Pressure-Sensitive Dilatant Materials, J. Mech. Phys. Solids 23, 371-394.

Runesson, K., Ottosen, N.S., and Peric, D. (1991), Discontinuous Bifurcations of ElasticPlastic Solutions at Plane Stress and Plane Strain, lntl. .J. Plast. 7, 99-121.

Schreyer, H.L. and ('hen, Z. (1986), Onc-Dimensional Softening with Localization, .J. Appl. Mech. 53, 791-797.

Schreyer, H.L. (1990a), Inelastic Constitutive Theorics, Department of Mechanical Engineering ('lass Notes, Iniversity of New Mexico, Albuquerque, New Mexico.

Schreyer, H.L. (1990b), Analytical Solutions for Nonlinear Strain-Ciradient S'oftening and Localization, J. Appl. Mech. 57, 522-527.

Shaw, M.(: and Sata, T. (1966), The Plastic Behavior of Cellular Malcrials, Int. J. Mech. Sici. 8, 469-478.

Simo, J.('. and Hughes, T.J.R. (1986), Elastoplasticity and Viscoplasticity - Computational Aspects, monograph.

Stone, (. M. (1992), SANT'(OS' - A Two-Dimensional Finite Element Program for the Quasistatic, Large Deformation, Inclastic Response of Solids, SAND90-0543, Sandia National Laboratories, Albuquerque, New Mexico.

Storen, S. and Rice, J.R. (1975), Localized Necking in Thin Sheets, J. Mech. Phys. Solids 23, $421-441$.

Taylor, L. M. and Flanagan, D. P. (1986), A Two-Dimensional Transient Solid Dynamics Program, SAND86-0594, Sandia National Laboratories, Albuquerque, New Mexico.

Thomas, T.Y. (1961), Plastic Flow and Fracture in Solids, Academic Press, New York.

Triantafillon, T'.(:, and (iibson, L.J. (1990), Constitutive Modelling of Elastic'l'lastic Open-Cell Foams, J. Engr. Mech. 116, 2772-2778.

Valanis, K.C.(1989), Banding and Stability in Plastic Matcrials, Acta Mech. 79, 113-141.

Vardoulakis, I., Cioldscheider, M., and Gudehus, (1. (1978), Formation of Shear Bands in. Sand Bodies as a Bifurcation Problem, Int. J. Numer. Anal. Metl. Cieomech. 2, 99-128. 
Warren, W.E., Kraynik, A.M. (1987), Foam Mechanics: The Linear Elastic Response of Two-Dimensional S'patially Periodic Celluiar Materials, Mech. Mater. 6, 27-37.

Warren, W.E., Kraynik, A.M., and Stone, (.M. (1989), A Constitutive Model for TwoDimensional Nonlinear Elastic Foams, J. Mech. Phys. Solids 37, 717-733.

Zaslawsky, M. (1973), Multiaxial Stress Studies on Rigid Polyurethane Foam, Exper. Mech. 13, 70-76. 


\section{Unlimited Release}

Prof. S. Karnawat

Turtle Mountain College

Box 340

Belcourt, ND $58316^{\circ}$

Prof. R.D. Krieg

Engineering Science and Mechanics

301 Perkins Hall

University of Tennessee

Knoxville, TN 37923-6819

M.W. Lewis, MS J-576

Los Alamos National Lab

P.O. Box 1663

Los Alamos, NM 87545

Prof. A.K. Maji

Dept. of Civil Engineering

University of New Mexico

Albuquerque, NM 87131

Prof. H.L. Schreyer (3)

Dept. of Mechanical Engineering

University of New Mexico

Albuquerque, NM 87131

Prof. S. Steinberg

Dept. of Mathematics and Statistics

University of New Mexico

Albuquerque, NM 87131

Prof. D.Y'. T'zou

Dept. of Mechanical Engineering

University of New Mexico

Albuquerque, NM 87131

Prof. W.E. Warren

Dept. of Civil Engineering

Texas A \& M University

College Station, TX 77843-3136

Prof. F. Yazdani

Department of Civil Engineering

North Dakota State University

Fargo, ND 58105

Prof. H.M. Zbib

Dept. of Mechanical and Matls. Engineering

Washington State University

Pullman, WA 99164-2920
Internal Distribution:

1000 P.A. Fleury

1425 J.H. Biffle

1425 S.W. Attaway

1432 H.E. Fang

1500 D.J. McCloskey

1501 C.W. Peterson

Route to: $1512,1513,1551,1552$

1502 P.J. Hommert

Route to: $1503,1511,1553,1554$

1512 A.M. Kraynik

1561 H.S. Morgan

1561 M.K. Neilsen (25)

1561 M.W. Heinstein

1561 E.L. Hoffman

1561 ( .M. Stone

1561 J.R. Weatherby

1561 C.W. Wellman

1562 R.K. Thomas

1562 F.J. Mello

1562 (i.I). Sjaardema

1562 A.M. Slavin

5165 N.R. Hansen

5165 A.R. York

(6642 J.D. Pierce

6642 D.C. Harding

8700 R.C. Wayne

8702 (. W. Robinson

87.41 C.A. Benedetti

8741 M.L. Chiesa

8742 P.E. Nielan

8743 M.L. Callabresi

8743 D.J. Bammann

8746 W.A. Kawahara

7141 Technical Library (5)

7151 Technical Publications

7613-2 Document Processing for DOE/OSTI (15)

$8523 \cdot 2$ Central Technical Files 

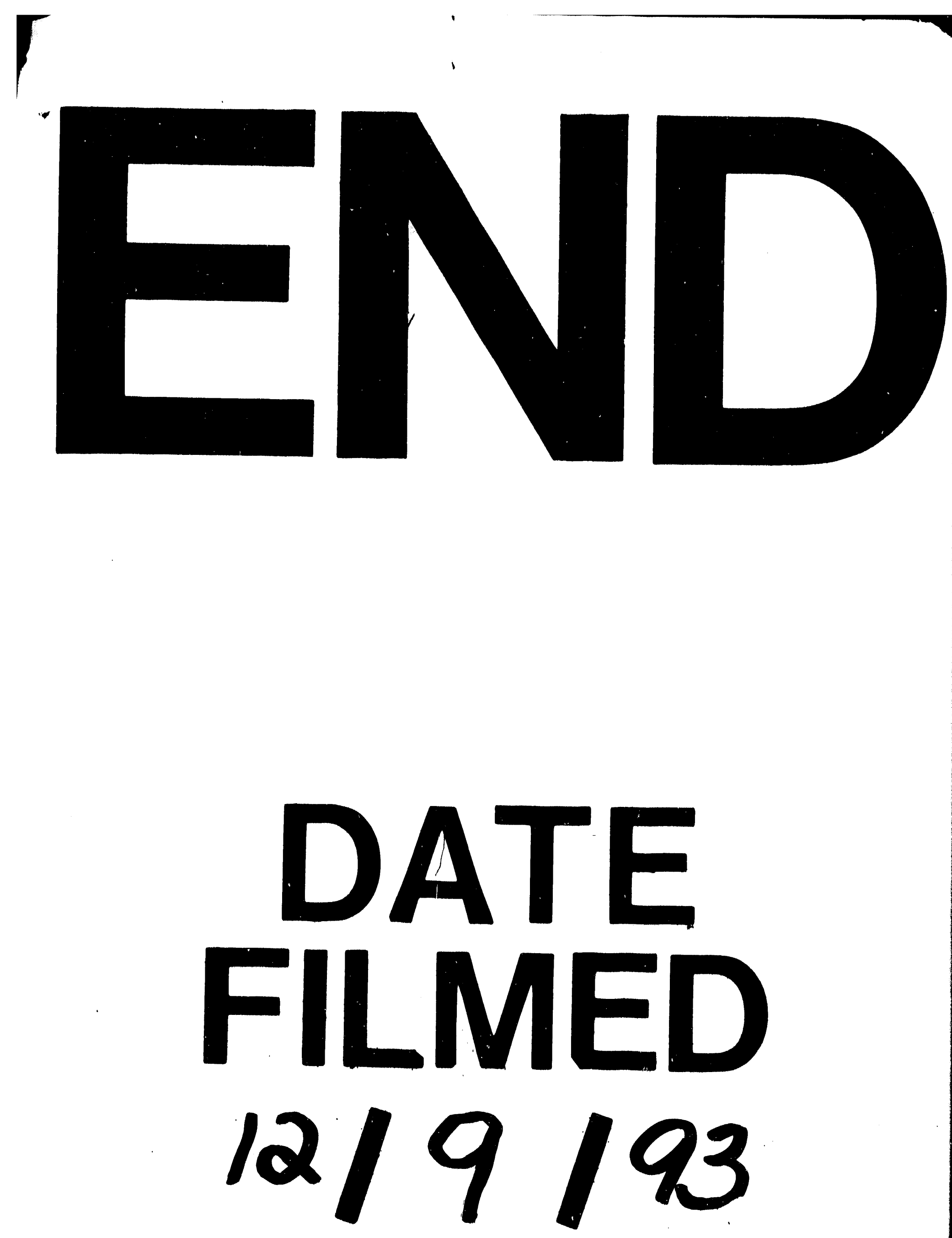
